\title{
RIESZ CONTINUITY OF THE ATIYAH-SINGER DIRAC OPERATOR UNDER PERTURBATIONS OF THE METRIC
}

\author{
LASHI BANDARA, ALAN MCINTOSH, AND ANDREAS ROSÉN
}

\begin{abstract}
We prove that the Atiyah-Singer Dirac operator $\not_{\mathrm{g}}$ in $\mathrm{L}^{2}$ depends Riesz continuously on $\mathrm{L}^{\infty}$ perturbations of complete metrics g on a smooth manifold. The Lipschitz bound for the map $\mathrm{g} \rightarrow \not_{\mathrm{g}}\left(1+\not_{\mathrm{g}}^{2}\right)^{-\frac{1}{2}}$ depends on bounds on Ricci curvature and its first derivatives as well as a lower bound on injectivity radius. Our proof uses harmonic analysis techniques related to Calderón's first commutator and the Kato square root problem. We also show perturbation results for more general functions of general Dirac-type operators on vector bundles.
\end{abstract}

\section{Contents}

1. Introduction

Acknowledgements

2. Setup and the statement of the main theorem

3. Applications to the Ativah-Singer Dirac operator 10

4. Reduction to quadratic estimates 25

5. Quadratic estimates 33

$\begin{array}{ll}\text { References } & 47\end{array}$

\section{INTRODUCTION}

In this paper we prove perturbation estimates for self-adjoint first-order partial differential operators $\mathrm{D}$ and $\tilde{\mathrm{D}}$ of Dirac type, elliptic with domains $\mathrm{W}^{1,2}(\mathcal{M}, \mathcal{V})$ in $\mathrm{L}^{2}(\mathcal{M}, \mathcal{V})$, on vector bundles $\mathcal{V}$ over complete Riemannian manifolds $(\mathcal{M}, \mathrm{g})$. A typical quantity to bound is

$$
\left\|\frac{\tilde{\mathrm{D}}}{\sqrt{I+\tilde{\mathrm{D}}^{2}}}-\frac{\mathrm{D}}{\sqrt{\mathrm{I}+\mathrm{D}^{2}}}\right\|_{\mathrm{L}^{2}(\mathcal{M}, \mathcal{V}) \rightarrow \mathrm{L}^{2}(\mathcal{M}, \mathcal{V})} .
$$

Our motivating and main example is when $\mathrm{D}=\not D$ is the Atiyah-Singer Dirac operator $\not D$ on $\mathcal{M}$, acting on sections of a given spin bundle $\mathcal{V}=\not \Delta \mathcal{M}$ over $(\mathcal{M}, \mathrm{g})$. The perturbations $\tilde{\mathrm{D}}$ we consider arise from the pullback of the Atiyah-Singer operator on a nearby manifold $(\mathcal{N}, \mathrm{h})$. More precisely, we have a diffeomorphism $\zeta: \mathcal{M} \rightarrow \mathcal{N}$ which induce a map $\forall: \Delta \mathcal{M} \rightarrow \Delta \mathcal{N}$ between the two spinor bundles, and we set $\tilde{\mathrm{D}}:=\bigcup^{-1} \not D_{\mathcal{N}} \forall$ on $\mathcal{M}$. For the construction of the induced spinor pullback, we follow [8] by Bourguignon and Gauduchon and build this from the isometric factor of the polar factorisation of the differential of $\zeta$.

Date: July 4, 2018.

2010 Mathematics Subject Classification. 58J05, 58J37, 58J30, 35J46, $42 \mathrm{~B} 37$.

Key words and phrases. Riesz continuity, Dirac operator, spectral flow, Kato square root problem, functional calculus. 
The perturbation (1.1) is for the symbol $f(\lambda)=\lambda / \sqrt{1+\lambda^{2}}$ in the functional calculi of the operators $\mathrm{D}$ and $\tilde{\mathrm{D}}$. This will yield continuity results in the Riesz metric given by (1.1) for unbounded self-adjoint operators. However, our method of proof applies equally well to any other symbol $f(\lambda)$ which is holomorphic and bounded on the neighbourhood $\mathrm{S}_{\omega, \sigma}^{\circ}:=\left\{x+i y: y^{2}<\tan ^{2} \omega x^{2}+\sigma^{2}\right\}$ of $\mathbb{R}$ for some $0<\omega<\pi / 2$ and $\sigma>0$. Our Riesz continuity result is non-trivial as it entails cutting through the spectrum at infinity with the added complication that the symbol has different limits at infinity $\left(\lim _{\lambda \rightarrow \pm \infty} f(\lambda)= \pm 1\right)$. This should be compared to the weaker continuity result for the graph metric

$$
\left\|\frac{\tilde{D}-\mathrm{i}}{\tilde{\mathrm{D}}+\mathrm{i}}-\frac{\mathrm{D}-\mathrm{i}}{\mathrm{D}+\mathrm{i}}\right\|_{\mathrm{L}^{2}(\mathcal{M}, \mathcal{V}) \rightarrow \mathrm{L}^{2}(\mathcal{M}, \mathcal{V})}
$$

for unbounded self-adjoint operators, which is simpler since the symbol $g(\lambda)=$ $(\lambda-\mathrm{i}) /(\lambda+\mathrm{i})$ is holomorphic at $\infty$.

The Riesz and graph topologies are of great importance in the study of self-adjoint unbounded operators because of their connection to the spectral flow. Loosely speaking, this is the net number of eigenvalues crossing zero along a curve from the unit interval to the set of self-adjoint operators. The study of the spectral flow was initiated by Atiyah and Singer in [2] since it has important connections to particle physics. Their focus, however, was on bounded Fredholm self-adjoint operators and their point of view was largely topological. An analytic formulation of the spectral flow also exists due to Phillips in [25].

In the bounded case, the choice of topology for the study of the spectral flow is canonically given by the norm topology. However, in order to study differential operators, the unbounded case needs to be considered. Here, a choice of topology needs to be made and the graph metric is most commonly used in the study of the spectral flow, primarily since it is easier to establish continuity in this topology. However, the Riesz topology is a preferred alternative since it better connects to topological and $K$-theoretic aspects of the spectral flow that were observed in [2] for bounded operators. Further details of the relation between different metrics on the set of unbounded self-adjoint operators can be found in [20] by Lesch. Moreover, the survey paper [7] by Booß-Bavnbek provides a recent account of problems remaining in field of spectral flow.

Since in this paper we establish results in the Riesz topology, of particular relevance is Proposition 2.2 in [20] where it is proved that

$$
\left\|\frac{\tilde{\mathrm{D}}}{\sqrt{\mathrm{I}+\tilde{\mathrm{D}}^{2}}}-\frac{\mathrm{D}}{\sqrt{\mathrm{I}+\mathrm{D}^{2}}}\right\|_{\mathrm{L}^{2}(\mathcal{M}, \mathcal{V}) \rightarrow \mathrm{L}^{2}(\mathcal{M}, \mathcal{V})} \lesssim\|\tilde{\mathrm{D}}-\mathrm{D}\|_{\mathrm{W}^{1,2}(\mathcal{M}, \mathcal{V}) \rightarrow \mathrm{L}^{2}(\mathcal{M}, \mathcal{V})}
$$

holds for small perturbations $\tilde{D}$ of $\mathrm{D}$ with both operators self-adjoint and with domain $\mathrm{W}^{1,2}(\mathcal{M}, \mathcal{V})$. We achieve a non-trivial strengthening of this estimate for Dirac-type differential operators, using techniques from harmonic analysis. The structure of the perturbation that we consider is

$$
\tilde{\mathrm{D}}-\mathrm{D}=A_{1} \nabla+\operatorname{div} A_{2}+A_{3}
$$


where $A_{1}, A_{2}$ and $A_{3}$ are bounded multiplication operators $\mathrm{T}^{*} \mathcal{M} \otimes \mathcal{V} \rightarrow \mathcal{V}, \mathcal{V} \rightarrow$ $\mathrm{T}^{*} \mathcal{M} \otimes \mathcal{V}$ and $\mathcal{V} \rightarrow \mathcal{V}$ respectively. Typically in applications, and in particular for the Atiyah-Singer Dirac operator, one can achieve

$$
\left\|A_{i}\right\|_{\infty} \lesssim\|\tilde{\mathrm{g}}-\mathrm{g}\|_{\infty}
$$

where $\mathrm{g}$ is the metric on $\mathcal{M}$ and $\tilde{\mathrm{g}}=\zeta^{*} \mathrm{~h}$ is the metric on $\mathcal{N}$ pulled back to $\mathcal{M}$. In order to conclude small Riesz distance between D and $\tilde{D}$ using the aforementioned Proposition 2.2 in [20], one would need not only smallness of $\left\|A_{i}\right\|_{\infty}$ but also smallness of $\left\|\nabla^{\mathrm{g}} A_{2}\right\|$. Via our methods, we are able to dispense this requirement and only require the finiteness of $\left\|\nabla^{\mathrm{g}} A_{2}\right\|$.

In Theorem 2.4, which is our main result, we prove the perturbation estimate

$$
\|f(\tilde{\mathrm{D}})-f(\mathrm{D})\| \lesssim \max _{i}\left\|A_{i}\right\|_{\infty}\|f\|_{\mathrm{Hol}^{\infty}\left(\mathrm{S}_{\omega, \sigma}^{\mathrm{o}}\right)},
$$

where the implicit constant depends on the geometry of $\mathcal{V} \rightarrow \mathcal{M}$ and the operators $\mathrm{D}$ and $\tilde{\mathrm{D}}$ as described in the hypothesis (A1)-(A9) preceding Theorem 2.4. In Theorem 3.1, we specialise Theorem 2.4 to the case where the operators D and $\tilde{D}$ are the Atiyah-Singer Dirac operators as previously discussed. Here, the implicit constant depends roughly on the $\mathrm{C}^{0,1}$ norm of $\tilde{g}$ and $\mathrm{C}^{2}$ norm of $\mathrm{g}$. Injectivity radius bounds coupled with bounds on Ricci curvature and its first derivatives allow us to obtain uniformly sized balls corresponding to harmonic coordinates at every point. Moreover, we obtain uniform $\mathrm{C}^{2}$ control of the metric $\mathrm{g}$ in each such chart. Therefore our result, unlike Proposition 2.2 in [20], will apply to metric perturbations with $\tilde{g}-g$ small only in $\mathrm{L}^{\infty}$ norm, under uniform $\mathrm{C}^{2}$ control of $\mathrm{g}$ and uniform $\mathrm{C}^{0,1}$ control of $\tilde{g}$ in each such chart. A concrete example of such metrics are $\mathrm{g}=\mathrm{I}$ and $\tilde{\mathrm{g}}(x)=(1+\varepsilon \sin (|x| / \varepsilon)) \mathrm{I}$ on $\mathbb{R}^{n}$.

The main work in establishing (1.3) is to prove quadratic estimates of the form

$$
\int_{0}^{1}\left\|\frac{t \tilde{\mathrm{D}}}{\mathrm{I}+t^{2} \tilde{\mathrm{D}}^{2}} B \frac{\mathrm{I}}{\mathrm{I}+t^{2} \mathrm{D}^{2}} u\right\|_{\mathrm{L}^{2}(\mathcal{M}, \mathcal{V})}^{2} \frac{d t}{t} \lesssim\|B\|_{\mathrm{L}^{\infty}(\mathcal{M}, \mathcal{V})}^{2}\|u\|_{\mathrm{L}^{2}(\mathcal{M}, \mathcal{V})}^{2},
$$

where $B$ is a bounded operator, a multiplication operator, or special kind of a singular integral. The use of such quadratic estimates to bound functional calculi goes back to the work of Coifman, McIntosh and Meyer [13, 14] on Calderón's problem on the boundedness of the Cauchy integral of Lipschitz curves. The quadratic estimates that we require in this paper are at the level of those needed to bound Calderón's first commutator. An additional technical difficulty for us in the present work is that $B$ also may involve a certain singular integral operator. To overcome this problem, we need a Riesz-Weitzenböck condition stated as hypothesis (A9).

The starting point for our work in this paper, was a twin result for the Hodge-Dirac operator $\mathrm{d}+\mathrm{d}^{*}$ proved by the last two named authors jointly with Keith in [4]. There it was proved, in the case of compact manifolds, that

$$
\left\|\operatorname{sgn}\left(\frac{\mathrm{d}_{\tilde{\mathrm{g}}}+\mathrm{d}_{\tilde{\mathrm{g}}}^{*}}{\sqrt{1+\left(\mathrm{d}_{\tilde{\mathrm{g}}}+\mathrm{d}_{\tilde{\mathrm{g}}}^{*}\right)^{2}}}\right)-\operatorname{sgn}\left(\frac{\mathrm{d}_{\mathrm{g}}+\mathrm{d}_{\mathrm{g}}^{*}}{\sqrt{1+\left(\mathrm{d}_{\mathrm{g}}+\mathrm{d}_{\mathrm{g}}^{*}\right)^{2}}}\right)\right\| \lesssim\|f\|_{\operatorname{Hol}_{(\infty, \sigma}^{\infty}\left(\mathrm{S}_{\omega, \sigma}^{\circ}\right)}\|\tilde{\mathrm{g}}-\mathrm{g}\|_{\infty},
$$


This made use not only of the methods from [14] described above, but also of stopping time arguments for Carleson measures from the solution of the Kato square root problem by Auscher, Hofmann, Lacey, McIntosh and Tchamitchian [3]. These techniques give results for perturbations when the domains of the Hodge-Dirac operators change, that is when no Lipschitz control of the metric is assumed, and even give holomorphic dependence of $\operatorname{sgn}\left(\mathrm{d}_{\mathrm{g}}+\mathrm{d}_{\mathrm{g}}^{*}\right)$ on the metric $\mathrm{g}$ and not only Lipschitz dependence. However, there are also reasons to prefer the softer methods used in this paper and to avoid the stopping time arguments. Namely, even though they make the implicit constant in (1.5) independent of any Lipschitz control of the metrics, this constant in applications may become too large for the estimate to be useful. Our plan is to return to the perturbation problem for the Hodge-Dirac operator in a forthcoming paper.

As aforementioned, since the Riesz topology is one of the most important operator topologies for unbounded self-adjoint operators, it is a natural question how much of the above estimates hold for more general Dirac type operators, and in particular the most fundamental Atiyah-Singer Dirac operator. For these operators we no longer have access to Hodge splittings, and it is not even clear that the Dirac operators exist as closed and densely-defined operators for rough metrics (measurable coefficients but locally bounded below). Therefore the perturbation estimates that we achieve in this paper, with the constant depending on the Lipschitz norm of the metrics, may be quite sharp. We do not even know however if it is possible to go beyond Lipschitz metrics for Dirac operators like the Atiyah-Singer one. In any case, as Lesch rightly points out in [20], it is more difficult to prove Riesz continuity as compared to other operator topologies and therefore, our results should have interesting applications to the study of spectral flow and to index theory of Dirac operators. Moreover, given the generality of Theorem 2.4, we anticipate that these applications will go beyond the fundamental case of the Atiyah-Singer Dirac operator that we consider as an application here.

The outline of this paper is as follows. Our main perturbation theorem, Theorem 2.4 for general Dirac-type operators is formulated in $\S 2.4$. Before stating it, we discuss the geometric and operator theoretic assumptions and we list quantities that the implicit constant in the estimate (1.3) depends on as hypotheses (A1)-(A9).

For the proof of Theorem 2.4, the reader may jump directly to $\S 4$ and $\S 5$. Independent of this, we first devote $\S 3$ to prove Theorem 3.1, which is an application of Theorem 2.4 to the Atiyah-Singer Dirac operator. For the sake of concreteness, we only consider this Dirac operator obtained from the standard spin representation of dimension $2^{\left\lfloor\frac{n}{2}\right\rfloor}$ and a given Spin structure. But we expect Theorem 3.1 to hold for more general Dirac-type operators on Dirac-bundles under similar geometric assumptions. The proof of Theorem 3.1 amounts to verifying (A1)-(A9) and the perturbation structure (1.2). A key observation regarding the latter is the following exploited in $\S 3.3$. A perturbation term $A_{3}$ of the form $A_{3}=\partial B$ (with $\partial$ denoting a partial derivative) with $\|B\|_{\infty}$ small, but with $\|\partial B\|_{\infty}$ only bounded, can be handed as terms $A_{1} \partial+\partial A_{2}$, with $B=A_{2}=-A_{1}$, since by the product rule, $(\partial B) f=\partial(B f)-B(\partial f)$. 
The proof of Theorem 2.4 in $\S 4$ and $\S 5$, brought together in $\S 5.6$, contains the following steps. Using the functional calculus of $\mathrm{D}$ and $\tilde{\mathrm{D}}$, the estimate of $\|f(\mathrm{D})-f(\tilde{\mathrm{D}})\|$ is reduced to the quadratic estimate (1.4) in Proposition 4.5 and 4.6. This quadratic estimate is obtained in three steps described by the formula (5.11), following a well known harmonic analysis technique used in the solution of the Kato square root problem with its origins from R. Coifman and Y. Meyer. For us, the last term $\gamma_{t} \mathbb{E}_{t} S f$ is not the main one, since the needed Carleson measure estimate follows directly from the self-adjointness of $\tilde{D}$, as shown in $§ 5.5$. The main term in (5.11) is rather the first, which localises the operator $\mathbf{Q}_{t}$, which is local on scale $t$, to the multiplication operator $\gamma_{t}$. Our problem here is the presence of $S=\nabla(\mathrm{iI}+\mathrm{D})^{-1}$, which is essentially a singular integral operator. To handle the non-local operator $S$ in Proposition 5.4, we require some smoothness of $\mathrm{D}$, guaranteed by the Riesz-Weitzenböck condition (2.5). In [9], Bunke obtains such an estimate, but with assumptions on the Riemannian curvature tensor in place of the Ricci curvature. Our proof here is inspired by the improvements that Hebey presents using harmonic coordinate charts under the presence of positive injectivity radius and bounds on Ricci curvature to prove density theorems for Sobolev spaces of functions on noncompact manifolds in [17].

\section{ACKNOWLEDGEMENTS}

The first author was supported by the Knut and Alice Wallenberg foundation, KAW 2013.0322 postdoctoral program in Mathematics for researchers from outside Sweden. The second author appreciates the support of the Mathematical Sciences Institute at The Australian National University, and also the support of Chalmers University of Technology and University of Gothenburg during his visits to Gothenburg. Further he acknowledges support from the Australian Research Council. The third author was supported by Grant 621-2011-3744 from the Swedish Research Council, VR.

We would like to thank Alan Carey and Krzysztof P. Wojciechowski for discussions about the relevance of our approach to open questions involving spectral flow for paths of unbounded self-adjoint operators. Unfortunately Wojciechowski became ill and died before these promising early discussions could be developed.

\section{Setup And the statement of the main theorem}

2.1. Notation. Throughout this paper, we assume Einstein summation convention and use the analysts inequality $a \lesssim b$ to mean that $a \leq C b$, where $C>0$, and equivalence $a \simeq b$. The characteristic function on a set $E$ will be denoted by $\chi_{E}$. Throughout, we will identify vectorfields and derivations. That is, for a function $f$ differentiable at $x$ and a vectorfield $X$ at $x$, we write $X f$ to denote $\mathrm{d} f(X)=\partial_{X} f$. Often, $X=e_{i}$, where $\left\{e_{i}\right\}$ is a basis vector field inside a local frame. The support of a function (or section) $f$ is denoted by spt $f$. Whenever we write $\mathrm{C}^{k, \alpha}$, we do not assume $\mathrm{C}^{k, \alpha}$ with global control of the norm but rather, only $\mathrm{C}^{k, \alpha}$ regularity locally. 
2.2. Manifolds and vector bundles. Let $\mathcal{M}$ be a smooth, connected manifold and $\mathrm{g}$ be a metric on $\mathcal{M}$ that is at least $\mathrm{C}^{0,1}$ (locally Lipschitz). By $\rho$ denote the distance metric induced by $g$ and by $\mu$ the induced volume measure.

Throughout this paper, we assume that $(\mathcal{M}, \mathrm{g})$ is complete, by which we mean that $(\mathcal{M}, \rho)$ is a complete metric space. $\mathrm{By} \mathrm{B}(x, r)$ or $\mathrm{B}_{r}(x)$, we denote a $\rho$-metric open ball of radius $r>0$ centred at $x \in \mathcal{M}$. For an arbitrary ball $\mathrm{B}$, we denote its radius by $\operatorname{rad}(\mathrm{B})$. We recall that by the Hopf-Rinow theorem, the condition of completeness is equivalent to the fact that $\overline{\mathrm{B}(x, r)}$ is compact for any $x \in \mathcal{M}$ and $r<\infty$.

By $\mathcal{V}$, we denote a smooth complex vector bundle of dimension $\operatorname{dim} \mathcal{V}=N$ over $\mathcal{M}$ with a metric $\mathrm{h}$ that is at least $\mathrm{C}^{0,1}$. We let $\pi_{\mathcal{V}}: \mathcal{V} \rightarrow \mathcal{M}$ be the bundle projection map. We define the space of $\mu$-measurable sections of $\mathcal{V}$ by $\Gamma(\mathcal{V})$. Using the Riemannian measure $\mu$ and the bundle metric $\mathrm{h}$, we define the standard $\mathrm{L}^{p}$ spaces which we denote by $\mathrm{L}^{p}(\mathcal{V})$.

Let us now assume that $\nabla$ is a connection on $\mathcal{V}$, compatible with $\mathrm{h}$ almost-everywhere. By $\nabla_{2}: \mathcal{D}\left(\nabla_{2}\right) \rightarrow \mathrm{L}^{2}\left(\mathrm{~T}^{*} \mathcal{M} \otimes \mathcal{V}\right)$, denote the operator $\nabla$ with domain

$$
\mathcal{D}\left(\nabla_{2}\right)=\left\{u \in \mathrm{C}^{\infty} \cap \mathrm{L}^{2}(\mathcal{V}): \nabla u \in \mathrm{L}^{2}\left(\mathrm{~T}^{*} \mathcal{M} \otimes \mathcal{V}\right)\right\}
$$

Then, $\nabla_{2}$ is densely-defined and closable, and we define the Sobolev space $\mathrm{W}^{1,2}(\mathcal{V})=$ $\mathcal{D}\left(\overline{\nabla_{2}}\right)$, with norm $\|u\|_{\mathrm{W}^{1,2}}^{2}=\left\|\overline{\nabla_{2}} u\right\|^{2}+\|u\|^{2}$. Moreover, recall that the divergence operator is then div $=-\bar{\nabla}_{2}^{*}$. It is well known that $\mathrm{C}_{\mathrm{c}}^{\infty}(\mathcal{V})$ is dense in $\mathrm{W}^{1,2}(\mathcal{V})$ and when $\mathrm{g}$ is smooth, that $\mathrm{C}_{\mathrm{c}}^{\infty}\left(\mathrm{T}^{*} \mathcal{M} \otimes \mathcal{V}\right)$ is dense in $\mathcal{D}($ div) (see [5]). In what is to follow, we will sometimes write $\nabla$ in place of $\overline{\nabla_{2}}$.

We shall require the following concept of growth of the measure $\mu$ in later analysis.

Definition 2.1 (Exponential volume growth). We say that $(\mathcal{M}, \mathrm{g}, \mu)$ has exponential volume growth if there exists $c_{E} \geq 1, \kappa, c>0$ such that

$$
0<\mu(\mathrm{B}(x, t r)) \leq c t^{\kappa} \mathrm{e}^{c_{E} t r} \mu(\mathrm{B}(x, r))<\infty,
$$

for every $t \geq 1, r>0$ and $x \in \mathcal{M}$.

We shall also require the following property.

Definition 2.2 (Local Poincaré inequality). We say that $\mathcal{M}$ satisfies a local Poincaré inequality if there exists $c_{P} \geq 1$ such that for all $f \in \mathrm{W}^{1,2}(\mathcal{M})$,

$$
\left\|f-\left(f_{B} f d \mu_{\mathrm{g}}\right)\right\|_{\mathrm{L}^{2}(B)} \leq c_{P} \operatorname{rad}(B)\|f\|_{\mathrm{W}^{1,2}(B)}
$$

for all balls $B$ in $\mathcal{M}$ such that $\operatorname{rad}(B) \leq 1$.

This growth assumption as well as the local Poincaré inequality are very natural, i.e., if the Ricci curvature Ric of a smooth g satisfies $\operatorname{Ric}_{\mathrm{g}} \geq \eta \mathrm{g}$ for some $\eta \in \mathbb{R}$, then by the Bishop-Gromov comparison theorem (c.f. Chapter 9 in [24]), ( $\left.\mathrm{E}_{\mathrm{loc}}\right)$ and $\left(\mathrm{P}_{\text {loc }}\right)$ are both satisfied. 
As for the vector bundle $\mathcal{V}$, we require the following uniformly local Euclidean structure, referred to as generalised bounded geometry or $G B G$ following terminology from [6].

Definition 2.3 (Generalised Bounded Geometry). We say that $(\mathcal{M}, \mathrm{h})$ satisfies generalised bounded geometry, or GBG for short, if there exist $\rho>0$ and $C \geq 1$ such that, for each $x \in \mathcal{M}$, there exists a continuous local trivialisation $\psi_{x}: \mathrm{B}(x, \rho) \times$ $\mathbb{C}^{N} \rightarrow \pi_{\mathcal{V}}^{-1}(\mathrm{~B}(x, \rho))$ satisfying

$$
C^{-1}\left|\psi_{x}^{-1}(y) u\right|_{\delta} \leq|u|_{\mathrm{h}(y)} \leq C\left|\psi_{x}^{-1}(y) u\right|_{\delta},
$$

for all $y \in \mathrm{B}(x, \rho)$, where $\delta$ denotes the usual inner product in $\mathbb{C}^{N}$ and $\psi_{x}^{-1}(y) u=$ $\psi_{x}^{-1}(y, u)$ is the pullback of the vector $u \in \mathcal{V}_{y}$ to $\mathbb{C}^{N}$ via the local trivialisation $\psi_{x}$ at $y \in \mathrm{B}(x, \rho)$. We call $\rho$ the $\mathrm{GBG}$ radius.

We remark that, unlike in [6], we do not ask for the trivialisations to be smooth. A trivialisation satisfying the above condition is said to be a $G B G$ chart and a set of trivialisations $\left\{\psi_{x}: x \in \mathcal{M}\right\}$ a $G B G$ atlas. For each GBG chart $\psi_{x}$, the associated $G B G$ frame is then

$$
\left\{e^{i}(y)=\psi_{x}\left(y, \hat{e}^{i}\right):\left\{\hat{e}^{i}\right\} \text { standard basis for } \mathbb{C}^{N}\right\} .
$$

If these trivialisations have higher regularity, i.e. the trivialisations are $\mathrm{C}^{k, \alpha}$ for some $k \geq 0$ and $\alpha \in(0,1)$, then we refer to this aforementioned terminology as a $\mathrm{C}^{k, \alpha}$ GBG chart/atlas/frame respectively.

Like exponential growth, generalised bounded geometry is a geometrically natural condition. In the case that the metric $\mathrm{g}$ is smooth and complete, under the assumption $\operatorname{inj}(\mathcal{M}, \mathrm{g}) \geq \kappa>0$ and $\operatorname{Ric}_{\mathrm{g}} \geq \eta \mathrm{g}$ for some $\kappa>0$ and $\eta \in \mathbb{R}$, the bundle of $(p, q)$-tensors satisfies GBG. See Theorem 1.2 in [17] and Corollary 6.5 in [6].

2.3. Functional calculus. In this section, we introduce some notions from operator theory and functional calculi that will be of relevance in subsequent sections.

Let $\mathscr{H}$ be a Hilbert space, and $T: \mathcal{D}(T) \subset \mathscr{H} \rightarrow \mathscr{H}$ a self-adjoint operator. Indeed, by the spectral theorem (see [18], Chapter 6, §5), for every Borel function $b: \mathbb{R} \rightarrow \mathbb{R}$, we can define and estimate the operator $b(T)$. However, we shall only consider symbols $b$ which are holomorphic on a neighbourhood of $\mathbb{R}$, in which case $b(T)$ is obtained by the Riesz-Dunford functional calculus as we now explain.

For $\omega \in(0, \pi / 2)$ and $\sigma \in(0, \infty)$, define

$$
\mathrm{S}_{\omega, \sigma}^{\mathrm{o}}:=\left\{x+i y: y^{2}<\tan ^{2} \omega x^{2}+\sigma^{2}\right\},
$$

We say that a function $\psi \in \Psi\left(\mathrm{S}_{\omega, \sigma}^{o}\right)$ if it is holomorphic on $\mathrm{S}_{\omega, \sigma}^{o}$ and there exists an $\alpha>0, C>0$ such that

$$
|\psi(\zeta)| \leq \frac{C}{|\zeta|^{\alpha}}
$$


Letting the curve $\gamma$ denote $\left\{y^{2}=\tan ^{2}(\omega / 2) x^{2} / 2+\sigma^{2} / 2\right\}$, oriented counter-clockwise inside $\mathrm{S}_{\omega, \sigma}^{\circ}$, the Riesz-Dunford functional calculus is

$$
\psi(T) u=\frac{1}{2 \pi \mathrm{i}} \oint_{\gamma} \psi(\zeta) \mathrm{R}_{T}(\zeta) u d \zeta,
$$

for each $u \in \mathscr{H}$, with $\mathrm{R}_{T}(\zeta)=(\zeta \mathrm{I}-T)^{-1}$ and where the integral converges absolutely as Riemann-sums.

We say that a holomorphic function $f \in \operatorname{Hol}^{\infty}\left(\mathrm{S}_{\omega, \sigma}^{\circ}\right)$ if there exists $C>0$ such that $\|f(\zeta)\|_{\infty} \leq C$. For such a function, there exists a uniformly bounded $\psi_{n} \in \Psi\left(\mathrm{S}_{\omega, \sigma}^{\circ}\right)$ such that $\psi_{n} \rightarrow f$ pointwise, and the functional calculus is defined as

$$
f(T) u=\lim _{n \rightarrow \infty} \psi_{n}(T) u
$$

for $u \in \mathscr{H}$, which converges due to the fact that $T$ is self-adjoint, and is independent of the sequence $\psi_{n}$.

These details are obtained as a special case of the functional calculus for the socalled $\omega$-bisectorial operators. A detailed exposition can be found in [22] by Morris and for $\omega$-sectorial operators in [1] by Albrecht, Duong, and McIntosh and [16] by Haase.

2.4. The main theorem. We assume that the manifold $(\mathcal{M}, \mathrm{g})$ is complete and that both $\mathrm{g}$ and $\mathrm{h}$ are at least $\mathrm{C}^{0,1}$.

Let $\mathrm{D}$ be a first-order differential operator on $\mathrm{C}^{\infty}(\mathcal{V})$. By this, we mean that, inside each frame $\left\{e^{i}\right\}$ for $\mathcal{V}$ and $\left\{v_{j}\right\}$ for TM near $x$, there exist coefficients $\alpha_{l}^{j k}$ and terms $\omega_{q}^{p}$ (not necessarily smooth) such that

$$
\mathrm{D} u=\left(\alpha_{l}^{j k} \nabla_{v_{j}} u_{k}+u_{i} \omega_{l}^{i}\right) e^{l},
$$

where $u=u_{i} e^{i} \in \mathrm{C}^{\infty}(\mathcal{V})$.

We consider two essentially self-adjoint first-order differential operators D and D , and with slight abuse of notation we use this notation for their self-adjoint extensions.

In establishing our main perturbation estimate from $\mathrm{D}$ to $\tilde{\mathrm{D}}$ on $\mathcal{V} \rightarrow \mathcal{M}$, we will make the following hypotheses:

(A1) $\mathcal{M}$ and $\mathcal{V}$ are finite dimensional, quantified by $\operatorname{dim} \mathcal{M}<\infty$ and $\operatorname{dim} \mathcal{V}<\infty$,

(A2) $(\mathcal{M}, g)$ has exponential volume growth as defined in Definition 2.1, quantified by $c<\infty, c_{E}<\infty$ and $\kappa<\infty$ in $\left(\mathrm{E}_{\mathrm{loc}}\right)$,

(A3) A local Poincaré inequality $\left(\mathrm{P}_{\text {loc }}\right)$ holds on $\mathcal{M}$ as in Definition 2.2 quantified by $c_{P}<\infty$

(A4) $\mathrm{T}^{*} \mathcal{M}$ has $\mathrm{C}^{0,1}$ GBG frames $\nu_{j}$ quantified by $\rho_{\mathrm{T}^{*} \mathcal{M}}>0$ and $C_{\mathrm{T}^{*} \mathcal{M}}<\infty$ in Definition 2.3, with regularity $\left|\nabla \nu_{j}\right|<C_{G, \mathrm{~T}^{*} \mathcal{M}}$ with $C_{G, \mathrm{~T}^{*} \mathcal{M}}<\infty$ almosteverywhere,

(A5) $\mathcal{V}$ has $\mathrm{C}^{0,1}$ GBG frames $e_{j}$ quantified by $\rho_{\mathcal{V}}>0$ and $C_{\mathcal{V}}<\infty$ in Definition 2.3 , with regularity $\left|\nabla e_{j}\right|<C_{G, \mathcal{V}}$ with $C_{G, \mathcal{V}}<\infty$ almost-everywhere, 
(A6) $\mathrm{D}$ is a first-order $\mathrm{PDO}$ with $\mathrm{L}^{\infty}$ coefficients. In particular, $[\mathrm{D}, \eta]$ is a pointwise multiplication operator on almost-every fibre $\mathcal{V}_{x}$, and there exists $c_{\mathrm{D}}>0 \mathrm{such}$ that

$$
|[\mathrm{D}, \eta] u(x)| \leq c_{\mathrm{D}} \operatorname{Lip} \eta(x)|u(x)|
$$

for almost-every $x \in \mathcal{M}$, every bounded Lipschitz function $\eta$, and where $\operatorname{Lip} \eta(x)$ is the pointwise Lipschitz constant.

(A7) D satisfies $\left|\mathrm{D} e_{j}\right| \leq C_{D, \mathcal{V}}$ with $C_{D, \mathcal{V}}<\infty$ almost-everywhere inside each GBG frame $\left\{e_{j}\right\}$,

(A8) $\mathrm{D}$ and $\tilde{\mathrm{D}}$ both have domains $\mathrm{W}^{1,2}(\mathcal{V})$ with $\mathrm{C} \geq 1$ the smallest constants satisfying

$\mathrm{C}^{-1}\|u\|_{\mathrm{D}} \leq\|u\|_{\mathrm{W}^{1,2}} \leq \mathrm{C}\|u\|_{\mathrm{D}} \quad$ and $\quad \mathrm{C}^{-1}\|u\|_{\tilde{\mathrm{D}}} \leq\|u\|_{\mathrm{W}^{1,2}} \leq \mathrm{C}\|u\|_{\tilde{\mathrm{D}}}$

(A9) D satisfies the Riesz-Weitzenböck condition

$$
\left\|\nabla^{2} u\right\| \leq c_{W}\left(\left\|\mathrm{D}^{2} u\right\|+\|u\|\right)
$$

with $c_{W}<\infty$.

The implicit constants in our perturbation estimates will be allowed to depend on

$$
\begin{aligned}
\mathrm{C}(\mathcal{M}, \mathcal{V}, \mathrm{D}, \tilde{\mathrm{D}})=\max \left\{\operatorname{dim} \mathcal{M}, \operatorname{dim} \mathcal{V}, c, c_{E}, \kappa, c_{P}, \rho_{\mathrm{T}^{*} \mathcal{M}}, C_{\mathrm{T}^{*} \mathcal{M}}, C_{G, \mathrm{~T}^{*} \mathcal{M}},\right. \\
\left.\rho_{\mathcal{V}}, C_{\mathcal{V}}, C_{G, \mathcal{V}}, c_{\mathrm{D}}, C_{\mathrm{D}, \mathcal{V}}, \mathrm{C}, c_{W}\right\}<\infty
\end{aligned}
$$

In section $\S 4$ and $\S 5$, we prove the following theorem.

Theorem 2.4. Let $(\mathcal{M}, \mathrm{g})$ be a smooth Riemannian manifold with $\mathrm{g}$ that is $\mathrm{C}^{0,1}$, complete, and satisfying $\left(\mathrm{E}_{\mathrm{loc}}\right)$ and $\left(\mathrm{P}_{\mathrm{loc}}\right)$. Let $(\mathcal{V}, \mathrm{h}, \nabla)$ be a smooth vector bundle with $\mathrm{C}^{0,1}$ metric $\mathrm{h}$ and connection $\nabla$ that are compatible almost-everywhere.

Let $\mathrm{D}, \tilde{\mathrm{D}}$ be self-adjoint operators on $\mathrm{L}^{2}(\mathcal{V})$ and assume the hypotheses (A1)-(A9) on $\mathcal{M}, \mathcal{V}, \mathrm{D}$ and $\tilde{\mathrm{D}}$. Moreover, assume that

$$
\tilde{\mathrm{D}} \psi=\mathrm{D} \psi+A_{1} \nabla \psi+\operatorname{div} A_{2} \psi+A_{3} \psi,
$$

holds in a distributional sense for $\psi \in \mathrm{W}^{1,2}(\mathcal{V})$, where

$$
\begin{aligned}
& A_{1} \in \mathrm{L}^{\infty}\left(\mathcal{L}\left(\mathrm{T}^{*} \mathcal{M} \otimes \mathcal{V}, \mathcal{V}\right)\right), \\
& A_{2} \in \mathrm{L}^{\infty}\left(\mathrm{W}^{1,2}(\mathcal{V}), \mathcal{D}(\operatorname{div})\right), \text { and } \\
& A_{3} \in \mathrm{L}^{\infty}(\mathcal{L}(\mathcal{V})),
\end{aligned}
$$

and let $\|A\|_{\infty}=\left\|A_{1}\right\|_{\infty}+\left\|A_{2}\right\|_{\infty}+\left\|A_{3}\right\|_{\infty}$.

Then, for each $\omega \in(0, \pi / 2)$ and $\sigma \in(0, \infty]$, whenever $f \in \operatorname{Hol}^{\infty}\left(\mathrm{S}_{\omega, \sigma}^{\circ}\right)$, we have the perturbation estimate

$$
\|f(\tilde{\mathrm{D}})-f(\mathrm{D})\|_{\mathrm{L}^{2}(\mathcal{V}) \rightarrow \mathrm{L}^{2}(\mathcal{V})} \lesssim\|f\|_{\mathrm{L}^{\infty}\left(\mathrm{S}_{\omega, \sigma}\right)}\|A\|_{\infty},
$$

where the implicit constant depends on $\mathrm{C}(\mathcal{M}, \mathcal{V}, \mathrm{D}, \tilde{\mathrm{D}})$.

Remark 2.5. The assumption of self-adjointness of the operators $\mathrm{D}$ and $\tilde{\mathrm{D}}$ in Theorem 2.4 can be relaxed, as we only use this to deduce quadratic estimates for $\mathrm{D}$ and $\tilde{\mathrm{D}}$. For example, it suffices to assume that $\mathrm{D}$ and $\tilde{\mathrm{D}}$ are similar in $\mathrm{L}^{2}$ to self-adjoint operators. 
Remark 2.6. Although our motivation and key application in is in the case that $\mathrm{D}$ and $\tilde{\mathrm{D}}$ correspond to the Atiyah-Singer Dirac operators on a Spin manifold corresponding to two different metrics, we allow for greater generality in our main theorem since we anticipate it to have a much broader set of applications. For instance, in the study of particle physics, twisted bundles and their associated twisted Dirac operators are of significance and we expect that such situations might also be analysed by our main theorem. For readers interested in such operators, we hope that $\S 3$ will serve as a guideline to how hypotheses (A1)-(A9) can be shown to be satisfied.

\section{Applichtions to the Atiyah-Singer Dirac operator}

Let $\mathcal{M}$ be a smooth manifold with a $\mathrm{C}^{0,1}$ (locally Lipschitz) metric g. We let $\Omega \mathcal{M}$ denote the bundle of differential forms and on fixing a Clifford product $\triangle$, we let $\Delta \mathcal{M}=\Delta \mathrm{T} \mathcal{M}$ denote the Clifford bundle. Recall that $\Delta \mathcal{M} \cong \Omega \mathcal{M}$ as a vector space. Moreover, we remind the reader that we identify vectorfields and derivations throughout, so $X f$ means the directional derivative $\partial_{X} f$ where $X$ is a vectorfield and $f$ is a scalar function.

Fix a frame $\left\{v_{j}\right\}$ near $x$, let $\mathrm{g}_{i j}=\mathrm{g}\left(v_{i}, v_{j}\right)$ and define $w_{k l}^{i}$ at points where $\mathrm{g}$ is differentiable inside the frame by

$$
w_{k l}^{i}=\frac{1}{2} \mathrm{~g}^{i m}\left(\partial_{v_{l}} \mathrm{~g}_{m k}+\partial_{v_{k}} \mathrm{~g}_{m l}-\partial_{v_{m}} \mathrm{~g}_{k l}+c_{m k l}+c_{m l k}-c_{k l m}\right)
$$

where $c_{k l m}=\mathrm{g}\left(\left[v_{k}, v_{l}\right], v_{m}\right)$ are the commutation coefficients and $[\cdot, \cdot]$ is the Lie derivative. Let $\omega_{i}^{a}=w_{j i}^{a} e^{j}$ be the connection 1-form, and define $\nabla^{\mathrm{g}} v_{j}=\omega_{j}^{a} \otimes v_{a}$. Thus, we obtain the Levi-Civita connection almost-everywhere in $\mathcal{M}$ as a map $\nabla^{\mathrm{g}}$ : $\mathrm{C}^{\infty}(\mathrm{T} \mathcal{M}) \rightarrow \Gamma\left(\mathrm{T}^{*} \mathcal{M} \otimes \mathrm{T} \mathcal{M}\right)$. Note that since $\mathrm{g}$ is only locally Lipschitz, we have that smooth sections are mapped to locally bounded (1,1)-tensors. When the context is clear, we often simply denote $\nabla^{\mathrm{g}}$ by $\nabla$.

A manifold $(\mathcal{M}, \mathrm{g})$ is said to be $\operatorname{Spin}$ if it admits a spin structure $\xi: \mathrm{P}_{\text {Spin }}(\mathrm{T} \mathcal{M}) \rightarrow$ $\mathrm{P}_{\mathrm{SO}}(\mathrm{T} \mathcal{M})$, i.e., a $2-1$ covering of the frame bundle. It is well known that this occurs if and only if the first and second Stiefel-Whitney classes of the tangent bundle vanish. The triviality of the first Stiefel-Whitney class is equivalent to the orientability of $\mathcal{M}$.

For the case of $\mathcal{M}=\mathbb{R}^{n}$ with $\mathrm{g}=\delta$, the usual Euclidean inner product, we let $\Delta \mathbb{R}^{n}$ denote linear space of standard complex spinors of dimension $2^{\left\lfloor\frac{n}{2}\right\rfloor}$. In odd dimensions, this space corresponds to the non-trivial minimal complex irreducible representation $\eta: \operatorname{Spin}_{n} \rightarrow \mathcal{L}\left(\Delta \mathbb{R}^{n}\right)$, where $\operatorname{Spin}_{n}$ is the spin group, the double cover of $\mathrm{SO}_{n}$, and in even dimension to $\eta=\eta_{+} \oplus \eta_{-}$where $\eta_{ \pm}: \operatorname{Spin}_{n} \rightarrow \mathcal{L}\left(\boldsymbol{\Delta}_{ \pm} \mathbb{R}^{n}\right)$ are the representations of the positive/negative half spinors. For example, see [19]. We define the standard (complex) Spin bundle to be

$$
\not \Delta \mathcal{M}=\mathrm{P}_{\mathrm{Spin}}(\mathrm{T} \mathcal{M}) \times_{\eta} \not \Delta \mathbb{R}^{n}
$$


as the bundle with fibre $\not \Delta \mathbb{R}^{n}$ associated to $\mathrm{P}_{\text {Spin }}(\mathrm{T} \mathcal{M})$ via $\eta$. We note that this is the bundle with transition functions $\left(\eta \circ T_{\alpha \beta}\right)$ on $\Omega_{\alpha} \cap \Omega_{\beta} \neq \varnothing$ for $\Omega_{\alpha}$ and $\Omega_{\beta}$ open sets, where $T_{\alpha \beta}: \Omega_{\alpha} \cap \Omega_{\beta} \rightarrow \operatorname{Spin}_{n}$ are transition functions for $\mathrm{P}_{\mathrm{Spin}}(\mathrm{T} \mathcal{M})$.

The representation $\eta$ induces an action $\cdot: \Delta \mathcal{M} \rightarrow \Delta \mathcal{M}$. When $n$ is odd, there are two such multiplications up to equivalence opposite from each other, and for $n$ even, there is exactly one up to equivalence. Fixing such a Clifford action, $\Delta \mathcal{M}$ has an induced hermitian metric $\langle\cdot, \cdot\rangle_{*}$, pointwise unique up to scale satisfying $\langle X \cdot \varphi, \psi\rangle_{*}=$ $-\langle\varphi, X \cdot \psi\rangle_{*}$ for all $X \in \mathrm{T}_{x} \mathcal{M}$ and $\varphi, \psi \in \Delta_{x} \mathcal{M}$ for every $x \in \mathcal{M}$. See Proposition 1.2 .1 and 1.2 .3 in [15].

Let $E\left(e_{1}, \ldots, e_{n}\right)$ be an orthonormal frame for TM and $\left\{\phi_{\alpha}\right\}$ be the induced orthonormal spin frame on $\Delta \Delta \mathcal{M}$. Let $\omega_{i}^{a}$ be the connection 1-form in $E$ and define the connection $\nabla: \mathrm{C}^{\infty}(\Delta \mathcal{M}) \rightarrow \mathrm{L}_{\mathrm{loc}}^{\infty}\left(\mathrm{T}^{*} \mathcal{M} \otimes \Delta \mathcal{M}\right)$ by writing

$$
\nabla \phi_{\alpha}=\frac{1}{2} \sum_{b<a} \omega_{b}^{a} \otimes\left(e_{b} \cdot \mathrm{e}_{a} \cdot \phi_{\alpha}\right) .
$$

This connection satisfies the two following properties:

(i) it is almost-everywhere compatible with the induced spinor metric $\langle\cdot, \cdot\rangle_{*}$, and

(ii) it is a module derivation: whenever $X \in \mathrm{C}^{\infty}(\mathrm{T} \mathcal{M})$,

$$
\nabla_{X}(\omega \cdot \psi)=\left(\nabla_{X} \omega\right) \cdot \psi+\omega \cdot\left(\nabla_{X} \psi\right)
$$

holds almost-everywhere for every $\omega \in \mathrm{C}^{\infty}(\Delta \mathcal{M})$ and $\psi \in \mathrm{C}^{\infty}(\Delta \mathcal{M})$.

We refer the reader to $§ 1.2$ in [15] for a exposition of these ideas, as well as Chapter $2, \S 3$ to $\S 5$ in [19] for a detailed overview, noting that their proofs in the smooth setting hold in our setting almost-everywhere.

Write

$$
\omega_{E}^{2}=\frac{1}{2} \sum_{b<a} \omega_{b}^{a} \otimes e_{b} \cdot e_{a}
$$

to denote the lifting of the connection 2-form $\frac{1}{2} \sum_{b<a} \omega_{b}^{a} \otimes e_{b} \wedge e_{a}$ to $\Delta \mathcal{M}$, and where $E$ is used to denote the dependence on the frame $E\left(e_{1}, \ldots, e_{n}\right)$. By $\not_{\mathrm{g}}$ denote the associated Atiyah-Singer Dirac operator given by the expression

$$
\not D_{\mathrm{g}} \phi_{\alpha}=e^{j} \cdot \nabla_{e_{j}} \phi_{\alpha}=e^{j} \cdot \omega_{E}^{2}\left(e_{j}\right) \cdot \phi_{\alpha},
$$

so that $\not_{\mathrm{g}}\left(\psi^{\alpha} \phi_{\alpha}\right)=\left(\nabla_{e_{j}} \psi^{\alpha}\right) e^{j} \cdot \phi_{\alpha}+\psi^{\alpha} e^{j} \cdot \nabla_{e_{j}} \phi_{\alpha}$. Note that,

$$
\not_{\mathrm{g}}(\eta \psi)=(\mathrm{d} \eta) \cdot \psi+\eta \not_{\mathrm{g}}(\psi)
$$

for every $\eta \in \mathrm{C}^{\infty}(\mathcal{M})$ and $\psi \in \mathrm{C}^{\infty}(\Delta \mathcal{M})$ and, as a consequence of the aforementioned module-derivation property of the connection $\nabla$ on $\Delta \mathcal{M}$,

$$
\not D_{\mathrm{g}}(\omega \cdot \psi)=\left(\mathrm{D}_{H} \omega\right) \cdot \psi-\omega \cdot \not_{\mathrm{g}} \psi-2 \nabla_{\omega^{\sharp}} \psi
$$

for all $\omega \in \mathrm{C}^{\infty}(\Delta \mathcal{M})$ and $\psi \in \mathrm{C}^{\infty}(\Delta \mathcal{M})$, where $\mathrm{D}_{H}=\mathrm{d}+\mathrm{d}^{*}$ is the Hodge-Dirac operator, and $\sharp: \mathrm{T}^{*} \mathcal{M} \rightarrow \mathrm{T} \mathcal{M}$ given by $\omega^{\sharp}=\mathrm{g}(\omega, \cdot)$. 
Next, let $(\mathcal{N}, \mathrm{h})$ be another Spin manifold with a smooth differentiable structure and $\mathrm{h}$ at least $\mathrm{C}^{0,1}$. Suppose that $\zeta: \mathcal{M} \rightarrow \mathcal{N}$ is a $\mathrm{C}^{1,1}$-diffeomorphism and let $\Delta \mathcal{N}$ denote the complex standard spin bundle of $\mathcal{N}$ obtained via $\eta$. Following [8], we define an induced unitary map of spinors $\psi: \Delta \mathcal{M} \rightarrow \Delta \mathcal{N}$. Let $P=\zeta_{*}$ : $\mathrm{T} \mathcal{M} \rightarrow \mathrm{TN}$. Then, the pullback metric is $\tilde{\mathrm{g}}(u, v)=\mathrm{h}(P u, P v)$ and we have that $\tilde{\mathrm{g}}(u, v)=\mathrm{g}\left(\left(P_{\mathrm{g}}^{*} P\right) u, v\right)$, where $P_{\mathrm{g}}^{*}$ is the adjoint of $P$, and this expression is readily checked to be a metric of class $\mathrm{C}^{0,1}$. On letting $\mathrm{U}=P\left(P_{\mathrm{g}}^{*} P\right)^{-\frac{1}{2}}$, we have that $\mathrm{h}(\mathrm{U} u, \mathrm{U} v)=\mathrm{g}(u, v)$. So, $\mathrm{U}:(\mathrm{T} \mathcal{M}, \mathrm{g}) \rightarrow(\mathrm{T} \mathcal{N}, \mathrm{h})$ is an isometry of class $\mathrm{C}^{0,1}$. By $\mathrm{U}(x)$, we denote the induced linear isometry $\mathrm{U}(x):\left(\mathrm{T}_{x} \mathcal{M}, \mathrm{g}\right) \rightarrow\left(\mathrm{T}_{\zeta(x)} \mathcal{N}, \mathrm{h}\right)$.

Since $\zeta$ is a homeomorphism, an open set $\Omega \subset \mathcal{M}$ is contractible if and only if $\zeta(\Omega) \subset \mathcal{N}$ is contractible. For an orthonormal frame $E\left(e_{1}, \ldots, e_{n}\right) \in \mathrm{P}_{\mathrm{SO}}(\mathrm{T} \mathcal{M})$ in $\Omega$, we obtain $\mathrm{U} E\left(e_{1}, \ldots, e_{n}\right) \in \mathrm{P}_{\mathrm{SO}}(\mathrm{T} \mathcal{N})$. Lifting $E$ and $\mathrm{U} E$ through the spin structures locally, we obtain two possible maps $\chi_{\text {Spin }, \Omega}: \mathrm{P}_{\text {Spin }}(\mathcal{M}) \rightarrow \mathrm{P}_{\text {Spin }}(\mathcal{N})$ differing by a sign. We say that the bundles $\Delta \mathcal{M}$ and $\Delta \mathcal{N}$ are compatible if $\psi_{\text {Spin, } \Omega}$ induces a well-defined global unitary map $\forall: \Delta \mathcal{M} \rightarrow \Delta \mathcal{N}$. By examining the local expression, we see that $\forall: \not \Delta \mathcal{M} \rightarrow \not \Delta \mathcal{N}$ and $\emptyset^{-1}: \Delta \mathcal{N} \rightarrow \not \Delta \mathcal{M}$ are $\mathrm{C}^{0,1}$ maps.

Finally, we say that $\mathrm{g}$ and $\mathrm{h}$ are $C$-close for some $C \geq 1$, if for all $x \in \mathcal{M}$,

$$
C^{-1}|u|_{\mathrm{g}(x)} \leq\left|\zeta_{*} u\right|_{\mathrm{h}(\zeta(x))} \leq C|u|_{\mathrm{g}(x)} .
$$

Define

$$
C_{L}=\inf \{C \geq 1: \mathrm{g} \text { and } \mathrm{h} \text { are } C \text {-close }\} \quad \text { and } \quad \rho_{M}\left(\mathrm{~g}, \zeta^{*} \mathrm{~h}\right)=\log \left(C_{L}\right) .
$$

The map $\rho_{M}$ is readily verified to be a distance-metric on the space of metrics.

What follows is the main the result of this section. In fact, this theorem was the original motivation of this paper, whereas Theorem 2.4 is a natural generalisation. As aforementioned, we anticipate the more general result to have wider implications, particularly to Dirac operators that arise through twisting the spin bundle by other natural vector bundles. The analysis of such objects is beyond the scope of this paper and hence, we focus on the particular case of the Atiyah-Singer Dirac operator.

Theorem 3.1. Let $\mathcal{M}$ be a smooth Spin manifold with smooth, complete metric $\mathrm{g}$ with Levi-Civita connection $\nabla^{\mathrm{g}}$, let $\mathcal{N}$ be a smooth Spin manifold with a $\mathrm{C}^{0,1}$ metric $\mathrm{h}$, and $\zeta: \mathcal{M} \rightarrow \mathcal{N}$ a $\mathrm{C}^{1,1}$-diffeomorphism with $\rho_{M}\left(\mathrm{~g}, \zeta^{*} \mathrm{~h}\right) \leq 1$. We assume that the spin bundles $\triangle \mathcal{M}$ and $\Delta \mathcal{N}$ are compatible. Moreover, suppose that the following hold:

(i) there exists $\kappa>0$ such that $\operatorname{inj}(\mathcal{M}, \mathrm{g}) \geq \kappa$,

(ii) there exists $C_{R}>0$ such that $\left|\mathrm{Ric}_{\mathrm{g}}\right| \leq C_{R}$ and $\left|\nabla^{\mathrm{g}} \mathrm{Ric}_{\mathrm{g}}\right| \leq C_{R}$,

(iii) there exists $C_{\mathrm{h}}>0$ such that $\left|\nabla^{\mathrm{g}}\left(\zeta^{*} \mathrm{~h}\right)\right| \leq C_{\mathrm{h}}$ almost-everywhere.

Then, for $\omega \in(0, \pi / 2), \sigma>0$, whenever $f \in \mathrm{Hol}^{\infty}\left(\mathrm{S}_{\omega, \sigma}^{o}\right)$, we have the perturbation estimate

$$
\left\|f\left(\not D_{\mathrm{g}}\right)-f\left(\mathrm{U}^{-1} \not D_{\mathrm{h}} \mathrm{W}\right)\right\|_{\mathrm{L}^{2} \rightarrow \mathrm{L}^{2}} \lesssim\|f\|_{\infty} \rho_{M}\left(\mathrm{~g}, \zeta^{*} \mathrm{~h}\right)
$$

where the implicit constant depends on $\operatorname{dim} \mathcal{M}$ and the constants appearing in (i)(iii). 
Remark 3.2. The map $\forall$ is the fibrewise unitary map $\Delta_{p} \mathcal{M} \rightarrow \Delta_{\zeta(p)} \mathcal{N}$. For the $\mathrm{L}^{2}(\Delta \mathcal{M}) \rightarrow \mathrm{L}^{2}(\Delta \mathcal{N})$ unitary operator $\mathrm{H}_{2}=\sqrt{\operatorname{det} \mathrm{B}} \boldsymbol{\phi}$, we also have an estimate of $\left\|f\left(\not_{\mathrm{g}}\right)-f\left(\mathrm{U}_{2}^{-1} \not_{\mathrm{h}} \mathrm{U}_{2}\right)\right\|_{\mathrm{L}^{2} \rightarrow \mathrm{L}^{2}}$ as in Theorem 3.1. Either this can be seen by inspection of the proof, noting Remark 2.5, or by using the functional calculus to write

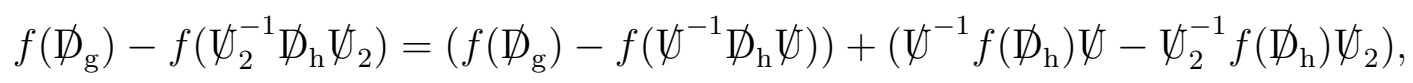

noting that the second term is straightforward to bound.

Remark 3.3. On fixing a Spin structure $\xi: \mathrm{P}_{\mathrm{Spin}}(\mathrm{T} \mathcal{M}) \rightarrow \mathrm{P}_{\mathrm{SO}}(\mathrm{T} \mathcal{M})$, we obtain an induced $\xi^{\prime}=\mathrm{U} \xi:\left(\xi^{-1} \mathrm{U}^{-1} \mathrm{P}_{\mathrm{SO}}(\mathrm{T} \mathcal{N})\right) \rightarrow \mathrm{P}_{\mathrm{SO}}(\mathrm{T} \mathcal{N})$ which is a Spin structure for $\mathcal{N}$. Since $\mathrm{U}: \mathrm{P}_{\mathrm{SO}}(\mathrm{T} \mathcal{M}) \rightarrow \mathrm{P}_{\mathrm{SO}}(\mathrm{T} \mathcal{N})$ is a homeomorphism, it is an easy matter to verify that the bundles $\Delta \mathcal{N}=\left(\xi^{-1} \mathrm{U}^{-1} \mathrm{P}_{\mathrm{SO}}(\mathrm{T} \mathcal{N})\right) \times_{\eta} \Delta \mathbb{R}^{n}$ and $\Delta \mathcal{M}$ are compatible.

For the case of $\mathcal{M}=\mathcal{N}$, where $\Delta \mathcal{M}$ and $\Delta \mathcal{N}$ denote the respective bundles constructed via $\mathrm{g}$ and $\mathrm{h}$, we obtain this theorem for $\zeta=\mathrm{id}$. If further $\mathcal{M}=\mathcal{N}$ is compact, then (i)-(iii) in the hypothesis of the theorem are automatically satisfied, and thus we obtain the result under the sole geometric assumption that $\rho_{M}(\mathrm{~g}, \mathrm{~h}) \leq 1$.

Proof of Theorem 3.1. We apply Theorem 2.4, to the operators $\mathrm{D}=\not D_{\mathrm{g}}$ and $\tilde{\mathrm{D}}=$ $\bigcup^{-1} \not D_{\mathrm{h}} \boldsymbol{V}$, setting $\mathcal{V}=\not \mathcal{M}$.

The assumptions of completeness of g along with (i) and (ii) imply $\left(\mathrm{E}_{\text {loc }}\right)$ and $\left(\mathrm{P}_{\text {loc }}\right)$ immediately (see Theorem 1.1 in [23]). Moreover, there exists $r_{H}, C_{H}>0$, such that for all $x \in \mathcal{M}$ such that $\psi_{x}: B\left(x, r_{H}\right) \rightarrow \mathbb{R}^{n}$ are coordinate charts such that inside each chart, $\left\|g_{i j}\right\|_{\mathrm{C}^{2}\left(B\left(x, r_{H}\right)\right)} \leq C_{H}$ and $\mathrm{g} \simeq \psi_{x}^{*} \delta_{\mathbb{R}^{n}}$ with constant $C_{H}$. See Theorem 1.2 in [17].

This $\mathrm{C}^{2}$-control of the metric inside each $B\left(x, r_{H}\right)$ means that coordinate frames $\left\{\partial_{x_{i}}\right\}$ satisfy $\left|\nabla \partial_{x_{i}}\right| \lesssim 1$ and $\left|\nabla^{2} \partial_{x_{i}}\right| \lesssim 1$. On orthonormalisation of these frames in each $B\left(x, r_{H}\right)$ via the Gram-Schmidt algorithm yields frames $\left\{e_{i}\right\}$ for $\mathrm{T} \mathcal{M},\left\{e^{i}\right\}$ for $\mathrm{T}^{*} \mathcal{M}$ (the dual frame), and $\left\{\phi_{\alpha}\right\}$ for $\Delta \mathcal{M}$. These are smooth GBG frames with constant $C_{\mathrm{T}^{*} \mathcal{M}}=C_{\triangle \mathcal{M}}=1$, and with $\left|\nabla e_{j}\right|,\left|\nabla^{2} e_{j}\right| \lesssim 1$ and $\left|\nabla \phi_{\alpha}\right|,\left|\nabla^{2} \phi_{\alpha}\right| \lesssim 1$. The constants only depend on (i) and (ii). Thus, we have verified the hypotheses (A1)-(A5).

The hypothesis (A6) follows with $\mathrm{C}^{\infty}$ coefficients due to the derivation property (3.5) of $\not D_{\mathrm{g}}$ with constant $C_{D}=1$, and $(A 7)$ follows from the fact that $\left|\not \phi_{\mathrm{g}} \phi_{\alpha}\right| \lesssim\left|\nabla \phi_{\alpha}\right| \lesssim 1$.

The hypothesis (A8) is proved in $\S 3.1$ as Proposition 3.6, which makes use of the completeness of g, $C$-closeness of h to g and the geometric assumptions (i) and (ii) The hypothesis (A9) is proved in $\S 3.4$ as Proposition 3.18. It depends on the crucial covering Lemma 3.5 which is a consequence of completeness of $g$ coupled with (i) and (ii).

The remaining hypothesis to verify in Theorem 2.4 is the perturbation structure 1.2 , which is done in $\S 3.3$. 
Through the remaining sections, we assume the hypothesis of Theorem 3.1 to hold.

3.1. The domain of the Dirac operator as the Spinor Sobolev space. In this section, we establish the essential-self adjointness of $\not_{\mathrm{g}}$ and $\not_{\mathrm{h}}$. By the smoothness of g, it is well known that this operator, and all of its positive powers, are essentiallyself adjoint. For instance, see [11]. Thus, we focus only on $\nsupseteq_{\mathrm{h}}$ which arises from the lower regularity metric.

First, we assert $\not_{\mathrm{h}}$ is a symmetric operator on $\mathrm{C}_{\mathrm{c}}^{\infty}(\Delta \mathcal{N})$. This is immediate since we assume that $\mathrm{h}$ is at least $\mathrm{C}^{0,1}$, and therefore, the remaining divergence term in when computing the symmetry pointwise almost-everywhere is the divergence of a compactly supported Lipschitz vectorfield. A particular consequence of symmetry is that $\not_{\mathrm{h}}$ is a closable operator by the density of $\mathrm{C}_{\mathrm{c}}^{\infty}(\boldsymbol{\Delta} \mathcal{N})$ in $\mathrm{L}^{2}(\not \mathcal{N})$. Operator theory yields that $\overline{D_{\mathrm{h}}}=\not_{\mathrm{h}}^{* *}$. With these observations in mind, we prove the following.

Proposition 3.4. The operator $\not_{\mathrm{h}}$ on $\mathrm{C}_{\mathrm{c}}^{\infty}(\Delta \mathcal{N})$ is essentially self-adjoint.

Proof. The conclusion is established if we prove that $\mathrm{C}_{\mathrm{c}}^{\infty}(\not \boldsymbol{\mathcal { N }})$ is dense in

$$
\mathcal{D}\left(\not D_{\mathrm{h}}^{*}\right)=\left\{\psi \in \mathrm{L}^{2}(\not \Delta \mathcal{N}):\left|\left\langle\psi, \not D_{\mathrm{h}} \varphi\right\rangle\right| \lesssim\|\varphi\|, \varphi \in \mathrm{C}_{\mathrm{c}}^{\infty}(\not \mathcal{N})\right\} .
$$

The first reduction we make is to note that $\mathcal{D}_{\mathrm{c}}\left(\not_{\mathrm{h}}^{*}\right)=\left\{u \in \mathcal{D}\left(\not_{\mathrm{h}}^{*}\right):\right.$ spt $u$ compact $\}$ is dense in $\mathcal{D}\left(\not_{\mathrm{h}}^{*}\right)$. This is a direct consequence of the fact that we are able to find a $C$-close smooth metric $\mathrm{h}^{\prime}$, which is complete since $\mathrm{h}$ is complete, and for this metric $\mathrm{h}^{\prime}$, there exists a sequence of smooth functions $\rho_{k}: \mathcal{N} \rightarrow[0,1]$ with spt $\rho_{k}$ compact, with $\rho_{k} \rightarrow 1$ pointwise, and $\left|\mathrm{d} \rho_{k}\right|_{\mathrm{h}^{\prime}} \leq C^{-1} 1 / k$ for almost-every $x \in \mathcal{N}$ (and hence $\left|\mathrm{d} \rho_{k}\right|_{\mathrm{h}} \leq 1 / k$ for almost-every $\left.x \in \mathcal{N}\right)$. See Proposition 2.3 in [6] or Proposition 1.3.5 in [15] for the existence of such a sequence. The aforementioned density is then simply a consequence of noting the formula $\not_{\mathrm{h}}^{*}(f \varphi)=f \not_{\mathrm{h}}^{*}(\varphi)+(\mathrm{d} f) \cdot \varphi$, for $f \in \mathrm{C}_{\mathrm{c}}^{\infty}(\mathcal{N})$ and $\varphi \in \mathcal{D}\left(\nsupseteq_{\mathrm{h}}^{*}\right)$.

Next, for $\psi \in \mathcal{D}_{\mathrm{c}}\left(\not_{\mathrm{h}}^{*}\right) \cap \mathrm{W}^{1,2}(\Delta \mathcal{N})$, we can write $\psi=\sum_{j=1}^{N} \psi_{j}$ where $\psi_{j}=\eta_{j} \psi$, where $\eta_{j}$ is a finite partition of unity and spt $\eta_{j}$ is contained in a coordinate patch. On obtaining a sequence $\psi^{\delta} \in \mathrm{C}_{\mathrm{c}}^{\infty}(\Delta \mathcal{N})$ by obtaining a mollification $\eta_{j}^{\delta}$ of $\eta_{j}$ inside each coordinate patch, using the fact that $\psi \in \mathrm{W}^{1,2}(\Delta \mathcal{N})$ so that $\left\|\not_{\mathrm{h}}^{*} \psi\right\|=\left\|\not_{\mathrm{h}} \psi\right\| \lesssim$ $\|\nabla \psi\|$, we have that $\psi^{\delta} \rightarrow \psi$ in $\|\cdot\|_{\not_{\mathrm{h}}^{*}}$.

The proof is then complete if we show that whenever $\psi \in \mathcal{D}_{\mathrm{c}}\left(\not_{\mathrm{h}}^{*}\right)$, we have that $\psi \in \mathrm{W}^{1,2}(\boldsymbol{\Delta} \mathcal{N})$. By the compactness of spt $\psi$, we assume without the loss of generality that spt $\psi$ is contained in a coordinate patch corresponding to a ball $B$. Thus assume that for every $\varphi \in \mathrm{C}_{\mathrm{c}}^{\infty}(\not \mathcal{N}),\left|\left\langle\psi, D_{\mathrm{h}} \varphi\right\rangle\right| \lesssim\|\varphi\|$. In particular, this holds when spt $\varphi \subset B$, so let us further assume that. Then, note that

$$
\left\langle\psi, \not D_{\mathrm{h}} \varphi\right\rangle=\int_{B}\left\langle\psi, e^{i} \cdot\left(\partial_{e_{i}} \varphi^{\alpha}\right) \phi_{\alpha}\right\rangle_{*} d \mu_{\mathrm{h}}+\int_{B}\left\langle\psi, e^{i} \cdot \frac{1}{2} \omega^{2}\left(e_{i}\right) \cdot \varphi\right\rangle_{*} d \mu_{\mathrm{h}},
$$


and since $\omega^{2} \in \mathrm{L}^{\infty}(B)$ since $\mathrm{h}$ is locally Lipschitz, we obtain that

$$
\left|\int_{B}\left\langle\psi, e^{i} \cdot\left(\partial_{e_{i}} \varphi^{\alpha}\right) \phi_{\alpha}\right\rangle_{*} d \mu_{\mathrm{h}}\right| \lesssim\|\varphi\| .
$$

Moreover, letting $\mathscr{L}$ denote the Lebesgue measure, we have that $d \mu_{\mathrm{h}}=\theta d \mathscr{L}$, where $\theta=\sqrt{\operatorname{deth}}$ is Lipschitz inside $B$ since h is locally Lipschitz. Thus

$$
\left(\partial_{e_{i}} \varphi^{\alpha}\right) \theta=\partial_{e_{i}}\left(\theta \varphi^{\alpha}\right)-\left(\partial_{e_{i}} \theta\right) \varphi^{\alpha}
$$

and since $\left(\partial_{e_{i}} \theta\right) \in \mathrm{L}^{\infty}(B)$, we further obtain that

$$
\left|\int_{B}\left\langle\psi, e^{i} \cdot \partial_{e_{i}}\left(\theta \varphi^{\alpha}\right) \phi_{\alpha}\right\rangle_{*} d \mathscr{L}\right| \lesssim\|\theta \varphi\|_{\mathrm{L}^{2}(B, \mathscr{L})} .
$$

Now, note that $e^{i} \cdot \phi_{\alpha}=\eta\left(e^{i}\right) \phi_{\alpha}$, which is a constant expression inside $B$. Identifying $B$ with $\chi(B)$ where $\chi: B \rightarrow \mathbb{R}^{n}$ is the coordinate map,

$$
\widehat{\left(e^{i} \cdot f\right)}=e^{i} \cdot \widehat{f}
$$

for $f \in \mathrm{L}^{2}\left(\Delta \mathbb{R}^{n}\right)$, where $\widehat{f}$ is the Fourier Transform of $f$. On extending $\psi$ by zero to all of $\mathbb{R}^{n}$, we obtain that for any $\varphi \in \mathrm{C}_{\mathrm{c}}^{\infty}\left(\mathbb{R}^{n}, \Delta \mathbb{R}^{n}\right)$,

$$
\left|\left\langle\psi, \partial_{e_{i}}\left(\theta \varphi^{\alpha}\right) e^{i} \cdot \phi_{\alpha}\right\rangle_{\mathrm{L}^{2}\left(\Delta \mathbb{R}^{n}\right)}\right| \lesssim\|\theta \varphi\|_{\mathrm{L}^{2}\left(\Delta \mathbb{R}^{n}\right)} .
$$

Then, by Parseval's identity, we have that

$$
\left\langle\psi, \partial_{e_{i}}\left(\theta \varphi^{\alpha}\right) e^{i} \cdot \phi_{\alpha}\right\rangle_{\mathrm{L}^{2}\left(\Delta \mathbb{R}^{n}\right)}=\left\langle\widehat{\psi}, e^{i} \cdot \xi_{i} \widehat{\theta \varphi}\right\rangle_{\mathrm{L}^{2}\left(\Delta \mathbb{R}^{n}\right)}
$$

That is,

$$
\left|\langle\widehat{\psi}, \xi \cdot \widehat{\theta \varphi}\rangle_{\mathrm{L}^{2}\left(\Delta \mathbb{R}^{n}\right)}\right| \lesssim\|\theta \varphi\|_{\mathrm{L}^{2}\left(\Delta \mathbb{R}^{n}\right)}
$$

where $\xi=\xi_{i} e^{i}$ and for all $\varphi \in \mathrm{C}_{\mathrm{c}}^{\infty}\left(\Delta \mathbb{R}^{n}\right)$. Since $\theta \varphi$ is dense in $\mathrm{L}^{2}\left(\Delta \mathbb{R}^{n}\right)$, we have that $\xi \cdot \widehat{\psi} \in \mathrm{L}^{2}\left(\Delta \mathbb{R}^{n}\right)$ (since vectors act skew-adjointly on spinors) which implies that $\psi \in \mathrm{W}^{1,2}\left(\Delta \mathbb{R}^{n}\right)$. On recalling that spt $\psi \subset B$ and that $\omega^{2} \in \mathrm{L}^{\infty}\left(\Omega^{1} \mathcal{M} \otimes \Delta \mathcal{M}\right)$, we have that $\psi \in \mathrm{W}^{1,2}(B, \Delta \mathcal{N})$.

To characterise the domains of the operators $\not_{\mathrm{g}}$ and $\not_{\mathrm{h}}$ as $\mathrm{W}^{1,2}$, we first note the following lemma.

Lemma 3.5. On the manifold $(\mathcal{M}, \mathrm{g})$, there exists a sequence of points $x_{i}$ and a smooth partition of unity $\left\{\eta_{i}\right\}$ uniformly locally finite and subordinate to $\left\{B\left(x_{i}, r_{H}\right)\right\}$ satisfying $\left.\sum_{i}\left|\nabla^{j} \eta_{i}\right|\right) \leq C_{H}$ for $j=0, \ldots, 3$. Moreover, there exists $M>0$ such that $1 \leq M \sum_{i} \eta_{i}^{2}$.

Proof. The proof of this lemma, except for the estimate on the sum of squares of the partition of unity, is included in the proof of Proposition 3.2 in [17]. This is due to the completeness of $g$ and (i) and (ii). We prove the remaining estimate, by noting that by the uniformly locally finite property, there exists a constant $M$ such that for each $x \in \mathcal{M}, 1=\sum_{k=1}^{M} \eta_{i_{k}}(x)$. Moreover, by Cauchy-Schwarz inequality,

$$
1=\left(\sum_{k=1}^{M} \eta_{i_{k}}(x)\right)^{2} \leq\left(\sum_{k=1}^{M} \eta_{i_{k}}(x)^{2}\right)\left(\sum_{k=1}^{M} 1^{2}\right)=M \sum_{i} \eta_{i}^{2}(x) .
$$


With this, the following proposition becomes immediate.

Proposition 3.6. We have $\mathcal{D}\left(\not D_{\mathrm{h}}\right)=\mathrm{W}^{1,2}(\not \mathcal{N})$ with $\left\|\not D_{\mathrm{h}} \varphi\right\|^{2}+\|\varphi\|^{2} \simeq\|\nabla \varphi\|^{2}+$ $\|\varphi\|^{2}$ whenever $\varphi \in \mathrm{W}^{1,2}(\Delta \mathcal{N})$. A similar conclusion holds for $\not_{\mathrm{g}}$.

Proof. By Proposition 3.4, it suffices to demonstrate the estimate $\left\|\not_{\mathrm{h}} \psi\right\|+\|\psi\| \simeq$ $\|\nabla \psi\|+\|\psi\|$ for $\psi \in \mathrm{C}_{\mathrm{c}}^{\infty}(\Delta \mathcal{N})$. From the definition of the operator $\not_{\mathrm{h}}$, we obtain

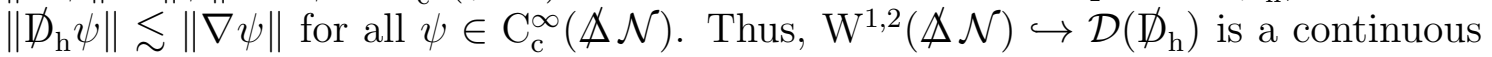
embedding.

Let the partition of unity given by Lemma 3.5 for the metric g be denoted by $\left\{\eta_{i}^{\mathrm{g}}\right\}$. Define $\eta_{i}=\zeta^{*} \eta_{i}^{\mathrm{g}}=\left(\eta_{i}^{\mathrm{g}} \circ \zeta^{-1}\right)$. Now, $\nabla \eta_{i}=\mathrm{d}^{\mathcal{M}} \eta_{i}$ and by the fact that pullback commutes with the exterior derivative, we have that $\mathrm{d}^{\mathcal{N}} \eta_{i}=\mathrm{d}^{\mathcal{N}} \zeta^{*} \eta_{i}^{\mathrm{g}}=\zeta^{*} \mathrm{~d} \mathcal{M} \eta_{i}^{\mathrm{g}}$. Thus, $\sum_{i}\left|\mathrm{~d}^{\mathcal{N}} \eta_{i}\right| \leq C C_{H}$, since $\mathrm{g}$ and $\mathrm{h}$ are $C$-close.

Fix $\psi \in \mathrm{C}_{\mathrm{c}}^{\infty}(\not \mathcal{N})$ so we can write $\psi=\sum_{i=1}^{N} \eta_{i} \psi$. By Fourier theory, we obtain a constant $C^{\prime}>0$ such that $\left\|\nabla\left(\eta_{i} \psi\right)\right\|^{2} \leq C^{\prime}\left(\left\|\not D_{\mathrm{h}}\left(\eta_{i} \psi\right)\right\|^{2}+\left\|\eta_{i} \psi\right\|^{2}\right)$ since spt $\eta_{i} \subset B\left(x_{i}, r_{H}\right)$, which corresponds to a chart for which the metric $\mathrm{g}$ is uniformly comparable to the pullback Euclidean metric.

Moreover, note that since $\nabla$ is a derivation, $\left|\eta_{i} \nabla \psi\right|^{2} \lesssim\left|\mathrm{d}^{\mathcal{N}} \eta_{i}\right|^{2}|\psi|^{2}+\left|\nabla\left(\eta_{i} \varphi\right)\right|^{2}$, and since $|\nabla \psi|^{2} \leq M \sum_{i} \eta_{i}^{2}|\nabla \psi|^{2}$ pointwise by Lemma 3.5,

$$
\begin{array}{r}
\|\nabla \psi\|^{2} \leq M \int \sum_{i}\left|\eta_{i} \nabla \psi\right|^{2} d \mu \lesssim \int \sum_{i}\left|\mathrm{~d}^{\mathcal{N}} \psi\right|^{2}|\psi|^{2} d \mu+\sum_{i} \int\left|\nabla\left(\eta_{i} \psi\right)\right|^{2} d \mu \\
\lesssim\|\psi\|^{2}+\sum_{i} \int\left|\not \emptyset_{\mathrm{h}}\left(\eta_{i} \psi\right)\right|^{2} d \mu .
\end{array}
$$

But by the definition of $\not_{\mathrm{h}}$, we have that

$$
\left|\not D_{\mathrm{h}}\left(\eta_{i} \psi\right)\right|^{2} \lesssim\left|\mathrm{d}^{\mathcal{N}} \eta_{i}\right|^{2}|\psi|^{2}+\eta_{i}^{2}\left|\not D_{\mathrm{h}} \psi\right|^{2}
$$

Integrating this estimate and on combining it with the previous estimates proves the claim. The argument for $\mathrm{g}$ is similar.

Remark 3.7. Typically, the estimate $\left\|\not_{\mathrm{h}} \psi\right\|^{2}+\|\psi\|^{2} \simeq\|\nabla \psi\|^{2}+\|\psi\|^{2}$ is obtained via the Bochner-Lichnerowicz-Schrödinger-Weitzenböck identity:

$$
\not D_{\mathrm{h}}^{2} \psi=-\operatorname{tr} \nabla^{2} \psi+\frac{1}{4} \mathcal{R}_{S}^{\mathrm{h}} \psi
$$

where $\mathcal{R}_{S}^{\mathrm{h}}$ is the scalar curvature of $\mathrm{h}$. This would force $\mathrm{h}$ to be at least $\mathrm{C}^{1,1}$ and we would need to assume that $\mathcal{R}_{S}^{\mathrm{h}} \geq \gamma$ almost-everywhere for some $\gamma \in \mathbb{R}$. However, the fact that $\mathrm{h}$ is $C$-close to the smooth metric $\mathrm{g}$ with stronger curvature bounds allow us to work in the setting where $\mathrm{h}$ is only $\mathrm{C}^{0,1}$. 
3.2. Pullback of Lebesgue and Sobolev spaces of spinors. In this section, we demonstrate that the unitary map $\forall$ as defined before Theorem 3.1 induces maps between $\mathrm{L}^{p}$ spaces and Sobolev spaces.

For the remainder of this section, let us write

$$
\mathrm{B}=\left(P_{\mathrm{g}} P\right)^{-\frac{1}{2}}, \quad \text { and } \quad \theta=\operatorname{det} \mathrm{B}
$$

so that $\mathrm{g}\left(\mathrm{B}^{-1} u, \mathrm{~B}^{-1} v\right)=\tilde{\mathrm{g}}(u, v)$ and $d \mu_{\mathrm{g}}=\theta d \mu_{\tilde{\mathrm{g}}}$.

Proposition 3.8. The isometry $\mathrm{U}:(\mathrm{T} \mathcal{M}, \mathrm{g}) \rightarrow(\mathrm{TN}, \mathrm{h})$ is of class $\mathrm{C}^{0,1}$ and the induced $\forall: \triangle \triangle \mathcal{M} \rightarrow \triangle \Delta \mathcal{M}$ itself induces a bounded invertible map $\forall: \mathrm{L}^{p}(\Delta \mathcal{M}) \rightarrow$ $\mathrm{L}^{p}(\not \mathcal{N})$ for all $p \in[1, \infty]$ satisfying

$$
\|\forall u\|_{L^{p}(\Delta \mathcal{N})} \simeq\|u\|_{L^{p}(\Delta \mathcal{M})}
$$

In what is to follow, let us fix some notation. As noted in the proof of Theorem 3.1, the assumptions we make yields: uniform constants $r_{H}, C>0$ such that at each $x \in \mathcal{M}$, the ball $B\left(x, r_{H}\right)$ is contractible and inside $B\left(x, r_{H}\right)$, we have orthonormal frames $\left\{e_{i}\right\}$ for $\mathrm{T} \mathcal{M}$ and $\left\{\phi_{\alpha}\right\}$ for $\Delta \mathcal{M}$ so that

$$
\left\|e_{i}\right\|_{\mathrm{C}^{2}\left(B\left(x, r_{H}\right)\right)} \leq C \quad \text { and } \quad\left\|\phi_{\alpha}\right\|_{\mathrm{C}^{2}\left(B\left(x, r_{H}\right)\right)} \leq C .
$$

Let the induced orthonormal frame for $\mathrm{T} \mathcal{N}$ and $\Delta \mathcal{N}$ inside $\zeta\left(B\left(x, r_{H}\right)\right)$ respectively

$$
\left\{\tilde{e}_{i}=\mathrm{U} e_{i}\right\} \quad \text { and } \quad\left\{\phi_{\alpha}=\emptyset \phi_{\alpha}\right\} .
$$

Throughout, by $\Omega$ we mean such a ball $B\left(x, r_{H}\right)$.

Lemma 3.9. We have $\omega_{b}^{a}\left(e_{j}\right)=\mathrm{g}\left(\nabla_{e_{j}} e_{b}, e_{a}\right)$ and

$$
2 \mathrm{~g}\left(\nabla_{e_{j}} e_{b}, e_{a}\right)=\mathrm{g}\left(\left[e_{a}, e_{b}\right], e_{j}\right)+\mathrm{g}\left(\left[e_{j}, e_{a}\right], e_{b}\right)-\mathrm{g}\left(\left[e_{b}, e_{j}\right], e_{a}\right)
$$

almost-everywhere inside $\Omega$. Similarly conclusion holds for $\tilde{\omega}_{b}^{a}\left(\tilde{e}_{i}\right)$ with respect to the metric h. Moreover: $\mathrm{h}([\mathrm{U} u, \mathrm{U} v], \mathrm{U} w)=\mathrm{g}\left([\mathrm{B} u, \mathrm{~B} v], \mathrm{B}^{-1} w\right)$.

Proof. We note that $\omega_{b}^{a}\left(e_{j}\right)=w_{j b}^{a}=e^{a}\left(\nabla_{e_{j}} e_{b}\right)=\mathrm{g}\left(\nabla_{e_{j}} e_{b}, e_{a}\right)$ by (3.1). The expression for $2 \mathrm{~g}\left(\nabla_{e_{j}} e_{b}, e_{a}\right)$ is well known. Since $P=\zeta_{*}$, we have $[P u, P v]=P[u, v]$ and on recalling (3.8), we obtain

$$
\begin{aligned}
\mathrm{h}([\mathrm{U} u, \mathrm{U} v], \mathrm{U} w) & =\mathrm{h}([P \mathrm{~B} u, P \mathrm{~B} v], P \mathrm{~B} w)=\mathrm{h}(P[\mathrm{~B} u, \mathrm{~B} v], P \mathrm{~B} w) \\
& =\tilde{\mathrm{g}}([\mathrm{B} u, \mathrm{~B} v], \mathrm{B} w)=\mathrm{g}\left(\mathrm{B}^{-1}[\mathrm{~B} u, \mathrm{~B} v], w\right)=\mathrm{g}\left([\mathrm{B} u, \mathrm{~B} v], \mathrm{B}^{-1} w\right) .
\end{aligned}
$$

The following lemma allow us to relate derivatives of the metric $\tilde{g}=\zeta^{*} \mathrm{~h}$ to the coefficients of the tensorfield $\mathrm{B}$. We note that this lemma can also be obtained via a functional calculus argument. Inside $\Omega$, we write $\mathrm{B}=\left(\beta_{j}^{i}\right)$ and $\mathrm{B}^{-1}=\left(\bar{\beta}_{j}^{i}\right)$.

Lemma 3.10. Then, there is a constant $C_{2}>0$ independent of $\Omega$ such that such that $\left|\partial_{e_{t}} \beta_{j}^{i}\right| \leq C_{2}$ and $\left|\partial_{e_{t}} \bar{\beta}_{j}^{i}\right| \leq C_{2}$ 
Proof. First note that we have $\left|\partial_{e_{t}} \tilde{\mathrm{g}}\left(e_{i}, e_{j}\right)\right| \lesssim 1$ inside $\Omega$, since in this frame,

$$
\nabla^{\mathrm{g}}\left(\zeta^{*} \mathrm{~h}\right)=\left(\partial_{e_{t}} \tilde{\mathrm{g}}_{i j}\right) e^{t} \otimes e^{i} \otimes e^{j}+\tilde{\mathrm{g}}_{i j} e^{t} \otimes \nabla_{e_{t}}\left(e^{i} \otimes e^{j}\right),
$$

and by assumption (iii) of Theorem 3.1, we have that $\left|\nabla^{\mathrm{g}}\left(\zeta^{*} \mathrm{~h}\right)\right| \lesssim 1$ as well as $\left|\nabla_{e_{t}}\left(e^{i} \otimes e^{j}\right)\right| \lesssim 1$. Now, $e_{t} \bar{\beta}_{s}^{r}=-\left(e_{t} \beta_{p}^{q}\right) \bar{\beta}_{q}^{r} \bar{\beta}_{s}^{p}$ and so it suffices to simply bound $\left|e_{t} \beta_{j}^{i}\right| \lesssim 1$. We first note that

$$
e_{t} \tilde{\mathrm{g}}_{r s}=e_{t} \mathrm{~g}\left(\mathrm{~B} e_{r}, \mathrm{~B} e_{s}\right)=e_{t} \mathrm{~g}\left(\mathrm{~B}^{2} e_{r}, e_{s}\right)=e_{t}\left(\mathrm{~B}^{2}\right)_{r s},
$$

where $\mathrm{B}^{2}=\left(\left(\mathrm{B}^{2}\right)_{r s}\right)$ as a matrix. Thus, we obtain $\left|\mathrm{e}_{t} \beta_{s}^{r}\right| \lesssim 1$ if we are able to prove $\left|\mathrm{e}_{t} \mathrm{~B}\right| \lesssim\left|e_{t} \mathrm{~B}^{2}\right|$. Now, by the product rule, note that we obtain $e_{t} \mathrm{~B}^{2}=\mathrm{B}\left(e_{t} \mathrm{~B}\right)+$ $\left(e_{t} \mathrm{~B}\right) \mathrm{B}$, and that, for a vector $u \in \mathrm{T}_{x} \mathcal{M}$ with $|u|=1$,

$$
\begin{array}{r}
\mathrm{g}\left(e_{t} \mathrm{~B}^{2} u, u\right)=\mathrm{g}\left(\left(\mathrm{B} e_{t} \mathrm{~B}\right) u, u\right)+\mathrm{g}\left(\left(e_{t} \mathrm{~B}\right) \mathrm{B} u, u\right) \\
=\mathrm{g}\left(e_{t} \mathrm{~B} u, \mathrm{~B} u\right)+\mathrm{g}\left(\mathrm{B} u, e_{t} \mathrm{~B}\right)=2 \mathrm{~g}\left(\mathrm{~B}\left(e_{t} \mathrm{~B}\right) u, u\right)
\end{array}
$$

since $\mathrm{B}$ is real-symmetric, as is $e_{t} \mathrm{~B}$. This proves that the numerical radius $\operatorname{nrad}\left(e_{t} \mathrm{~B}^{2}\right)=$ $2 \operatorname{nrad}\left(\mathrm{B}\left(e_{t} \mathrm{~B}\right)\right.$. Moreover, note that $\operatorname{nrad}(\cdot)$ is a norm, and since any two norms on a finite dimensional vector space are equivalent, and by the $C$-closeness of $\mathrm{g}$ and $\tilde{\mathrm{g}}$ we have that $|\mathrm{B} u| \geq C^{-1}|u|,\left|e_{t} \mathrm{~B}^{2}\right| \simeq \operatorname{nrad}\left(e_{t} \mathrm{~B}^{2}\right)=2 \operatorname{nrad}\left(\mathrm{B}\left(e_{t} \mathrm{~B}\right)\right) \simeq\left|\mathrm{B}\left(e_{t} \mathrm{~B}\right)\right| \geq$ $C^{-1}\left|e_{t} \mathrm{~B}\right|$.

With the aid of these lemmas, we obtain the following boundedness of $\psi$ between Sobolev spaces.

Proposition 3.11. The space $\forall \mathrm{W}^{1,2}(\not \mathcal{M})=\mathrm{W}^{1,2}(\not \mathcal{N})$ with $\|\mathrm{W} \psi\|+\left\|\nabla^{\mathrm{h}}(\mathrm{W} \psi)\right\| \simeq$ $\|\psi\|+\left\|\nabla^{\mathrm{g}} \psi\right\|$. In fact, the pointwise estimate $|\downarrow \psi|+\left|\nabla^{\mathrm{h}}(W \psi)\right| \simeq|\psi|+\left|\nabla^{\mathrm{g}} \psi\right|$ holds almost-everywhere.

Proof. Note that the assumptions (i)-(iii) in Theorem 3.1 imply an open covering $\left\{\Omega_{p}=B\left(p, r_{H}\right)\right\}$ of $\mathcal{M}$ satisfying $\left|\nabla^{\mathrm{g}} e_{p, i}\right| \leq C$ and $\left|\partial_{e_{p, k}} \tilde{\mathrm{g}}\left(e_{p, i}, e_{p, j}\right)\right| \lesssim C$, where $\left\{e_{p, i}\right\}$ is the frame inside $\Omega_{p}$. So, fix $p$ and let $\psi \in \Gamma(\Delta \mathcal{M})$ be differentiable at $x \in \Omega_{p}$ and note that at $x$,

$$
\left|\nabla^{\mathrm{h}}(\forall \psi)\right|^{2}=\sum_{j}\left|\nabla_{\tilde{e}_{j}}^{\mathrm{h}}(\forall \psi)\right|^{2}=\sum_{j}\left|\psi^{-1} \nabla_{\tilde{e}_{j}}^{\mathrm{h}}(\forall \psi)\right|^{2} .
$$

Now, note that

$$
\nabla_{\tilde{e}_{j}}^{\mathrm{h}}(\downarrow \psi)=\partial_{\tilde{e}_{j}}\left(\psi^{\alpha} \circ \zeta^{-1}\right) \phi_{\alpha}+\left(\psi^{\alpha} \circ \zeta^{-1}\right) \nabla_{\tilde{e}_{j}}^{\mathrm{h}} \phi_{\alpha},
$$

and that by the chain rule, on noting that $\nabla_{\tilde{e}_{j}}^{\mathrm{h}} \mathscr{\phi}_{\alpha}=\frac{1}{2} \sum_{b<a} \omega_{F}^{2}\left(\tilde{e}_{j}\right) \tilde{e}_{b} \cdot \tilde{e}_{a} \cdot \not_{\alpha}$ from (3.2) and (3.3), we obtain that

$$
\psi^{-1} \nabla_{\tilde{e}_{j}}^{\mathrm{h}}(W \psi)=\partial_{B e_{j}}\left(\psi^{\alpha}\right) \phi_{\alpha}+\psi^{\alpha}\left(\omega_{a}^{b}\left(\tilde{e}_{j}\right) \circ \zeta\right) e_{b} \cdot e_{a} \cdot \phi_{\alpha}
$$

We estimate each term on the right side of the equation.

First, note that by Lemma 3.9,

$$
\omega_{a}^{b}\left(\tilde{e}_{j}\right)=\frac{1}{2}\left(\mathrm{~g}\left(\left[\mathrm{~B} e_{a}, \mathrm{~B} e_{b}\right], \mathrm{B}^{-1} e_{j}\right)+\mathrm{g}\left(\left[\mathrm{B} e_{j}, \mathrm{~B} e_{a}\right], \mathrm{B}^{-1} e_{b}\right)-\mathrm{g}\left(\left[\mathrm{B} e_{b}, \mathrm{~B} e_{j}\right], \mathrm{B}^{-1} e_{a}\right)\right)
$$


and by metric compatibility between $\mathrm{g}$ and $\nabla^{\mathrm{g}}$, we have that

$$
\mathrm{g}\left(\left[\mathrm{B} e_{r}, \mathrm{~B} e_{s}\right], \mathrm{B}^{-1} e_{t}\right)=\mathrm{g}\left(\nabla_{\mathrm{B} e_{r}}^{\mathrm{g}}\left(\mathrm{B} e_{s}\right), \mathrm{B}^{-1} e_{t}\right)-\mathrm{g}\left(\nabla_{\mathrm{B} e_{s}}^{\mathrm{g}}\left(\mathrm{B} e_{r}\right), \mathrm{B}^{-1} e_{t}\right) .
$$

We compute

$$
\nabla_{\mathrm{B}_{r}}^{\mathrm{g}}\left(\mathrm{B} e_{s}\right)=\mathrm{B}_{r}^{j} \nabla_{e_{j}}^{\mathrm{g}}\left(\mathrm{B}_{s}^{k} e_{k}\right)=\mathrm{B}_{r}^{j}\left(\left(e_{j} \mathrm{~B}_{s}^{k}\right) e_{k}+\mathrm{B}_{s}^{k} \nabla_{e_{j}}^{\mathrm{g}} e_{k}\right) .
$$

On combining these calculations using Lemma 3.9, we obtain that

$$
\sum_{j}\left|\psi^{\alpha}\left(\omega_{a}^{b}\left(\tilde{e}_{j}\right) \circ \zeta\right) e_{b} \cdot e_{a} \cdot \phi_{\alpha}\right|^{2} \lesssim|\psi|^{2}
$$

To estimate the remaining term, we note that

$$
\left(\partial_{\mathrm{B} e_{j}} \psi^{\alpha}\right) \phi_{\alpha}=\mathrm{B}_{j}^{k}\left(\partial_{e_{k}} \psi^{\alpha}\right) \phi_{\alpha}=\mathrm{B}_{j}^{k} \nabla_{e_{k}}^{\mathrm{g}} \psi-\mathrm{B}_{j}^{k} \psi^{\alpha} \nabla_{e_{k}}^{\mathrm{g}} \phi_{\alpha} .
$$

But by Lemma 3.9

$$
\left|\nabla_{e_{k}}^{\mathrm{g}} \phi_{\alpha}\right| \leq \frac{1}{2} \sum_{b<a}\left|\omega_{a}^{b}\left(e_{k}\right) e_{b} \cdot e_{a} \cdot \phi_{\alpha}\right| \lesssim \sum_{b<a}\left|\nabla_{e_{k}}^{\mathrm{g}} e_{a}\right|\left|e_{b}\right| \lesssim 1
$$

Therefore,

$$
\sum_{j}\left|\left(\partial_{\mathrm{B} e_{j}} \psi^{\alpha}\right) \phi_{\alpha}\right| \lesssim\left|\nabla^{\mathrm{g}} \psi\right|+|\psi| .
$$

This proves the pointwise estimate, and interchanging the roles of $\mathcal{M}$ and $\mathcal{N}$ proves the reverse estimate.

3.3. The pullback Dirac operator and the structural condition. In this section, we pullback the Dirac operator $\not_{\mathrm{h}}$ to on $\boldsymbol{\Delta} \mathcal{N}$ to an operator $\tilde{\not}$ on $\boldsymbol{\Delta} \mathcal{M}$, and prove $(2.7)$.

Fix an $\Omega=B\left(x, r_{H}\right)$ and let $\psi \in \boldsymbol{\Gamma}(\Delta \mathcal{M})$. For $y \in \Omega$ for which $\nabla \psi(y)$ exists, define

$$
\not D \psi(y)=\not_{\mathrm{g}} \psi(y) \quad \text { and } \tilde{\not D} \psi(y)=\mathrm{U}^{-1}(y) \not_{\mathrm{h}}(W \psi)(y) \text {. }
$$

Recall the map B from (3.8) and since $\mathrm{B} \in \boldsymbol{\Gamma}\left(\mathcal{T}^{(1,1)} \mathcal{M}\right)$, in an orthonormal frame $\left\{e_{i}\right\}$, we have that $\mathrm{B} e_{i}=\beta_{i}^{j} e_{j}$ and $\mathrm{B} e^{j}=\beta_{i}^{j} e^{i}$. Moreover, we note that since $\rho_{M}\left(\mathrm{~g}, \zeta^{*} \mathrm{~h}\right) \leq 1,\left|\delta_{i}^{j}-\beta_{i}^{j}\right| \leq\|\mathrm{I}-\mathrm{B}\|_{\infty} \leq \rho_{M}(\mathrm{~g}, \mathrm{~h})$

First, we examine the structure of the difference $\tilde{D}-\not D$ locally in a frame, the main point being the use of the derivation property in Proposition 3.15, before establishing the global result in Proposition 3.16.

Recall from (3.10) that $\tilde{e}_{i}=\mathrm{U} e_{i}$ and $\phi_{\alpha}=\psi \phi \phi_{\alpha}$. Note that this is the fibre-wise $\psi$ and not the $X$ in $\mathrm{L}^{2}$. We also denote the induced fibrewise Clifford bundle pullback between $\Delta \mathcal{M}$ and $\Delta \mathcal{N}$ by $\mathrm{U}$.

Proposition 3.12. We have

$$
(\not D-\tilde{\not D}) \psi=Z \nabla \psi-\left((\mathrm{I}-\mathrm{B}) e^{i}\right) \cdot \omega_{E}^{2}\left(e_{i}\right) \cdot \psi+e^{i} \cdot\left(\omega_{E}^{2}\left(e_{i}\right)-\mathrm{U}^{-1} \omega_{F}^{2}\left(\tilde{e}_{i}\right)\right) \cdot \psi,
$$

distributionally for $\psi \in \mathrm{W}^{1,2}(\Delta \mathcal{M})$, where $Z \in \mathrm{L}^{\infty}\left(\mathrm{T}^{*} \mathcal{M} \otimes \Delta \mathcal{M}, \Delta \mathcal{M}\right)$ with norm $\|Z\|_{\infty} \lesssim\|\mathrm{I}-\mathrm{B}\|_{\infty}$. 
Proof. If $\psi=\psi^{\alpha} \phi_{\alpha}, \forall \psi=\left(\psi^{\alpha} \circ \zeta^{-1}\right) \phi_{\alpha}$, and so

$$
\not D_{\mathrm{h}} \not \psi=\tilde{e}^{i} \cdot \partial_{\tilde{e}_{i}}\left(\psi^{\alpha} \circ \zeta^{-1}\right) \mathscr{\phi}_{\alpha}+\left(\psi^{\alpha} \circ \zeta^{-1}\right) \tilde{e}^{i} \cdot \nabla_{\tilde{e}_{i}} \phi_{\alpha} \text {. }
$$

Thus, on pulling back this expression to $\triangle \mathcal{M}$ via $\emptyset^{-1}$, and invoking the chain rule to the first sum in this expression, we obtain that

$$
\tilde{\not D} \psi=e^{i} \cdot\left(\partial_{\mathrm{B} e_{i}} \psi^{\alpha}\right) \phi_{\alpha}+\psi^{\alpha} e^{i} \cdot \boldsymbol{U}^{-1} \nabla_{\tilde{e}_{i}} \not_{\alpha} .
$$

Thus, the difference of these operators are given by the expression

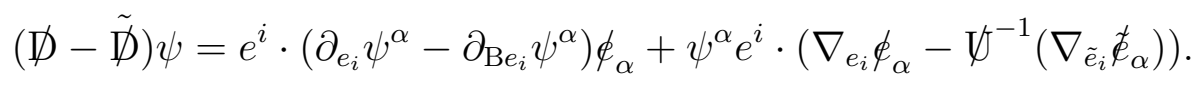

Recalling that $\nabla_{e_{i}} \phi_{\alpha}=\omega_{E}^{2}\left(e_{i}\right) \cdot \phi_{\alpha}$ and that

$$
\bigcup^{-1} \nabla_{\tilde{e}_{i}} \phi_{\alpha}=\psi^{-1}\left(\omega_{F}^{2}\left(\tilde{e}_{i}\right) \cdot \phi_{\alpha}\right)=\mathrm{U}^{-1} \omega_{F}^{2}\left(\tilde{e}_{i}\right) \cdot \psi^{-1} \phi_{\alpha}=\left(\mathrm{U}^{-1} \omega_{F}^{2}\left(\tilde{e}_{i}\right)\right) \cdot \phi_{\alpha} .
$$

The first expression is then given by

$$
\begin{aligned}
e^{i} \cdot\left(\partial_{e_{i}} \psi^{\alpha}-\right. & \left.\partial_{\mathrm{B} e_{i}} \psi^{\alpha}\right) \phi_{\alpha}=\left(\delta_{i}^{j}-\beta_{i}^{j}\right) e^{i} \cdot\left(\partial_{e_{j}} \psi^{\alpha}\right) \phi_{\alpha} \\
& =\left((\mathrm{I}-\mathrm{B}) e^{j}\right) \cdot\left(\partial_{e_{j}} \psi^{\alpha}\right) \phi_{\alpha}=\left((\mathrm{I}-\mathrm{B}) e^{j}\right) \cdot \nabla_{e_{j}} \psi-\psi^{\alpha}(\mathrm{I}-\mathrm{B}) e^{j} \cdot \nabla_{e_{i}} \phi_{\alpha} .
\end{aligned}
$$

Let $\omega=w^{a} \otimes \psi_{a} \in \boldsymbol{\Gamma}\left(\mathrm{T}^{*} \mathcal{M} \otimes \Delta \mathcal{M}\right)$ and define $Z \omega=(\mathrm{I}-\mathrm{B}) w^{a} \cdot \psi_{a}$. This defines a frame invariant expression with

$$
Z \nabla \psi=\left((\mathrm{I}-\mathrm{B}) e^{j}\right) \cdot \nabla_{e_{j}} \psi
$$

and $|Z \omega|=\left|(\mathrm{I}-\mathrm{B}) w^{a} \cdot \psi_{a}\right| \leq\left|(\mathrm{I}-\mathrm{B}) w^{a}\right|\left|\psi_{a}\right| \lesssim\left|w^{a}\right|\left|\psi_{a}\right| \simeq|\omega|$.

As a consequence of this proposition, we will continue to examine remaining terms of the expression $(\not D-\tilde{D}-Z \nabla) \psi$ with the main term being $e^{i} \cdot\left(\omega_{E}^{2}\left(e_{j}\right)-\mathrm{U}^{-1} \omega_{F}^{2}\left(\tilde{e}_{j}\right)\right) \cdot \psi$. Letting $\mathrm{B}^{-1}=\left(\bar{\beta}_{i}^{j}\right)$ in the frame $\left\{e_{i}\right\}$, note that

$$
\begin{aligned}
\left(\omega_{E}^{2}\left(e_{j}\right)-\mathrm{U}^{-1} \omega_{F}^{2}\left(\tilde{e}_{j}\right)\right)=\frac{1}{2} \sum_{b<a}\left(\omega_{a}^{b}\left(e_{i}\right)-\tilde{\omega}_{a}^{b}\left(\tilde{e}_{i}\right) \circ \zeta^{-1}\right) e_{b} \cdot e_{a} \\
=\frac{1}{4} \sum_{b<a}\left\{\left(\mathrm{~g}\left(\left[e_{a}, e_{b}\right], e_{j}\right)+\mathrm{g}\left(\left[e_{j}, e_{a}\right], e_{b}\right)-\mathrm{g}\left(\left[e_{b}, e_{j}\right], e_{a}\right)\right)\right. \\
\left.\quad-\left(\mathrm{h}\left(\left[\tilde{e}_{a}, \tilde{e}_{b}\right], \tilde{e}_{j}\right)+\mathrm{h}\left(\left[\tilde{e}_{j}, \tilde{e}_{a}\right], \tilde{e}_{b}\right)-\mathrm{h}\left(\left[\tilde{e}_{b}, \tilde{e}_{j}\right], \tilde{e}_{a}\right)\right)\right\} e_{b} \cdot e_{a} \\
=\frac{1}{4} \sum_{b<a}\left\{\left(\mathrm{~g}\left(\left[e_{a}, e_{b}\right], e_{j}\right)+\mathrm{g}\left(\left[e_{j}, e_{a}\right], e_{b}\right)-\mathrm{g}\left(\left[e_{b}, e_{j}\right], e_{a}\right)\right)\right. \\
\quad-\left(\mathrm{g}\left(\left[\mathrm{B} e_{a}, \mathrm{~B} e_{b}\right], \mathrm{B}^{-1} e_{j}\right)+\mathrm{g}\left(\left[\mathrm{B} e_{j}, \mathrm{~B} e_{a}\right], \mathrm{B}^{-1} e_{b}\right)\right. \\
\left.\left.\quad-\mathrm{g}\left(\left[\mathrm{B} e_{b}, \mathrm{~B} e_{j}\right], \mathrm{B}^{-1} e_{a}\right)\right)\right\} e_{b} \cdot e_{a}
\end{aligned}
$$

where the last line follows from Lemma 3.9. Hence, it suffices to consider the differences of the form $\mathrm{g}([u, v], w)-\mathrm{g}\left([\mathrm{B} u, \mathrm{~B} v], \mathrm{B}^{-1} w\right)$.

Lemma 3.13. We have

$$
\begin{aligned}
\mathrm{g}\left(\left[e_{i}, e_{j}\right], e_{k}\right)-\mathrm{g}\left(\left[\mathrm{B} e_{i}, \mathrm{~B} e_{j}\right], \mathrm{B}^{-1} e_{k}\right)=\left(\delta_{i}^{a} \delta_{j}^{b} \delta_{k}^{c}\right. & \left.-\beta_{i}^{a} \beta_{j}^{b} \bar{\beta}_{k}^{c}\right) \mathrm{g}\left(\left[e_{a}, e_{b}\right], e_{c}\right) \\
& -\mathrm{g}\left(\left(\partial_{\mathrm{B} e_{i}}\left(\beta_{j}^{a}\right)-\partial_{\mathrm{B} e_{j}}\left(\beta_{i}^{a}\right)\right) e_{a}, \mathrm{~B}^{-1} e_{k}\right)
\end{aligned}
$$


almost-everywhere in $\Omega$.

Proof. Using the derivation property, we obtain that

$$
\begin{aligned}
{\left[\mathrm{B} e_{i}, \mathrm{~B} e_{j}\right] f } & =\partial_{\mathrm{B} e_{i}}\left(\beta_{j}^{b}\right) e_{b}(f)+\beta_{j}^{b} \beta_{i}^{a} e_{a} e_{b}(f)-\partial_{\mathrm{B} e_{j}}\left(\beta_{i}^{a}\right) e_{a}(f)-\beta_{i}^{a} \beta_{j}^{b} e_{b} e_{a}(f) \\
& =\left(\partial_{\mathrm{B} e_{i}}\left(\beta_{j}^{a}\right)-\partial_{\mathrm{B} e_{j}}\left(\beta_{i}^{a}\right)\right) e_{a}(f)+\beta_{i}^{a} \beta_{j}^{b}\left[e_{a}, e_{b}\right] f,
\end{aligned}
$$

where the last equality follows from the fact that $a$ and $b$ are dummy indices, i.e., $\beta_{j}^{b} e_{b}=\beta_{j}^{a} e_{a}$. Therefore,

$$
\begin{aligned}
& \mathrm{g}\left(\left[e_{i}, e_{j}\right], e_{k}\right)-\mathrm{g}\left(\left[\mathrm{B} e_{i}, \mathrm{~B} e_{j}\right], \mathrm{B}^{-1} e_{k}\right)=\mathrm{g}\left(\left[e_{i}, e_{j}\right], e_{k}\right)-\mathrm{g}\left(\left[\mathrm{B} e_{i}, \mathrm{~B} e_{j}\right], \mathrm{B}^{-1} e_{k}\right) \\
& \quad=\mathrm{g}\left(\left[e_{i}, e_{j}\right], e_{k}\right)-\mathrm{g}\left(\beta_{i}^{a} \beta_{j}^{b}\left[e_{a}, e_{b}\right], \bar{\beta}_{k}^{c} e_{c}\right)-\mathrm{g}\left(\left(\partial_{\mathrm{B} e_{i}}\left(\beta_{j}^{a}\right)-\partial_{\mathrm{B} e_{j}}\left(\beta_{i}^{a}\right)\right) e_{a}, \mathrm{~B}^{-1} e_{k}\right) .
\end{aligned}
$$

Then, on noting that $\mathrm{g}\left(\left[e_{i}, e_{j}\right], e_{k}\right)=\delta_{i}^{a} \delta_{j}^{b} \delta_{k}^{c} \mathrm{~g}\left(\left[e_{a}, e_{b}\right], e_{c}\right)$, we obtain the desired conclusion.

With the aid of this, we re-organise the expression (3.12) in the following way:

$$
\begin{aligned}
&\left(\omega_{E}^{2}\left(e_{i}\right)-\right.\left.\mathrm{U}^{-1} \omega_{F}^{2}\left(\tilde{e}_{i}\right)\right) \\
&=\frac{1}{4} \sum_{b<a}\left(\Xi_{a b i}^{q r s}+\Xi_{i a b}^{q r s}-\Xi_{b i a}^{q r s}\right) \mathrm{g}\left(\left[e_{q}, e_{r}\right], e_{s}\right) e_{b} \cdot \mathrm{e}_{a} \\
& \quad+\frac{1}{4} \sum_{b<a}\left(\Upsilon_{a b i}-\Upsilon_{b a i}+\Upsilon_{i a b}-\Upsilon_{a i b}+\Upsilon_{i b a}-\Upsilon_{b i a}\right) e_{b} \cdot e_{a},
\end{aligned}
$$

where $\Xi_{a b c}^{q r s}=\left(\delta_{a}^{q} \delta_{b}^{r} \delta_{c}^{s}-\beta_{a}^{q} \beta_{b}^{r} \bar{\beta}_{c}^{s}\right), \Upsilon_{a b c}=\partial_{\mathrm{B} e_{a}}\left(\beta_{b}^{p}\right) \bar{\beta}_{c}^{q} \delta_{p q}$. We analyse terms of the form $\Upsilon_{r s t} e_{b} \cdot e_{a}$ where $(r, s, t)$ are permutations of $\{a, b, i\}$.

Lemma 3.14. The following holds almost-everywhere in $\Omega$ :

$$
\Upsilon_{a b c}=\operatorname{tr} \nabla^{\mathrm{g}}\left(\Lambda_{a b c}\right)-\epsilon_{b}^{p} \partial_{\mathrm{B} e_{l}}\left(\bar{\beta}_{c}^{q} \theta_{a d}\right) \bar{\beta}_{m}^{l} \delta^{m d}+e_{d}\left(\Lambda_{a b c}\right) w_{m k}^{d} \delta^{m k},
$$

where $\operatorname{tr}$ denotes the trace with respect to the metric $\mathrm{g}$ and where $\epsilon_{b}^{p}=\beta_{b}^{p}-\delta_{b}^{p}$, $\Lambda_{a b c}=\epsilon_{b}^{p} \bar{\beta}_{c}^{q} \delta_{p q} \theta_{a d} e^{d}$ and $\theta_{a d}=\beta_{d}^{a}=\delta_{a k} \beta_{d}^{k}$.

Proof. We compute $\nabla\left(\Lambda_{a b c}\right)$ on letting $v_{a}=\mathrm{B} e_{a}$

$$
\begin{aligned}
\nabla\left(\Lambda_{a b c}\right)= & v^{l} \otimes \nabla_{v_{l}}\left(\epsilon_{b}^{p} \bar{\beta}_{c}^{q} \delta_{p q} \theta_{a d} e^{d}\right) \\
= & \partial_{v_{l}}\left(\epsilon_{b}^{p}\right) \bar{\beta}_{c}^{q} \delta_{p q} \theta_{a d} \bar{\beta}_{m}^{l} e^{m} \otimes e^{d}+\epsilon_{b}^{p} \partial_{v_{l}}\left(\bar{\beta}_{c}^{q} \theta_{a d}\right) \delta_{p q} \bar{\beta}_{m}^{l} e^{m} \otimes e^{d} \\
& +e_{d}\left(\Lambda_{a b c}\right) v^{l} \otimes \nabla_{v_{l}} e^{d} .
\end{aligned}
$$

Now, note that $v^{l} \otimes \nabla_{v_{l}} e^{d}=e^{m} \otimes \nabla_{e_{m}} e^{d}=-w_{m k}^{d} e^{m} \otimes e^{k}$ and hence,

$$
e_{d}\left(\Lambda_{a b c}\right) v^{l} \otimes \nabla_{v_{l}} e^{d}=-e_{d}\left(\Lambda_{a b c}\right) w_{m k}^{d} e^{m} \otimes e^{k} .
$$

Take the trace with respect to $\mathrm{g}$ to get

$$
\begin{aligned}
\operatorname{tr}\left(\partial_{v_{l}}\left(\epsilon_{b}^{p}\right) \bar{\beta}_{c}^{q} \delta_{p q} \theta_{a d} \bar{\beta}_{m}^{l} e^{m} \otimes e^{d}\right) & =\partial_{v_{l}}\left(\epsilon_{b}^{p}\right) \bar{\beta}_{c}^{q} \delta_{p q} \theta_{a d} \bar{\beta}_{m}^{l} \delta^{m d} \\
& =\partial_{v_{l}}\left(\epsilon_{b}^{p}\right) \bar{\beta}_{c}^{q} \delta_{p q} \delta_{a}^{l}=\partial_{v_{a}}\left(\epsilon_{b}^{p}\right) \bar{\beta}_{c}^{q} \delta_{p q}=\Upsilon_{a b c}
\end{aligned}
$$


since $\theta_{a d} \bar{\beta}_{m}^{l} \delta^{m d}=\sum_{m} \theta_{a m} \bar{\beta}_{m}^{l}=\sum_{m} \beta_{m}^{a} \bar{\beta}_{m}^{l}=\delta_{a}^{l}$ by the symmetry of $\beta_{q}^{p}$. This yields the stated identity.

With this, we obtain the following local decomposition.

Proposition 3.15. There are pointwise multiplication operators $X^{\Omega} \in \mathrm{L}^{\infty}(\mathcal{L}(\Delta \Omega))$ and $Y^{\Omega} \in \mathrm{L}^{\infty}\left(\mathcal{L}\left(\mathrm{T}^{*} \Omega \otimes \Delta \Omega, \Delta \Omega\right)\right)$ and $\left.\Lambda^{\Omega} \in \mathrm{L}^{\infty} \cap \operatorname{Lip}\left(\mathcal{L}\left(\Delta \Omega, \mathrm{T}^{*} \Omega \otimes \Delta \Omega\right)\right)\right)$ such that

$$
\begin{aligned}
\operatorname{div}\left(\Lambda^{\Omega} \psi\right)+Y^{\Omega} \nabla \psi+ & X^{\Omega} \psi \\
& =\frac{1}{4} \sum_{b<a}\left(\Upsilon_{a b i}-\Upsilon_{b a i}+\Upsilon_{i a b}-\Upsilon_{a i b}+\Upsilon_{i b a}-\Upsilon_{b i a}\right) e_{b} \Delta e_{a} \cdot \psi
\end{aligned}
$$

holds distributionally for $\psi \in \mathrm{W}^{1,2}(\Delta \mathcal{M})$. Moreover,

$$
\begin{aligned}
& \left\|X^{\Omega}\right\|_{\infty} \lesssim\|\mathrm{I}-\mathrm{B}\|_{\infty}, \quad\left\|Y^{\Omega}\right\|_{\infty} \lesssim\|\mathrm{I}-\mathrm{B}\|_{\infty}, \\
& \left\|\Lambda^{\Omega}\right\|_{\infty} \lesssim\|\mathrm{I}-\mathrm{B}\|_{\infty}, \text { and }\left\|\nabla \Lambda^{\Omega}\right\|_{\infty} \lesssim 1,
\end{aligned}
$$

where the implicit constants in the gradient bound for $\Lambda^{\Omega}$ is independent of $\Omega$.

Proof. By the completeness and smoothness of g along with (i) and (iii) of Theorem 3.1 we have uniform constants $C_{1}, C_{2}>0$ so that $\left|\nabla e_{a}\right| \leq C_{1}$ and $\left|\partial_{e_{c}} \tilde{\mathrm{g}}_{a b}\right| \leq C_{2}$ inside $\Omega$. Let $\Lambda^{\Omega} \psi=\Lambda_{r s t} \otimes\left(e_{b} \cdot e_{a} \cdot \psi\right)=\left(\epsilon_{s}^{p} \bar{\beta}_{t}^{q} \delta_{p q} \delta_{r k} \beta_{d}^{k}\right) e^{d} \otimes\left(e_{b} \cdot e_{a} \cdot \psi\right)$ and note that

$$
\nabla\left(\Lambda_{r s t} \otimes\left(e_{b} \cdot e_{a} \cdot \psi\right)\right)=\nabla\left(\Lambda_{r s t}\right) \otimes\left(e_{b} \cdot e_{a} \cdot \psi\right)+\Lambda_{r s t} \otimes \nabla\left(e_{b} \cdot e_{a} \cdot \psi\right)
$$

where

$$
\nabla\left(e_{b} \cdot e_{a} \cdot \psi\right)=e^{m} \otimes \nabla_{e_{m}}\left(e_{b} \cdot e_{a}\right) \cdot \psi+e^{m} \otimes\left(e_{b} \cdot e_{a}\right) \cdot \nabla_{e_{m}} \psi
$$

Taking traces with respect to $g$, we obtain that

$$
\operatorname{tr} \nabla\left(\Lambda_{r s t}\left(e_{b} \cdot e_{a} \cdot \psi\right)\right)=\left(\operatorname{tr} \nabla\left(\Lambda_{r s t}\right)\right)\left(e_{b} \cdot e_{a} \cdot \psi\right)+\operatorname{tr}\left(\Lambda_{r s t} \otimes \nabla\left(e_{b} \cdot e_{a} \cdot \psi\right)\right) .
$$

Moreover, note that we can write $\Lambda_{r s t}=e_{d}\left(\Lambda_{r s t}\right) e^{d}$ and therefore, we obtain that

$$
\Lambda_{r s t} \otimes \nabla\left(e_{b} \cdot e_{a} \cdot \psi\right)=e_{d}\left(\Lambda_{r s t}\right) e^{d} \otimes e^{m} \otimes \nabla_{e_{m}}\left(e_{b} \cdot e_{a}\right) \cdot \psi+e_{d}\left(\Lambda_{r s t}\right) e^{d} \otimes e^{m} \otimes\left(e_{b} \cdot e_{a}\right) \cdot \nabla_{e_{m}} \psi
$$

so that

$$
\operatorname{tr}\left(\Lambda_{r s t} \otimes \nabla\left(e_{b} \cdot e_{a} \cdot \psi\right)\right)=e_{d}\left(\Lambda_{r s t}\right) \delta^{m d} \nabla_{e_{m}}\left(e_{b} \cdot e_{a}\right) \cdot \psi+e_{d}\left(\Lambda_{r s t}\right) \delta^{d m}\left(e_{b} \cdot e_{a}\right) \cdot \nabla_{e_{m}} \psi .
$$

Define

$$
\begin{aligned}
X_{r s t}^{\Omega} \psi=e_{d}\left(\Lambda_{r s t}\right) \delta^{m d} \nabla_{e_{m}}\left(e_{b} \cdot e_{a}\right) \cdot \psi & \\
& +\left(e_{d}\left(\Lambda_{r s t}\right) w_{m k}^{d} \delta^{m k}-\epsilon_{s}^{p} \partial_{\mathrm{B} e_{l}}\left(\bar{\beta}_{t}^{q} \theta_{r d}\right) \bar{\beta}_{m}^{l} \delta^{m d}\right) e_{b} \cdot e_{a} \cdot \psi,
\end{aligned}
$$

and for $\varphi \in \Gamma\left(\mathrm{T}^{*} \mathcal{M} \otimes \Delta \mathcal{M}\right)$, define

$$
Y_{r s t}^{\Omega} \varphi=Y^{\Omega}\left(\varphi_{a}^{\alpha} e^{a} \otimes \phi_{\alpha}\right)=e_{d}\left(\Lambda_{r s t}\right) \delta^{d a} \varphi_{a}^{\alpha}\left(e_{b} \cdot e_{a}\right) \cdot \phi_{\alpha} .
$$

Estimating with Lemma 3.10, we get $\left\|X_{r s t}^{\Omega}\right\|_{\infty} \lesssim\|\mathrm{I}-\mathrm{B}\|_{\infty},\left\|Y_{r s t}^{\Omega}\right\|_{\infty} \lesssim\|\mathrm{I}-\mathrm{B}\|_{\infty}$, $\left\|\Lambda_{r s t}\right\| \lesssim\|\mathrm{I}-\mathrm{B}\|_{\infty}$ and $\left|\nabla \Lambda_{r s t}^{\Omega}\right| \lesssim 1$. 
Lastly, by taking a sum over permutations over $\{a b c\}$ for the indices $\{r, s, t\}$, the existence of coefficients $X^{\Omega}, Y^{\Omega}$ and $\Lambda^{\Omega}$ as stated in the conclusion is then immediate.

By collating our efforts throughout this section, we obtain the following main result.

Proposition 3.16. We have

$$
\tilde{D} \psi=\not D \psi+A_{1} \nabla \psi+\operatorname{div} A_{2} \psi+A_{3} \psi,
$$

distributionally for $\psi \in \mathrm{W}^{1,2}(\Delta \mathcal{M})$ where the coefficients $A_{1}, A_{2}, A_{3}$ satisfy

$$
\begin{aligned}
& A_{1} \in \mathrm{L}^{\infty}\left(\mathcal{L}\left(\mathrm{T}^{*} \mathcal{M} \otimes \Delta \mathcal{M}, \Delta \mathcal{M}\right)\right), \\
& A_{2} \in \mathrm{L}^{\infty}\left(\mathcal{L}\left(\mathrm{W}^{1,2}(\not \Delta \mathcal{M}), \mathcal{D}(\operatorname{div})\right)\right) \\
& A_{3} \in \mathrm{L}^{\infty}(\mathcal{L}(\Delta \mathcal{M}))
\end{aligned}
$$

with $\left\|A_{1}\right\|_{\infty}+\left\|A_{2}\right\|_{\infty}+\left\|A_{3}\right\|_{\infty} \lesssim\|\mathrm{I}-\mathrm{B}\|_{\infty}$ and $\left\|\nabla A_{2}\right\| \lesssim 1$.

Proof. First, we remark that by the assumptions in Theorem 3.1, exist constants $C_{1}, C_{2}, C_{3}>0$, a covering $\left\{B_{j}\right\}$ which are of fixed radius $r>0$ with orthonormal frames $e_{j, k}$ inside $B_{j}$, and a Lipschitz partition of unity $\left\{\eta_{p}\right\}$ subordinate to $\left\{B_{p}\right\}$ satisfying:

(a) $\left|\nabla e_{j, i}\right| \leq C_{1}$ for all $i$ almost-everywhere on $\overline{B_{p}}$,

(b) $\left|\partial_{e_{j, k}} \tilde{\mathrm{g}}\left(e_{j, i}, e_{j, l}\right)\right| \leq C_{2}$, where $\tilde{\mathrm{g}}=\zeta^{*} \mathrm{~h}$, and

(c) $\left|\nabla \eta_{j}\right| \leq C_{3}$ in $B_{j}$.

Let

$$
W^{B_{j}} \psi=\frac{1}{4} \sum_{b<a}\left(\Xi_{a b i}^{q r s}+\Xi_{i a b}^{q r s}-\Xi_{b i a}^{q r s}\right) \mathrm{g}\left(\left[e_{q}, e_{r}\right], e_{s}\right) e_{b} \cdot \mathrm{e}_{a}-\left((\mathrm{I}-\mathrm{B}) e^{i}\right) \cdot \omega_{E}^{2}\left(e_{i}\right),
$$

and recall the operator $Z$ from Proposition 3.12, $\Lambda^{U}$, and $Y^{U}$ and $X^{U}$ from Proposition 3.15. Inside $B_{j}$, we have the expression

$$
(\tilde{\not D}-\not D) \psi=\sum_{j} \eta_{j} \operatorname{div}\left(\Lambda^{B_{j}} \psi\right)+\left(Z+\sum_{j} \eta^{j} Y^{B_{j}}\right) \nabla \psi+\sum_{j} \eta_{j} X^{B_{j}} \psi+\sum_{j} \eta_{j} W^{B_{j}} \psi
$$

On noting that $\operatorname{div}(\eta \varphi)=\eta \operatorname{div} \varphi+\operatorname{tr}(\nabla \eta \otimes \varphi)$ for $\eta \in \mathrm{C}^{\infty}(\mathcal{M})$ and $\varphi \in \Gamma\left(\mathrm{T}^{*} \mathcal{M} \otimes \mathcal{V}\right)$ differentiable almost-everywhere, we let

$$
\begin{aligned}
& A_{1}=Z+\sum_{j} Y^{B_{j}} \eta_{j}, \\
& A_{2}=\sum_{j} \Lambda^{B_{j}} \eta_{j}, \\
& A_{3}=X^{B_{j}} \eta_{j}+\sum_{j} W^{B_{j}} \eta_{j}-\sum_{j} \operatorname{tr}\left(\left(\nabla \eta_{j}\right) \otimes \psi\right) .
\end{aligned}
$$


It is easy to check that the decomposition of the operator holds almost-everywhere. The conditions (a) and (b) yield that $\left\|A_{1}\right\|+\left\|A_{2}\right\|+\left\|A_{3}\right\| \lesssim\|\mathrm{I}-\mathrm{B}\|_{\infty}$ by Propositions 3.15. Moreover,

$$
\left|\nabla A_{2}\right| \leq \sum_{j}\left|\nabla \eta_{j}\right|\left|\Lambda^{B_{j}}\right|+\sum_{j} \eta_{j}\left|\Lambda^{B_{j}}\right| \lesssim 1,
$$

almost-everywhere uniformly with the constant depending on $C_{1}, C_{2}$ and $C_{3}$.

3.4. Riesz-Weitzenböck formula for Dirac operator. The goal of this subsection is to demonstrate (A9). We begin by noting the following.

Lemma 3.17. The Sobolev spaces satisfy $\mathrm{W}_{0}^{2,2}(\not \mathcal{M})=\mathrm{W}^{2,2}(\not \mathcal{M})$.

Proof. Due to the geometric assumptions (i) and (ii) in Theorem 3.1, the argument to prove the assertion proceeds exactly as Proposition 3.2 in [17], which is a version of this result for functions. The crucial point in the proof is to note that by the derivation property for $\nabla$, for $\eta \in \mathrm{C}^{\infty}(\mathcal{M})$ and $u \in \mathrm{C}^{\infty}(\mathcal{V})$

$$
\left|\nabla^{2}(\eta u)\right| \leq|\eta|\left|\nabla^{2} u\right|+2|\nabla \eta||\nabla u|+\left|\nabla^{2} \eta\right||u|
$$

With this, we obtain the following Riesz-Weizenböck estimate.

Proposition 3.18. There exists $C_{W}>0$ such that $\left\|\nabla^{2} \psi\right\| \leq C_{W}\left(\left\|\not D_{\mathrm{g}}^{2} \psi\right\|+\|\psi\|\right)$ for all $\psi \in \mathcal{D}\left(\not D_{\mathrm{g}}^{2}\right)=\mathrm{W}_{0}^{2,2}(\not \Delta \mathcal{M})=\mathrm{W}^{2,2}(\not \mathcal{M})$.

Proof. Since our metric g is smooth, by Theorem 2.2 in [11], it is well known that $\mathrm{C}_{\mathrm{c}}^{\infty}(\not \mathcal{M})$ is dense (with norm $\|\cdot\|_{\not \not^{2}}$ ) in the domain of $\not_{\mathrm{g}}^{2}$ (and in fact for any positive power $\not_{\mathrm{g}}^{k}$ ). By Lemma 3.17, in order to obtain the conclusion, it suffices to establish

$$
\left\|\nabla^{2} \psi\right\| \lesssim\left\|\not D_{\mathrm{g}}^{2} \psi\right\|+\|\psi\|
$$

for all $\psi \in \mathrm{C}_{\mathrm{c}}^{\infty}(\not \Delta \mathcal{M})$.

First we show that (3.15) holds for $\psi \in \mathrm{C}_{\mathrm{c}}^{\infty}(\Delta \mathcal{M})$ with spt $\psi \subset B\left(x, r_{H}\right)$. To consider just the second-order part of the operator $\not_{\mathrm{g}}^{2}$, we define

$$
\begin{aligned}
L \psi=\not D_{\mathrm{g}}^{2} \psi-e^{i} \cdot e^{j} \cdot\left(\left(e^{j} \psi_{\alpha}\right) \nabla_{e_{i}} \phi_{\alpha}+\left(e_{i} \psi_{\alpha}\right) \nabla_{e_{j}} \phi_{\alpha}+\psi^{\alpha} \nabla_{e_{i}} \nabla_{e_{j}} \phi_{\alpha}\right) & \\
& -e^{i} \cdot \nabla_{e_{i}} e^{j} \cdot \nabla_{e_{j}} \psi
\end{aligned}
$$

Estimating this operator by Plancherel's theorem, we get $\left\|D_{2} \psi\right\|_{\mathrm{L}^{2}\left(B\left(x, r_{H}\right)\right)}^{2} \lesssim\|L \psi\|^{2}+$ $\|\psi\|^{2}$, where $D_{2}=e^{i} \otimes e^{j} \otimes\left(e_{i} e_{j} \psi_{\alpha}\right) \phi_{\alpha}$ is the second-order part of the Hessian. Also,

$$
\begin{aligned}
\|L \psi\|^{2} \lesssim\left\|\not D_{\mathrm{g}}^{2} \psi\right\|^{2}+\max _{\alpha}\left\|\phi_{\alpha}\right\|_{\mathrm{C}^{1}\left(B\left(x, r_{H}\right)\right)}^{2}\|\nabla \psi\|^{2}+\left\|\phi_{\alpha}\right\|_{\mathrm{C}^{2}\left(B\left(x, r_{H}\right)\right)}^{2}\|\psi\|^{2} & \\
& +\max _{j}\left\|e_{j}\right\|_{\mathrm{C}^{1}\left(B\left(x, r_{H}\right)\right)}^{2}\|\nabla \psi\|^{2}
\end{aligned}
$$


As we have noted in (3.9), a consequence of the assumptions (i)-(iii) in Theorem 3.1 is that $\max _{\alpha}\left|\nabla \phi_{\alpha}\right| \lesssim 1$ and $\max _{\alpha}\left|\nabla^{2} \phi_{\alpha}\right| \lesssim 1$ inside $B\left(x, r_{H}\right)$ with constants independent of $B\left(x, r_{H}\right)$. Again, by Plancherel's theorem,

$$
\|\nabla \psi\|^{2} \lesssim\left\|\not D_{\mathrm{g}} \psi\right\|^{2}+\|\psi\|^{2} \lesssim\left\|\not_{\mathrm{g}}^{2} \psi\right\|^{2}+\|\psi\|^{2}
$$

Combining these estimates, we obtain that $\left\|\nabla^{2} \psi\right\|^{2} \lesssim\left\|\not D_{\mathrm{g}}^{2} \psi\right\|^{2}+\|\psi\|^{2}$.

Now, let $\psi \in \mathrm{C}_{\mathrm{c}}^{\infty}(\not \mathcal{M})$ and note by the assumptions we make, on invoking Lemma 3.5, we obtain $C_{H}>0$ such that $\left\{B_{i}=B\left(x_{i}, r_{H}\right)\right\}$ is a cover for $\mathcal{M}$ with $\left\|\mathrm{g}_{i j}\right\|_{\left.\mathrm{C}^{2}\left(B_{i}\right)\right)} \leq$ $C_{H}$ and a smooth partition of unity $\left\{\eta_{i}\right\}$ such that $\sum_{i}\left|\nabla^{j} \eta_{i}\right| \leq C_{H}$ for $j=0, \ldots, 3$. Moreover, this lemma guarantees that there exists $M>0$ such that $1 \leq M \sum_{i} \eta_{i}^{2}$. From the derivation property for $\nabla$, we obtain

$$
\left|\eta_{i} \nabla^{2} \psi\right| \lesssim\left|\nabla^{2} \eta_{i}\right|^{2}|\psi|^{2}+\left|\nabla \eta_{i}\right|^{2}|\nabla \psi|^{2}+\left|\nabla^{2}\left(\eta_{i} \psi\right)\right|^{2}
$$

and we have that

$$
\begin{aligned}
\left\|\nabla^{2} \psi\right\|^{2} \leq & \int M \sum_{i} \eta_{i}^{2}\left|\nabla^{2} \psi\right|^{2} d \mu \\
\leq & M \int \sum_{i}\left|\nabla^{2} \eta_{i}\right|^{2}|\psi|^{2} d \mu+M \int \sum_{i}\left|\nabla \eta_{i}\right|^{2}|\nabla \psi|^{2} d \mu \\
& \quad+M \int \sum_{i}\left|\nabla^{2}\left(\eta_{i} \psi\right)\right|^{2} d \mu \\
\leq\|\psi\|^{2}+ & \quad\|\nabla \psi\|^{2}+\sum_{i}\left\|\nabla^{2}\left(\eta_{i} \psi\right)\right\|^{2} .
\end{aligned}
$$

Now, spt $\left(\eta_{i} \psi\right) \subset \mathrm{B}\left(x_{i}, r_{H}\right)$ and so $\left\|\nabla^{2}\left(\eta_{i} \psi\right)\right\|^{2} \lesssim\left\|\not D_{\mathrm{g}}^{2}\left(\eta_{i} \psi\right)\right\|^{2}+\|\psi\|^{2}$ by what we have just calculated, and so on noting that $\not_{\mathrm{g}}^{2}\left(\eta_{i} \psi\right)=\eta_{i} \not D_{\mathrm{g}}^{2} \psi-2 \nabla_{\left(\operatorname{grad} \eta_{i}\right)} \psi-\left(\Delta \eta_{i}\right) \psi$ by (3.6), where $\operatorname{grad} \eta_{i}=\left(\nabla \eta_{i}\right)^{\sharp}=\mathrm{g}\left(\nabla \eta_{i}, \cdot\right)$, we estimate

$$
\begin{aligned}
\sum_{i}\left\|\nabla^{2}\left(\eta_{i} \psi\right)\right\|^{2} \lesssim \sum_{i} \int \eta_{i}\left|\not D_{\mathrm{g}}^{2} \psi\right|^{2} d \mu+\int \sum_{i}\left|\nabla \eta_{i}\right|^{2}|\psi|^{2} d \mu \\
+\int \sum_{i}\left|\nabla^{2} \eta_{i}\right|^{2}|\psi|^{2} d \mu \\
\lesssim\left\|\not D_{\mathrm{g}}^{2} \psi\right\|^{2}+\|\psi\|^{2} .
\end{aligned}
$$

In Proposition 3.6, we have already shown that $\|\nabla \psi\|^{2} \lesssim\left\|D_{\mathrm{g}} \psi\right\|^{2}+\|\psi\|^{2}$ and hence it suffices to note that

$$
\left\|\not D_{\mathrm{g}} \psi\right\|^{2}=\left\langle\not D_{\mathrm{g}}^{2} \psi, \psi\right\rangle \leq\left\|\not D_{\mathrm{g}}^{2} \psi\right\|\|\psi\| \lesssim\left\|\not D_{\mathrm{g}}^{2} \psi\right\|^{2}+\|\psi\|^{2},
$$

to complete the proof.

\section{Reduction to Quadratic estimates}

The estimates in this section are operator theoretical in their nature and only make use of the structure (2.7) of the perturbation, along with the assumption that $\tilde{D}$ and $\mathrm{D}$ are self-adjoint operators with domains contained in $\mathrm{W}^{1,2}(\mathcal{V})$. We will show how 
to reduce the estimate of $f(\tilde{\mathrm{D}})-f(\mathrm{D})$ in Theorem 2.4 to quadratic estimates. We will see in $\S 5$ that the latter type of estimates allow us to prove the main theorem via harmonic analysis techniques. Throughout this section, we assume the hypothesis of Theorem 2.4.

4.1. Perturbations of resolvents. Since the operators D and D are both selfadjoint, they admit a Borel functional calculus via the spectral theorem as well as a bounded holomorphic functional calculus as outlined in $\S 2.3$.

For $t>0$, let us define operators

$$
\mathrm{P}_{t}=\frac{1}{\mathrm{I}+t^{2} \mathrm{D}^{2}}, \tilde{\mathrm{P}}_{t}=\frac{1}{\mathrm{I}+t^{2} \tilde{\mathrm{D}}^{2}}, \mathrm{Q}_{t}=t \mathrm{DP}_{t}, \quad \text { and } \quad \tilde{\mathrm{Q}}_{t}=t \tilde{\mathrm{D}} \tilde{\mathrm{P}}_{t} .
$$

The fact that $\mathrm{D}$ and $\tilde{\mathrm{D}}$ are self-adjoint gives

$$
\int_{0}^{\infty}\left\|\tilde{\mathrm{Q}}_{t} u\right\|^{2} \frac{d t}{t} \leq \frac{1}{2}\|u\|^{2} \text { and } \int_{0}^{\infty}\left\|\mathrm{Q}_{t} u\right\|^{2} \frac{d t}{t} \leq \frac{1}{2}\|u\|^{2},
$$

and also

$$
\sup _{t}\left\|\mathrm{P}_{t}\right\|, \sup _{t}\left\|\tilde{\mathrm{P}}_{t}\right\|, \sup _{t}\left\|\mathrm{Q}_{t}\right\|, \sup _{t}\left\|\tilde{\mathrm{Q}}_{t}\right\| \leq \frac{1}{2}
$$

Furthermore, we note that the operators $\mathrm{P}_{t}, \mathrm{P}_{t}, \mathrm{Q}_{t}, \tilde{\mathrm{Q}}_{t}$ are self-adjoint.

Moreover, let

$$
\psi(\zeta)=\frac{\zeta}{1+\zeta^{2}} \quad \text { and } \quad \psi_{t}(\zeta)=\psi(t \zeta)
$$

and note that $\mathrm{Q}_{t}=\psi_{t}(\mathrm{D})$ and $\tilde{\mathrm{Q}}_{t}=\psi_{t}(\tilde{\mathrm{D}})$. We establish some operator theoretic facts about $\tilde{Q}_{t}$ and $\mathrm{Q}_{t}$ that will be of use to us later.

Let

$$
\tilde{\mathrm{R}}_{t}=\frac{1}{\mathrm{I}+\mathrm{i} t \tilde{\mathrm{D}}}=-(\mathrm{i} t)^{-1} \mathrm{R}_{\tilde{\mathrm{D}}}\left(-(\mathrm{i} t)^{-1}\right) \quad \text { and } \quad \mathrm{R}_{t}=\frac{1}{\mathrm{I}+\mathrm{i} t \mathrm{D}}=-(\mathrm{i} t)^{-1} \mathrm{R}_{\mathrm{D}}\left(-(\mathrm{i} t)^{-1}\right),
$$

and note that

$$
\tilde{\mathrm{R}}_{t}=\frac{1}{\mathrm{I}+\mathrm{i} t \tilde{\mathrm{D}}}=\frac{1}{\mathrm{I}+\mathrm{i} t \tilde{\mathrm{D}}} \frac{\mathrm{I}-\mathrm{i} t \tilde{\mathrm{D}}}{\mathrm{I}-\mathrm{i} t \tilde{\mathrm{D}}}=\frac{1}{\mathrm{I}+t^{2} \tilde{\mathrm{D}}^{2}}-\mathrm{i} \frac{t \tilde{\mathrm{D}}}{\mathrm{I}+t^{2} \tilde{\mathrm{D}}^{2}}=\tilde{\mathrm{P}}_{t}-\mathrm{i} \tilde{\mathrm{Q}}_{t} .
$$

Similarly, $\mathrm{R}_{t}=\mathrm{P}_{t}-\mathrm{iQ}_{t}$.

Proposition 4.1. The difference of the resolvents satisfies the formula:

$$
\tilde{\mathrm{R}}_{t}-\mathrm{R}_{t}=\tilde{\mathrm{R}}_{t}[\mathrm{i} t(\mathrm{D}-\tilde{\mathrm{D}})] \mathrm{R}_{t} \text {. }
$$

Moreover,

$$
\tilde{\mathrm{Q}}_{t}-\mathrm{Q}_{t}=-\tilde{\mathrm{P}}_{t}[t(\tilde{\mathrm{D}}-\mathrm{D})] \mathrm{P}_{t}-\tilde{\mathrm{Q}}_{t}[t(\tilde{\mathrm{D}}-\mathrm{D})] \mathrm{Q}_{t}
$$

Proof. First, note that:

$$
\tilde{\mathrm{R}}_{t}-\mathrm{R}_{t}=\tilde{\mathrm{R}}_{t}(1+\mathrm{i} t \mathrm{D}) \mathrm{R}_{t}-\tilde{\mathrm{R}}_{t}(1+\mathrm{i} t \tilde{\mathrm{D}}) \mathrm{R}_{t} .
$$


Since by assumption $\mathcal{D}(\tilde{\mathrm{D}})=\mathcal{D}(\mathrm{D})=\mathrm{W}^{1,2}(\mathcal{V})$, we have that $\mathcal{R}\left(\tilde{\mathrm{R}}_{t}\right)=\mathcal{D}(\tilde{\mathrm{D}})$ and hence, $(\mathrm{I}+\mathrm{i} t \tilde{\mathrm{D}}) \mathrm{R}_{t} \in \mathcal{L}(\mathscr{H})$. Thus,

$$
\tilde{\mathrm{R}}_{t}-\mathrm{R}_{t}=\tilde{\mathrm{R}}_{t}[(1+\mathrm{i} t \mathrm{D})-(1+\mathrm{i} t \tilde{\mathrm{D}})] \mathrm{R}_{t}=\tilde{\mathrm{R}}_{t}[\mathrm{i} t(\mathrm{D}-\tilde{\mathrm{D}})] \mathrm{R}_{t} .
$$

Expanding $\tilde{\mathrm{R}}_{t}=\tilde{\mathrm{P}}_{t}-\mathrm{i} \tilde{\mathrm{Q}}_{t}$ as we noted in (4.1), a straightforward calculation yields that

$$
\begin{aligned}
\left(\tilde{\mathrm{P}}_{t}-\mathrm{P}_{t}\right)-\mathrm{i}\left(\tilde{\mathrm{Q}}_{t}-\mathrm{Q}_{t}\right)=\tilde{\mathrm{R}}_{t}-\mathrm{R}_{t}=\tilde{\mathrm{P}}_{t} & {[t(\mathrm{D}-\tilde{\mathrm{D}})] \mathrm{Q}_{t}+\tilde{\mathrm{Q}}_{t}[t(\mathrm{D}-\tilde{\mathrm{D}})] \mathrm{P}_{t} } \\
& +\mathrm{i}\left\{\tilde{\mathrm{P}}_{t}[t(\mathrm{D}-\tilde{\mathrm{D}})] \mathrm{P}_{t}+\tilde{\mathrm{Q}}_{t}[t(\mathrm{D}-\tilde{\mathrm{D}})] \mathrm{Q}_{t}\right\}
\end{aligned}
$$

which shows the expression for $\tilde{\mathrm{Q}}_{t}-\tilde{\mathrm{Q}}_{t}$.

In particular, we see that

$$
\begin{aligned}
& \left\|\left(\tilde{\mathrm{Q}}_{t}-\mathrm{Q}_{t}\right) f\right\| \\
& \leq\left\|\tilde{\mathrm{P}}_{t}\left(t A_{1} \nabla\right) \mathrm{P}_{t} f\right\|+\left\|\tilde{\mathrm{P}}_{t}\left(t \operatorname{div} A_{2}\right) \mathrm{P}_{t} f\right\|+\left\|\tilde{\mathrm{P}}_{t}\left(t A_{3}\right) \mathrm{P}_{t} f\right\| \\
& \quad+\left\|\tilde{\mathrm{Q}}_{t}\left(t A_{1} \nabla\right) \mathrm{Q}_{t} f\right\|+\left\|\tilde{\mathrm{Q}}_{t}\left(t \operatorname{div} A_{2}\right) \mathrm{Q}_{t} f\right\|+\left\|\tilde{\mathrm{Q}}_{t}\left(t A_{3}\right) \mathrm{Q}_{t} f\right\|,
\end{aligned}
$$

Proposition 4.2. We obtain the estimates

$$
\sup _{t \in(0,1]}\left\|\tilde{\mathrm{Q}}_{t}-\mathrm{Q}_{t}\right\| \lesssim\|A\|_{\infty}, \quad \sup _{t \in(0,1]}\left\|\tilde{\mathrm{R}}_{t}-\mathrm{R}_{t}\right\| \lesssim\|A\|_{\infty},
$$

where the implicit constants depend on $\mathrm{C}(\mathcal{M}, \mathcal{V}, \mathrm{D}, \tilde{\mathrm{D}})$.

Proof. First, we bound the terms with $\tilde{\mathrm{P}}_{t}$ and $\mathrm{P}_{t}$. Note that,

$$
\left\|\tilde{\mathrm{P}}_{t}\left(t A_{1} \nabla\right) \mathrm{P}_{t}\right\| \leq\left(\sup _{t \in(0,1]}\left\|\tilde{\mathrm{P}}_{t}\right\|\right)\left\|A_{1}\right\|_{\infty}\left\|t \nabla \mathrm{P}_{t}\right\| .
$$

Moreover, by (2.4),

$$
\left\|t \nabla \mathrm{P}_{t}\right\| \leq C_{\mathrm{D}}\left(\left\|t \mathrm{DP}_{t}\right\|+\left\|t \mathrm{P}_{t}\right\|\right) \leq \mathrm{C}(1+t) .
$$

On combining this with the assumption that $\left\|A_{1}\right\|_{\infty} \leq\|A\|_{\infty}$, we obtain that $\left\|\tilde{\mathrm{P}}_{t}\left(t A_{1} \nabla\right) \mathrm{P}_{t}\right\| \leq \mathrm{C}\|A\|_{\infty}(1+t)$.

Next, we estimate $\left\|\tilde{\mathrm{P}}_{t}\left(t \operatorname{div} A_{2}\right) \mathrm{P}_{t}\right\|$. First, we note that, for $v \in \mathcal{D}(\operatorname{div})$,

$$
\left\|\tilde{\mathrm{P}}_{t}(t \operatorname{div}) v\right\|=\sup _{\|g\|=1}\left|\left\langle\tilde{\mathrm{P}}_{t}(t \operatorname{div}) v, g\right\rangle\right|=\sup _{\|g\|=1}\left|\left\langle v, t \operatorname{div}^{*} \tilde{\mathrm{P}}_{t} g\right\rangle\right| \leq \sup _{\|g\|=1}\|v\|\left\|t \operatorname{div}^{*} \tilde{\mathrm{P}}_{t} g\right\| .
$$

Now, note that $\operatorname{div}^{*}=-\nabla$ and on invoking (2.4),

$$
\left\|t \operatorname{div}^{*} \tilde{\mathrm{P}}_{t} g\right\| \leq \mathrm{C}\left(\left\|t \tilde{\mathrm{D}} \tilde{\mathrm{P}}_{t} g\right\|+\left\|t \tilde{\mathrm{P}}_{t} g\right\|\right) \leq \mathrm{C}(1+t)\|g\| .
$$

Thus, $\left\|\tilde{\mathrm{P}}_{t}(t \operatorname{div}) v\right\| \leq 2 \mathrm{C}\|v\|$ and since $\mathcal{D}($ div $)$ is dense in $\mathrm{L}^{2}\left(\mathrm{~T}^{*} \mathcal{M} \otimes \mathcal{V}\right)$, we obtain that $\tilde{\mathrm{P}}_{t}(t$ div $)$ extends to a bounded operator, uniformly bounded in $t \in(0,1]$. Thus,

$$
\left\|\tilde{\mathrm{P}}_{t}\left(t \operatorname{div} A_{2}\right) \mathrm{P}_{t}\right\| \leq\left\|\tilde{\mathrm{P}}_{t}(t \operatorname{div})\right\|\left\|A_{2}\right\|_{\infty}\left\|\mathrm{P}_{t}\right\| \leq \mathrm{C}\|A\|_{\infty} .
$$

It is immediate that $\left\|\tilde{\mathrm{P}}_{t} A_{3} \mathrm{P}_{t}\right\| \leq\left\|\tilde{\mathrm{P}}_{t}\right\|\left\|A_{3}\right\|_{\infty}\left\|\mathrm{P}_{t}\right\| \leq\|A\|_{\infty}$. 
Similar bounds for $\tilde{\mathrm{Q}}_{t}$ and $\mathrm{Q}_{t}$ in place of $\tilde{\mathrm{P}}_{t}$ and $\mathrm{P}_{t}$ follow by exactly the same arguments noting that $\left\|t \nabla \mathrm{Q}_{t}\right\| \simeq\left\|\mathrm{I}-\mathrm{P}_{t}\right\|$. This shows that $\sup _{t \in(0,1]}\left\|\tilde{\mathrm{Q}}_{t}-\mathrm{Q}_{t}\right\| \lesssim$ $\|A\|_{\infty}$. To show $\sup _{t \in(0,1]}\left\|\tilde{\mathrm{R}}_{t}-\mathrm{R}_{t}\right\| \lesssim\|A\|_{\infty}$, we note that it suffices to simply verify that the previous argument holds for $\tilde{\mathrm{R}}_{t}$ and $\mathrm{R}_{t}$ in place of $\tilde{\mathrm{P}}_{t}$ and $\mathrm{P}_{t}$ due to the formula established in Proposition 4.1.

We note that a similar estimate of $P_{t}$ also hold, but we shall not need that.

4.2. First reduction. Now, let $f \in \operatorname{Hol}^{\infty}\left(\mathrm{S}_{\omega, \sigma}^{o}\right)$, for $\omega \in(0, \pi / 2)$ and $\sigma \in(0, \infty)$. We reduce estimating $\|f(\tilde{\mathrm{D}})-f(\mathrm{D})\|$ to obtaining an appropriate estimate for $\| \tilde{\mathrm{Q}}_{t}-$ $\mathrm{Q}_{t} \|$. To that end, we begin with the following lemma.

Lemma 4.3. The following identities hold:

$$
\mathrm{I}=\tilde{\mathrm{P}}_{1}+2 \int_{0}^{1} \tilde{\mathrm{Q}}_{s}^{2} \frac{d s}{s}=\mathrm{P}_{1}+2 \int_{0}^{1} \mathrm{Q}_{s}^{2} \frac{d s}{s},
$$

where $\tilde{\mathrm{P}}_{1}=\left(\mathrm{I}+\tilde{\mathrm{D}}^{2}\right)^{-1}$ and $\mathrm{P}_{1}=\left(\mathrm{I}+\mathrm{D}^{2}\right)^{-1}$.

Proof. Note that,

$$
\mathrm{I}-\mathrm{P}_{1}=\mathrm{I}-\left(\mathrm{I}+\mathrm{D}^{2}\right)^{-1}=\mathrm{D}^{2}\left(\mathrm{I}+\mathrm{D}^{2}\right)^{-1}
$$

Moreover,

$$
\frac{d}{d s}\left(\frac{s^{2}}{1+s^{2}}\right)=\frac{2 s}{\left(1+s^{2}\right)^{2}}
$$

and by setting $s=t z$, we have that

$$
\int_{0}^{1} \frac{(t z)^{2}}{\left(1+(t z)^{2}\right)^{2}} \frac{d t}{t}=\int_{0}^{z} \frac{s^{2}}{\left(1+s^{2}\right)^{2}} \frac{d s}{s}=\frac{1}{2} \frac{z^{2}}{1+z^{2}} .
$$

Thus, by the functional calculus we obtain that

$$
\mathrm{D}^{2}\left(\mathrm{I}+\mathrm{D}^{2}\right)^{-1} u=2 \int_{0}^{1} \psi_{t}(\mathrm{D})^{2} u \frac{d t}{t} .
$$

The calculation for $\tilde{D}^{2}\left(\mathrm{I}+\tilde{\mathrm{D}}^{2}\right)^{-1}$ is similar.

With the aid of this lemma, we obtain

$$
\begin{aligned}
f(\tilde{\mathrm{D}})-f(\mathrm{D})=\left[\tilde{\mathrm{P}}_{1}+\right. & \left.\left(\mathrm{I}-\tilde{\mathrm{P}}_{1}\right)\right] f(\tilde{\mathrm{D}})\left[\tilde{\mathrm{P}}_{1}+\left(\mathrm{I}-\tilde{\mathrm{P}}_{1}\right)\right] \\
& \quad-\left[\mathrm{P}_{1}+\left(\mathrm{I}-\mathrm{P}_{1}\right)\right] f(\mathrm{D})\left[\mathrm{P}_{1}+\left(\mathrm{I}-\mathrm{P}_{1}\right)\right] \\
=\left[\left(2 \tilde{\mathrm{P}}_{1}-\right.\right. & \left.\left.\tilde{\mathrm{P}}_{1}^{2}\right) f(\tilde{\mathrm{D}})-\left(2 \mathrm{P}_{1}-\mathrm{P}_{1}^{2}\right) f(\mathrm{D})\right] \\
& \quad+4 \int_{0}^{1} \int_{0}^{1}\left[\left(\psi_{s}^{2} f \psi_{t}^{2}\right)(\tilde{\mathrm{D}})-\left(\psi_{s}^{2} f \psi_{t}^{2}\right)(\mathrm{D})\right] \frac{d s}{s} \frac{d t}{t} .
\end{aligned}
$$


Consider the second term on the right. Using the fact that the functional calculus is a homomorphism yields that

$$
\begin{aligned}
\left(\psi_{s}^{2} f \psi_{t}^{2}\right)(\tilde{\mathrm{D}})-\left(\psi_{s}^{2} f \psi_{t}^{2}\right)(\mathrm{D})= & \psi_{s}(\tilde{\mathrm{D}})\left(\psi_{s} f \psi_{t}\right)(\tilde{\mathrm{D}})\left[\psi_{t}(\tilde{\mathrm{D}})-\psi_{t}(\mathrm{D})\right] \\
& +\psi_{s}(\tilde{\mathrm{D}})\left[\left(\psi_{s} f \psi_{t}\right)(\tilde{\mathrm{D}})-\left(\psi_{s} f \psi_{t}\right)(\mathrm{D})\right] \psi_{t}(\mathrm{D}) \\
& +\left[\psi_{s}(\tilde{\mathrm{D}})-\psi_{s}(\mathrm{D})\right]\left(\psi_{s} f \psi_{t}\right)(\mathrm{D}) \psi_{t}(\mathrm{D})
\end{aligned}
$$

Let $\eta(x)=\min \left\{x, \frac{1}{x}\right\}(1+|\log | x||)$. Then, we have the following preliminary estimates for each of the three terms appearing in (4.4).

Lemma 4.4. The following estimates hold:

$$
\begin{aligned}
\left\|\left(\psi_{s} f \psi_{t}\right)(\tilde{\mathrm{D}})\right\| \lesssim\|f\|_{\infty} \eta(s / t), \quad & \left\|\left(\psi_{s} f \psi_{t}\right)(\mathrm{D})\right\| \lesssim\|f\|_{\infty} \eta(s / t), \text { and } \\
& \left\|\left(\psi_{s} f \psi_{t}\right)(\tilde{\mathrm{D}})-\left(\psi_{s} f \psi_{t}\right)(\mathrm{D})\right\| \lesssim\|f\|_{\infty}\|A\|_{\infty} \eta(s / t),
\end{aligned}
$$

where the implicit constants only depend on $\mathrm{C}(\mathcal{M}, \mathcal{V}, \mathrm{D}, \tilde{\mathrm{D}})$.

Proof. The bound for the first two terms follows directly from the norm estimate of the Riesz-Dunford integral (2.1). For the last estimate, we have that, after fixing an appropriate curve $\gamma$,

$$
\begin{aligned}
&\left\|\left(\psi_{s} f \psi_{t}\right)(\tilde{\mathrm{D}})-\left(\psi_{s} f \psi_{t}\right)(\mathrm{D})\right\| \lesssim \oint_{\gamma}\left\|\left(\psi_{s} f \psi_{t}\right)(\zeta)\left(\mathrm{R}_{\tilde{\mathrm{D}}}(\zeta)-\mathrm{R}_{\mathrm{D}}(\zeta)\right)\right\||d \zeta| \\
& \lesssim\|f\|_{\infty} \eta(s / t)\left(\oint_{\gamma}\left\|\psi_{s} f \psi_{t} \psi(\zeta)\right\| \frac{|d \zeta|}{|\zeta|}\right) \sup _{\zeta \in \gamma}\left(\left\|\mathrm{R}_{\tilde{\mathrm{D}}}(\zeta)-\mathrm{R}_{\mathrm{D}}(\zeta)\right\||\zeta|\right) \\
& \lesssim\|f\|_{\infty}\|A\|_{\infty} \eta(s / t),
\end{aligned}
$$

where the penultimate inequality follows from the decay of $\psi_{s} f \psi_{t}$ and from Proposition 4.2.

Proposition 4.5. Suppose that

$$
\int_{0}^{1}\left\|\left(\tilde{\mathrm{Q}}_{t}-\mathrm{Q}_{t}\right) u\right\|^{2} \frac{d t}{t} \leq C_{0}\|A\|_{\infty}^{2}\|u\|^{2}
$$

for all $u \in \mathrm{L}^{2}(\mathcal{V})$. Then,

$$
\|f(\tilde{\mathrm{D}})-f(\mathrm{D})\| \lesssim\|A\|_{\infty}\|f\|_{\infty},
$$

where the implicit constant depends only on $\mathrm{C}(\mathcal{M}, \mathcal{V}, \mathrm{D}, \tilde{\mathrm{D}})$ and $C_{0}$.

Proof. We appeal to (4.3) and first prove that

$$
\left\|\left(2 \tilde{\mathrm{P}}_{1}-\tilde{\mathrm{P}}_{1}^{2}\right) f(\tilde{\mathrm{D}})-\left(2 \mathrm{P}_{1}-\mathrm{P}_{1}^{2}\right) f(\mathrm{D})\right\| \lesssim\|f\|_{\infty}\|A\|_{\infty} .
$$

To that end, define

$$
\varphi(\zeta)=\left(\frac{2}{1+\zeta^{2}}-\frac{1}{\left(1+\zeta^{2}\right)^{2}}\right) f(\zeta)
$$


and note that $\varphi \in \Psi\left(\mathrm{S}_{\omega, \sigma}^{\circ}\right)$. Moreover, by the functional calculus, we have $\left[\left(2 \tilde{\mathrm{P}}_{1}-\right.\right.$ $\left.\left.\tilde{\mathrm{P}}_{1}^{2}\right) f(\tilde{\mathrm{D}})-\left(2 \mathrm{P}_{1}-\mathrm{P}_{1}^{2}\right) f(\tilde{\mathrm{D}})\right]=\varphi(\tilde{\mathrm{D}})-\varphi(\mathrm{D})$. Then, for an appropriate chosen curve $\gamma$

$$
\begin{aligned}
\|\varphi(\tilde{\mathrm{D}}) u-\varphi(\mathrm{D}) u\| & \lesssim\|f\|_{\infty} \oint_{\gamma}|\varphi(\zeta)|\left\|\mathrm{R}_{\tilde{\mathrm{D}}}(\zeta)(\mathrm{D}-\tilde{\mathrm{D}}) \mathrm{R}_{\mathrm{D}}(\zeta) u\right\||d \zeta| \\
& \lesssim\|f\|_{\infty}\|A\|_{\infty}\|u\|\left(\oint_{\gamma}|\varphi(\zeta)|\right) \frac{|d \zeta|}{|\zeta|} \lesssim\|f\|_{\infty}\|A\|_{\infty}\|u\|
\end{aligned}
$$

where the first inequality follows from Proposition 4.2.

Now, to bound the second term of (4.3),we appeal to (4.4). As we have previously noted, $\psi_{t}(\mathrm{D})=\mathrm{Q}_{t}$ and $\psi_{t}(\tilde{\mathrm{D}})=\tilde{\mathrm{Q}}_{t}$, and so,

$$
\begin{aligned}
& \left\|\psi_{s}(\tilde{\mathrm{D}})\left(\psi_{s} f \psi_{t}\right)(\tilde{\mathrm{D}})\left[\psi_{t}(\tilde{\mathrm{D}})-\psi_{t}(\mathrm{D})\right]\right\| \\
& =\sup _{\|u\|=\|v\|=1}\left|\left\langle\psi_{s}(\tilde{\mathrm{D}})\left(\psi_{s} f \psi_{t}\right)(\tilde{\mathrm{D}})\left[\psi_{t}(\tilde{\mathrm{D}})-\psi_{t}(\mathrm{D})\right] u, v\right\rangle\right| \\
& \quad=\sup _{\|u\|=\|v\|=1}\left|\left\langle\left(\psi_{s} f \psi_{t}\right)(\tilde{\mathrm{D}})\left(\tilde{\mathrm{Q}}_{t}-\mathrm{Q}_{t}\right) u, \tilde{\mathrm{Q}}_{s} v\right\rangle\right| .
\end{aligned}
$$

Fix $\|u\|=\|v\|=1$, and we compute

$$
\begin{aligned}
\left|\left\langle\left(\psi_{s} f \psi_{t}\right)(\tilde{\mathrm{D}})\left(\tilde{\mathrm{Q}}_{t}-\mathrm{Q}_{t}\right) u, \tilde{\mathrm{Q}}_{s} v\right\rangle\right| \lesssim\left\|\psi_{s} f \psi_{t}(\tilde{\mathrm{D}})\left(\tilde{\mathrm{Q}}_{t}-\mathrm{Q}_{t}\right) u\right\|\left\|\tilde{\mathrm{Q}}_{s} v\right\| \\
\\
\quad \lesssim\|f\|_{\infty} \eta(s / t)\left\|\left(\tilde{\mathrm{Q}}_{t}-\mathrm{Q}_{t}\right) u\right\|\left\|\tilde{\mathrm{Q}}_{s} v\right\| .
\end{aligned}
$$

Thus,

$$
\begin{aligned}
\int_{0}^{1} \int_{0}^{1} & \left\langle\left(\psi_{s} f \psi_{t}\right)(\tilde{\mathrm{D}})\left(\tilde{\mathrm{Q}}_{t}-\mathrm{Q}_{t}\right) u, \tilde{\mathrm{Q}}_{s} v\right\rangle \mid \frac{d s}{s} \frac{d t}{t} \\
& \lesssim\|f\|_{\infty}\left(\int_{0}^{1}\left(\int_{0}^{1} \eta(s / t)\left\|\left(\tilde{\mathrm{Q}}_{t}-\mathrm{Q}_{t}\right) u\right\|^{2} \frac{d s}{s}\right) \frac{d t}{t}\right)^{\frac{1}{2}} \times \\
& \left(\int_{0}^{1} \int_{0}^{1} \eta(s / t)\left\|\tilde{\mathrm{Q}}_{s} v\right\|^{2} \frac{d s}{s} \frac{d t}{t}\right)^{\frac{1}{2}} \\
& \lesssim\|f\|_{\infty}\left(\int_{0}^{1}\left\|\left(\tilde{\mathrm{Q}}_{t}-\mathrm{Q}_{t}\right) u\right\|^{2} \frac{d t}{t}\right)^{\frac{1}{2}}\left(\int_{0}^{1}\left\|\tilde{\mathrm{Q}}_{s} v\right\|^{2} \frac{d s}{s}\right)^{\frac{1}{2}} \\
& \lesssim\|f\|_{\infty}\|A\|_{\infty}\|u\|\|v\|,
\end{aligned}
$$

where the last inequality follows via our hypothesis and the self-adjointness of $\tilde{D}$. This bounds the first term of (4.4). For the second term, we note that by using duality to compute the norm, we arrive at:

$$
\left|\left\langle\left[\left(\psi_{s} f \psi_{t}\right)(\tilde{\mathrm{D}})-\left(\psi_{s} f \psi_{t}\right)(\mathrm{D})\right] \mathrm{Q}_{t} u, \tilde{\mathrm{Q}}_{s} v\right\rangle\right| \lesssim\|A\|_{\infty}\|f\|_{\infty} \eta(s / t)\left\|\mathrm{Q}_{t} u\right\|\left\|\tilde{\mathrm{Q}}_{s} v\right\|,
$$

where we have used Lemma 4.4. By a similar computation to the previous integral, we obtain that

$$
\int_{0}^{1} \int_{0}^{1}\left|\left\langle\left[\left(\psi_{s} f \psi_{t}\right)(\tilde{\mathrm{D}})-\left(\psi_{s} f \psi_{t}\right)(\mathrm{D})\right] \mathrm{Q}_{t} u, \tilde{\mathrm{Q}}_{s} v\right\rangle\right| \frac{d s}{s} \frac{d t}{t} \lesssim\|A\|_{\infty}\|f\|_{\infty}\|u\|\|v\|
$$


The last term in (4.4) is argued similar to the first term. Combining these estimates together, we obtain that $\|f(\tilde{\mathrm{D}})-f(\mathrm{D})\| \lesssim\|A\|_{\infty}\|f\|_{\infty}$ as claimed.

4.3. Second reduction. In this section, we show that the quadratic estimate

$$
\int_{0}^{1}\left\|\left(\tilde{\mathrm{Q}}_{t}-\mathrm{Q}_{t}\right) u\right\|^{2} \frac{d t}{t} \lesssim\|A\|_{\infty}^{2}\|u\|^{2}
$$

can be reduced to quadratic estimates of the form

$$
\int_{0}^{1}\left\|\mathbf{Q}_{t} S \mathbf{P}_{t} u\right\|^{2} \frac{d t}{t} \lesssim\|A\|_{\infty}^{2}\|u\|^{2}
$$

where the operator $\mathbf{Q}_{t}$ is an operator satisfying quadratic estimates, where $\mathbf{P}_{t}$ is either $\tilde{\mathrm{P}}_{t}$ or $\mathrm{P}_{t}$, and $S$ is an appropriate bounded operator with norm controlled by $\mathrm{C}(\mathcal{M}, \mathcal{V}, \mathrm{D}, \tilde{\mathrm{D}})$. Due to Proposition 4.1, via the decomposition of the difference $\mathrm{D}-\tilde{\mathrm{D}}=A_{1} \nabla+\operatorname{div} A_{2}+A_{3}$, it is clear how the term $\|A\|_{\infty}$ arise in the expression as we note in the following:

$$
\begin{gathered}
\left(\int_{0}^{1}\left\|\left(\tilde{\mathrm{Q}}_{t}-\mathrm{Q}_{t}\right) f\right\|^{2} \frac{d t}{t}\right)^{\frac{1}{2}} \\
\leq\left(\int_{0}^{1}\left\|\tilde{\mathrm{P}}_{t} t A_{1} \nabla \mathrm{P}_{t} f\right\|^{2} \frac{d t}{t}\right)^{\frac{1}{2}}+\left(\int_{0}^{1}\left\|\tilde{\mathrm{P}}_{t} t \operatorname{div} A_{2} \mathrm{P}_{t} f\right\|^{2} \frac{d t}{t}\right)^{\frac{1}{2}} \\
+\left(\int_{0}^{1}\left\|\tilde{\mathrm{P}}_{t} t A_{3} \mathrm{P}_{t} f\right\|^{2} \frac{d t}{t}\right)^{\frac{1}{2}} \\
+\left(\int_{0}^{1}\left\|\tilde{\mathrm{Q}}_{t} t A_{1} \nabla \mathrm{Q}_{t} f\right\|^{2} \frac{d t}{t}\right)^{\frac{1}{2}}+\left(\int_{0}^{1}\left\|\tilde{\mathrm{Q}}_{t} t \operatorname{div} A_{2} \mathrm{Q}_{t} f\right\|^{2} \frac{d t}{t}\right)^{\frac{1}{2}} \\
+\left(\left\|\tilde{\mathrm{Q}}_{t} t A_{3} \mathrm{Q}_{t} f\right\|^{2} \frac{d t}{t}\right)^{\frac{1}{2}} \cdot
\end{gathered}
$$

With this, we obtain the following.

Proposition 4.6. Suppose that

$$
\begin{aligned}
& \int_{0}^{1}\left\|\tilde{\mathrm{Q}}_{t} A_{1} \nabla(\mathrm{iI}+\mathrm{D})^{-1} \mathrm{P}_{t} f\right\|^{2} \frac{d t}{t} \leq C_{1}\|A\|_{\infty}^{2}\|f\|^{2}, \text { and } \\
& \int_{0}^{1}\left\|t \tilde{\mathrm{P}}_{t} \operatorname{div} A_{2} \mathrm{P}_{t} f\right\|^{2} \frac{d t}{t} \leq C_{2}\|A\|_{\infty}^{2}\|f\|^{2}
\end{aligned}
$$

for all $u \in \mathrm{L}^{2}(\mathcal{V})$. Then, for $\omega \in(0, \pi / 2)$ and $\sigma \in(0, \infty)$, whenever $f \in \operatorname{Hol}^{\infty}\left(\mathrm{S}_{\omega, \sigma}^{o}\right)$, we obtain that

$$
\|f(\tilde{\mathrm{D}})-f(\mathrm{D})\| \lesssim\|f\|_{\infty}\|A\|_{\infty}
$$

where the implicit constant depends on $C_{1}, C_{2}$ and $\mathrm{C}(\mathcal{M}, \mathcal{V}, \mathrm{D}, \tilde{\mathrm{D}})$.

Proof. We demonstrate that each term to the right of (4.5) is bounded by

$$
\max \left\{C_{1}, C_{2}\right\}\|A\|_{\infty}^{2}
$$


and apply Proposition 4.5. First note that,

$$
\int_{0}^{1}\left\|\tilde{\mathrm{P}}_{t}\left(t A_{3}\right) \mathrm{P}_{t} f\right\|^{2} \frac{d t}{t} \leq\|A\|_{\infty}^{2} \int_{0}^{1} t^{2}\|f\|^{2} \frac{d t}{t} \leq\|A\|_{\infty}^{2}\|f\|^{2}
$$

and by the same calculation with $\tilde{\mathrm{Q}}_{t}$ and $\mathrm{Q}_{t}$ in place of $\tilde{\mathrm{P}}_{t}$ and $\mathrm{P}_{t}, \int_{0}^{1}\left\|\tilde{\mathrm{Q}}_{t}\left(t A_{3}\right) \mathrm{Q}_{t} f\right\|^{2} \frac{d t}{t} \leq$ $\|A\|_{\infty}^{2}\|f\|^{2}$.

By (2.4) and using the quadratic estimates for $\mathrm{Q}_{t}$,

$$
\begin{aligned}
\int_{0}^{1}\left\|\tilde{\mathrm{P}}_{t}\left(t A_{1} \nabla\right) \mathrm{P}_{t} f\right\|^{2} \frac{d t}{t} & \leq\left\|A_{1}\right\|_{\infty}^{2} \int_{0}^{1}\left\|t \nabla \mathrm{P}_{t} f\right\|^{2} \frac{d t}{t} \\
& \leq 2 \mathrm{C}^{2}\|A\|_{\infty}^{2} \int_{0}^{1}\left(\left\|t \mathrm{DP}_{t} f\right\|^{2}+\left\|t \mathrm{P}_{t} f\right\|^{2}\right) \frac{d t}{t} \\
& \leq 2 \mathrm{C}^{2}\|A\|_{\infty}^{2} \int_{0}^{1}\left(\left\|\mathrm{Q}_{t} f\right\|^{2}+t^{2}\|f\|^{2}\right) \frac{d t}{t} \leq \mathrm{C}^{2}\|A\|_{\infty}^{2}\|f\|^{2}
\end{aligned}
$$

Next, note that for $u \in \mathcal{D}(\operatorname{div})$,

$$
\begin{aligned}
\left\|\tilde{\mathrm{Q}}_{t} t \operatorname{div} u\right\|=\sup _{\|g\|=1}\left\langle\tilde{\mathrm{Q}}_{t} t \operatorname{div} u, g\right\rangle \leq \sup _{\|g\|=1}\|u\|\left\|t \operatorname{div}^{*} \tilde{\mathrm{Q}}_{t} g\right\| \\
\quad \leq \mathrm{C}\|u\| \sup _{\|g\|=1}\left(\left\|t \tilde{\mathrm{D}} \tilde{\mathrm{Q}}_{t} g\right\|+\left\|t \tilde{\mathrm{Q}}_{t} g\right\|\right) \lesssim \mathrm{C}\|u\| .
\end{aligned}
$$

Therefore

$$
\int_{0}^{1}\left\|\tilde{\mathrm{Q}}_{t}\left(t \operatorname{div} A_{2}\right) \mathrm{Q}_{t} f\right\|^{2} \frac{d t}{t} \leq \mathrm{C}^{2}\left\|A_{2}\right\|^{2} \int_{0}^{1}\left\|\mathrm{Q}_{t} f\right\|^{2} \frac{d t}{t} \leq \mathrm{C}^{2}\|A\|_{\infty}^{2}\|f\|^{2} .
$$

The two remaining terms are then handled via the hypothesis. The first term is immediate. For the second term, first we have that

$$
\begin{gathered}
\left(\int_{0}^{1}\left\|\tilde{\mathrm{Q}}_{t}\left(t A_{1} \nabla\right) \mathrm{Q}_{t} f\right\|^{2} \frac{d t}{t}\right)^{\frac{1}{2}}=\left(\int_{0}^{1}\left\|\tilde{\mathrm{Q}}_{t} A_{1} \nabla(\mathrm{iI}+\mathrm{D})^{-1}\left(t(\mathrm{iI}+\mathrm{D}) \mathrm{Q}_{t}\right) f\right\|^{2} \frac{d t}{t}\right)^{\frac{1}{2}} \\
\leq\left(\int_{0}^{1}\left\|\tilde{\mathrm{Q}}_{t} A_{1} \nabla(\mathrm{iI}+\mathrm{D})^{-1} f\right\|^{2} \frac{d t}{t}\right)^{\frac{1}{2}}+\left(\int_{0}^{1}\left\|\tilde{\mathrm{Q}}_{t} A_{1} \nabla(\mathrm{iI}+\mathrm{D})^{-1} \mathrm{P}_{t} f\right\|^{2} \frac{d t}{t}\right)^{\frac{1}{2}} \\
+\left(\int_{0}^{1}\left\|\tilde{\mathrm{Q}}_{t} A_{1} \nabla(\mathrm{iI}+\mathrm{D})^{-1} t \mathrm{Q}_{t} f\right\|^{2} \frac{d t}{t}\right)^{\frac{1}{2}}
\end{gathered}
$$

since $t \mathrm{DQ}_{t}=\mathrm{I}-\mathrm{P}_{t}$. By hypothesis,

$$
\int_{0}^{1}\left\|\tilde{\mathrm{Q}}_{t} A_{1} \nabla(\mathrm{iI}+\mathrm{D})^{-1} \mathrm{P}_{t} f\right\|^{2} \frac{d t}{t} \leq C_{2}\|A\|_{\infty}^{2}\|f\|^{2}
$$

and by the quadratic estimates for $\tilde{\mathrm{Q}}_{t},(2.4)$ and noting that $\left\|\nabla(\mathrm{iI}+\mathrm{D})^{-1} u\right\| \lesssim\|u\|$,

$$
\int_{0}^{1}\left\|\tilde{\mathrm{Q}}_{t} A_{1} \nabla(\mathrm{iI}+\mathrm{D})^{-1} f\right\|^{2} \frac{d t}{t} \leq\left\|A_{1}\right\|_{\infty}^{2}\left\|\nabla(\mathrm{iI}+\mathrm{D})^{-1}\right\|^{2}\|f\|^{2} \lesssim\|A\|_{\infty}^{2}\|f\|^{2} .
$$


For the last term,

$$
\int_{0}^{1}\left\|\tilde{\mathrm{Q}}_{t} A_{1} \nabla(\mathrm{iI}+\mathrm{D})^{-1}\left(t \mathrm{Q}_{t}\right) f\right\|^{2} \frac{d t}{t} \lesssim \int_{0}^{1}\left\|A_{1}\right\|_{\infty}^{2} t^{2}\|f\|^{2} \frac{d t}{t} \leq\|A\|_{\infty}^{2}\|f\|^{2} .
$$

This finishes the proof.

We conclude this section by remarking that in typical applications, as we will see in $\S 5$, the constants $C_{1}$ and $C_{2}$ themselves will depend on $\mathrm{C}(\mathcal{M}, \mathcal{V}, \mathrm{D}, \tilde{\mathrm{D}})$.

\section{Quadratic estimates}

In this section, we prove the quadratic estimates in the hypothesis of Proposition 4.6. We consider both quadratic estimates appearing as the hypothesis of this proposition combined into the general form

$$
\int_{0}^{1}\left\|\mathrm{Q}_{t} S \mathrm{P}_{t} f\right\|^{2} \frac{d t}{t} \lesssim\|A\|_{\infty}^{2}\|f\|^{2},
$$

where $S: \mathrm{L}^{2}(\mathcal{V}) \rightarrow \mathrm{L}^{2}(\mathcal{W})$ and $\mathrm{Q}_{t}: \mathrm{L}^{2}(\mathcal{W}) \rightarrow \mathrm{L}^{2}(\mathcal{V})$, where $\mathcal{W}$ is an auxiliary vector bundle and $\mathbf{Q}_{t}$ is a family of operators with sufficient decay.

It is well known in harmonic analysis, going back to the counter example in [21] by the second author to the abstract Kato square root conjecture, that estimates of the form (5.1), even for multipliers $S$, cannot be proved only using operator theory methods such as those in $\S 4$. Instead one needs to apply harmonic analysis to exploit the differential structure of the operators and the space. It is here that we require the full list (A1)-(A9) of assumptions.

The purpose of considering an abstract estimate of this form is due to the fact that to satisfy the hypothesis of Proposition 4.6, we are required to prove two different quadratic estimates with the choice of operators $S=\mathrm{I}$ for $\mathrm{Q}_{t}=\tilde{\mathrm{P}}_{t} \operatorname{div} A_{2}$ and $S=\nabla(\mathrm{iI}+\mathrm{D})^{-1}$ for $\mathrm{Q}_{t}=\tilde{\mathrm{Q}}_{t} A_{1}$. Therefore, in order to make the presentation clearer for the reader, we combine these two estimates into a single estimate. Note that while it may seem that the first choice for $\mathrm{Q}_{t}$ and $S$ is an easy estimate, the fact that the operator $\mathrm{P}_{t}$ appears in the required quadratic estimate to the right of $\mathbf{Q}_{t}$ precisely means that this estimate that cannot be handled by operator theory methods alone.

In what will follow, the key is to reduce the estimate (5.1) to a Carleson measure estimate. We will impose further restrictions on $S$ as required in the analysis that will follow.

5.1. Dyadic grids and GBG frames. A central consequence of the growth assumption $\left(\mathrm{E}_{\mathrm{loc}}\right)$ is that it affords us with a dyadic decomposition. This is illustrated in the following theorem.

Theorem 5.1 (Existence of a truncated dyadic structure). Suppose that $(\mathcal{M}, \mathrm{g})$ satisfies $\left(\mathrm{E}_{\mathrm{loc}}\right)$. Then, there exist countably many index sets $I_{k}$, a countable collection 
of open subsets $\left\{Q_{\alpha}^{k} \subset \mathcal{M}: \alpha \in I_{k}, k \in \mathbb{N}\right\}$, points $z_{\alpha}^{k} \in Q_{\alpha}^{k}$ (called the centre of $\left.Q_{\alpha}^{k}\right)$, and constants $\delta \in(0,1), a_{0}>0, \eta>0$ and $C_{1}, C_{2}<\infty$ satisfying:

(i) for all $k \in \mathbb{N}, \mu\left(\mathcal{M} \backslash \cup_{\alpha} Q_{\alpha}^{k}\right)=0$,

(ii) if $l \geq k$, then either $Q_{\beta}^{l} \subset Q_{\alpha}^{k}$ or $Q_{\beta}^{l} \cap Q_{\alpha}^{k}=\varnothing$,

(iii) for each $(k, \alpha)$ and each $l<k$ there exists a unique $\beta$ such that $Q_{\alpha}^{k} \subset Q_{\beta}^{l}$,

(iv) $\operatorname{diam} Q_{\alpha}^{k}<C_{1} \delta^{k}$,

(v) $\mathrm{B}\left(z_{\alpha}^{k}, a_{0} \delta^{k}\right) \subset Q_{\alpha}^{k}$,

(vi) for all $k, \alpha$ and for all $t>0, \mu\left\{x \in Q_{\alpha}^{k}: d\left(x, \mathcal{M} \backslash Q_{\alpha}^{k}\right) \leq t \delta^{k}\right\} \leq C_{2} t^{\eta} \mu\left(Q_{\alpha}^{k}\right)$.

This theorem was first proved by Christ in [12] for $k \in \mathbb{Z}$ (i.e. untruncated) for doubling measure metric spaces. It was generalised by Morris in [23] to our particular setting.

In what is to follow we couple this dyadic grid with the notion of GBG for the vector bundle $(\mathcal{V}, \mathrm{h})$. We encourage the reader to assume familiarity with the constants $C_{1}, a_{0}$ and $\delta$ from Theorem 5.1. We remark that terminology we define below first arose in the harmonic analysis of the Kato square root problem on vector bundles in $[6]$.

We define and note the following:

- fix $\mathrm{J} \in \mathbb{N}$ such that $C_{1} \delta^{\mathrm{J}} \leq \rho / 5$ where $\rho$ is from Definition (2.3),

- let $\mathrm{t}_{\mathrm{S}}=\delta^{J}$ which we call the scale,

- whenever $j \geq \mathrm{J}, \mathscr{Q}^{j}$ denotes the set of cubes $Q_{\alpha}^{j}$,

- define $\mathscr{Q}=\cup_{j \geq \mathrm{J}} \mathscr{Q}^{j}$,

- whenever $t \leq \mathrm{t}_{\mathrm{S}}$, we define $\mathscr{Q}_{t}=\mathscr{Q}^{j}$ if $\delta^{j+1}<t \leq \delta^{j}$,

- the length of a cube $Q \in \mathscr{Q}^{j}$ is $\ell(Q)=\delta^{j}$,

- for any $Q \in \mathscr{Q}^{j}$, there exists a unique ancestor cube $\widehat{Q} \in \mathscr{Q}^{\mathrm{J}}$ such that $Q \subset \widehat{Q}$, and the cube $\widehat{Q}$ is called the $G B G$ cube of $Q$.

The following notion allows us to couple the dyadic structure with the GBG condition yielding "good" coordinates for $\mathcal{V}$ that enable us to import tools from Euclidean harmonic analysis to the vector bundle setting. In the following definition, for a cube $Q=Q_{\alpha}^{j} \in \mathscr{Q}^{j}$, we define $x_{Q}=z_{\alpha}^{j}$ and call this the centre of the cube.

Definition 5.2. We call the following system of $G B G$ trivialisations

$$
\mathscr{C}=\left\{\psi: \mathrm{B}\left(x_{Q}, \rho\right) \times \mathbb{C}^{N} \rightarrow \pi_{\mathcal{V}}^{-1}\left(\mathrm{~B}\left(x_{Q}, \rho\right)\right), Q \in \mathscr{Q}^{J}\right\}
$$

the GBG coordinates. Moreover, we let

$$
\mathscr{C}_{\mathrm{J}}=\left\{\left.\psi\right|_{Q}: Q \times \mathbb{C}^{N} \rightarrow \pi_{\mathcal{V}}^{-1}(Q), \psi \in \mathscr{C}\right\}
$$

which we call the dyadic GBG coordinates. For an arbitrary cube $Q \in \mathscr{Q}$, the $G B G$ coordinates of $Q$ are the $G B G$ coordinates of the $G B G$ cube $\widehat{Q}$. 
An important tool in harmonic analysis is to be able to perform averages, which requires a notion of integration. In a general vector bundle, this is not a well-defined notion under transformations. However, by using the GBG structure, we define the notion of cube integration, as a map $\mathrm{B}\left(x_{\widehat{Q}}, \rho\right) \times \mathscr{Q} \ni(x, Q) \mapsto\left(\int_{Q} \cdot\right)(x)$. For $u \in \mathrm{L}_{\text {loc }}^{1}(\mathcal{V})$, and $y \in \mathrm{B}\left(x_{\widehat{Q}}, \rho\right)$ we write

$$
\left(\int_{Q} u d \mu\right)(y)=\left(\int_{Q} u_{i} d \mu\right) e^{i}(y)
$$

where $u=u_{i} e^{i}$ in the GBG coordinates of $Q$. Note that this integral is only defined in $\mathrm{B}\left(x_{\widehat{Q}}, \rho\right)$. We then define the cube average $u_{Q} \in \mathrm{L}^{\infty}(\mathcal{V})$ of some $u \in \mathrm{L}_{\text {loc }}^{1}(\mathcal{V})$ as as

$$
u_{Q}(y)= \begin{cases}f_{Q} u d \mu & y \in \mathrm{B}\left(x_{\widehat{Q}}, \rho\right) \\ 0 & y \notin \mathrm{B}\left(x_{\widehat{Q}}, \rho\right) .\end{cases}
$$

Lastly, for each $t>0$, we define the dyadic averaging operator $\mathbb{E}_{t}: \mathrm{L}_{\text {loc }}^{1}(\mathcal{V}) \rightarrow \mathrm{L}_{\text {loc }}^{1}(\mathcal{V})$ by

$$
\mathbb{E}_{t} u(x)=\left(f_{Q} u d \mu\right)(x)
$$

where $Q \in \mathscr{Q}_{t}$ and $x \in Q$. This defines $\mathbb{E}_{t} u(x)$ for $x$-a.e. on $\mathcal{M}$. We remark that this operator is well defined, and that $\mathbb{E}_{t} u(x)$ on each $Q \in \mathscr{Q}_{t}$. Moreover, $\mathbb{E}_{t}: \mathrm{L}^{2}(\mathcal{V}) \rightarrow \mathrm{L}^{2}(\mathcal{V})$ is bounded uniformly for $t \leq \mathrm{t}_{\mathrm{S}}$ with the bound depending on the constant $C$ arising in the GBG criterion.

5.2. Harmonic analysis. Let us assume that $\mathcal{V}$ and $\mathcal{W}$ are two vector bundles both satisfying the GBG condition and on taking a minimum of the GBG radius of the two bundles, assume that $\mathcal{V}$ and $\mathcal{W}$ share the same GBG radius. Let $\mathrm{Q}_{t}$ : $\mathrm{L}^{2}(\mathcal{W}) \rightarrow \mathrm{L}^{2}(\mathcal{V})$ be a family of operators uniformly bounded in $t \in(0,1]$. The $\mathbf{Q}_{t}$ we consider will naturally contain the coefficients $A_{i}$ as a factor.

On defining $\langle a\rangle=\max \{1, a\}$, we assume that $\mathbf{Q}_{t}$ satisfies off-diagonal estimates: there exists $C_{\mathbf{Q}}>0$ such that, for each $M>0$, there exists a constant $C_{\Delta, M}>0$ satisfying:

$$
\begin{aligned}
\left\|\chi_{E} \mathbf{Q}_{t}\left(\chi_{F} u\right)\right\|_{L^{2}(\mathcal{V})} \leq C_{\Delta, M}\|A\|_{\infty}^{2}\left\langle\frac{\rho(E, F)}{t}\right\rangle^{-M} & \\
& \quad \exp \left(-C_{\mathbf{Q}} \frac{\rho(E, F)}{t}\right)\left\|\chi_{F} u\right\|_{\mathrm{L}^{2}(\mathcal{W})}
\end{aligned}
$$

for every Borel set $E, F \subset \mathcal{M}$ and $u \in \mathrm{L}^{2}(\mathcal{W})$. Moreover, we assume that $\mathrm{Q}_{t}$ satisfies quadratic estimates, by which we mean there exists $C_{\mathbf{Q}}^{\prime}>0$ so that

$$
\int_{0}^{1}\left\|\mathbf{Q}_{t} f\right\|^{2} \frac{d t}{t} \leq C_{\mathbf{Q}}^{\prime}\|A\|_{\infty}^{2}\|f\|^{2}
$$

for all $f \in \mathrm{L}^{2}(\mathcal{V})$. 
Recalling the constants $c_{E}$ and $\kappa$ appearing in $\left(\mathrm{E}_{\mathrm{loc}}\right)$, Lemma 4.4 in [23] states that, whenever $M>\kappa$ and $m>c_{E} / t$, we have

$$
\sup _{Q^{\prime} \in \mathscr{Q}_{t}} \sum_{Q \in \mathscr{Q}_{t}} \frac{\mu(Q)}{\mu\left(Q^{\prime}\right)}\left\langle\frac{\rho\left(Q, Q^{\prime}\right)}{t}\right\rangle^{-M} \exp \left(-m \frac{\rho\left(Q, Q^{\prime}\right)}{t}\right) \lesssim 1 .
$$

As a consequence, arguing exactly as in Lemma 5.3 in [23], we obtain that $\mathbf{Q}_{t}$ extends to a bounded operator $\mathbf{Q}_{t}: \mathrm{L}^{\infty}(\mathcal{W}) \rightarrow \mathrm{L}_{\text {loc }}^{2}(\mathcal{V})$ with $c>0$ such that

$$
\left\|\mathbf{Q}_{t} u\right\|_{\mathrm{L}^{2}(Q ; \mathcal{V})}^{2} \leq c\|A\|_{\infty}^{2} \mu(Q)\|u\|_{\mathrm{L}^{\infty}(\mathcal{W})}^{2}
$$

whenever $t \in\left(0, \mathrm{t}_{\mathrm{H}}(\mathbf{Q})\right]$, where

$$
\mathrm{t}_{\mathrm{H}}(\mathbf{Q})=\min \left\{\mathrm{t}_{\mathrm{S}},\left\langle 2\left\langle\delta / C_{1}\right\rangle^{-1} c_{E} / C_{\mathbf{Q}}\right\rangle^{-1}\right\}
$$

we call the harmonic analysis scale of $\mathbf{Q}_{t}$.

In the harmonic analysis, constant functions are often required to extract principal parts of operators. Under the guise of the GBG coordinate system, we are able to define a notion of a constant section, locally, of $\mathcal{V}$. Let $x \in Q \in \mathscr{Q}$ and $w \in \mathcal{V}_{x} \cong \mathbb{C}^{N}$, and write $w=w_{i} e^{i}(x)$ in the GBG frame $\left\{e^{i}(x)\right\}$ associated to $Q$. We then define the constant extension of $w$ by

$$
w^{c}(y)= \begin{cases}w_{i} e^{i}(y) & y \in \mathrm{B}\left(x_{\widehat{Q}}, \rho\right) \\ 0 & y \notin \mathrm{B}\left(x_{\widehat{Q}}, \rho\right),\end{cases}
$$

and we note that $w^{c} \in \mathrm{L}^{\infty}(\mathcal{V})$.

For $x \in Q \in \mathscr{Q}$, and $w \in \mathcal{V}_{x}$, with GBG constant extension $w^{c} \in \mathrm{L}^{\infty}(\mathcal{V})$, we define the principal part of $\mathbf{Q}_{t}$ by

$$
\gamma_{t}^{\mathbf{Q}}(x) w=\left(\mathbf{Q}_{t} w^{c}\right)(x)
$$

It is easy to see that the principal part is a well defined operator $\gamma_{t}^{\mathrm{Q}}(x): \mathcal{W}_{x} \rightarrow \mathcal{V}_{x}$ for almost-every $x \in \mathcal{M}$. For convenience, we often write $\gamma_{t}$ instead of $\gamma_{t}^{\mathbf{Q}}$.

We note that as a consequence of (5.7) that

$$
f_{Q}\left|\gamma_{t}(x)\right|^{2} d \mu(x) \leq\|A\|_{\infty}^{2} \quad \text { and } \quad \sup _{t \in\left(0, t_{\mathrm{H}}(\mathbf{Q})\right]}\left\|\gamma_{t} \mathbb{E}_{t}\right\| \lesssim\|A\|_{\infty} .
$$

for all $t \in\left(0, \mathrm{t}_{\mathrm{H}}(\mathbf{Q})\right]$. This can be seen by a similar argument to that found in [23] or $[6]$.

With this notation in hand, we split the quadratic from (5.1) as follows:

$$
\begin{aligned}
\int_{0}^{1}\left\|\mathrm{Q}_{t} S \mathrm{P}_{t} f\right\|^{2} \frac{d t}{t} \lesssim \int_{0}^{1} & \left\|\left(\mathrm{Q}_{t}-\gamma_{t} \mathbb{E}_{t}\right) S \mathrm{P}_{t} f\right\|^{2} \frac{d t}{t} \\
& +\int_{0}^{1}\left\|\gamma_{t} \mathbb{E}_{t} S\left(\mathrm{I}-\mathrm{P}_{t}\right) f\right\|^{2} \frac{d t}{t}+\int_{0}^{1}\left\|\gamma_{t} \mathbb{E}_{t} S f\right\|^{2} \frac{d t}{t}
\end{aligned}
$$

We call the first term on the left of (5.11) the principal part, the second term the cancellation part and the last term the Carleson part. 
From here on, we let the standing assumptions throughout the remainder of this section be (A1)-(A9).

5.3. The principal part term. In this subsection, under some additional conditions on $S$, we bound the principal part. The first thing we observe and require is a Poincaré inequality that is bootstrapped from the Poincaré inequality for functions.

Lemma 5.3 (Dyadic Poincaré Lemma). There exists $C_{P}>0$ such that

$$
\int_{\mathrm{B}}\left|u-u_{Q}\right|^{2} d \mu \leq C_{P} r^{\kappa} \mathrm{e}^{c_{E} r t}(r t)^{2} \int_{\mathrm{B}}\left(|\nabla u|^{2}+|u|^{2}\right) d \mu
$$

for $u \in \mathrm{W}^{1,2}(\mathcal{V})$, for all balls $\mathrm{B}=\mathrm{B}\left(x_{Q}, r t\right)$ with $r \geq C_{1} / \delta$ (with the constant $C_{1}$ and $\delta$ from Theorem 5.1) where $Q \in \mathscr{Q}_{t}$ with $t \leq \mathrm{t}_{\mathrm{S}}$ (with $\mathscr{Q}_{t}$ and $\mathrm{t}_{\mathrm{S}}$ from (5.2)). The constant $C_{P}$ depends on $\mathrm{C}(\mathcal{M}, \mathcal{V}, \mathrm{D}, \tilde{\mathrm{D}})$.

The proof of this lemma proceeds similar to the proof of Proposition 5.3 in [6].

Proposition 5.4 (Principal part). Let $\left(\mathcal{W}, \mathrm{h}_{\mathcal{W}}, \nabla^{\mathcal{W}}\right)$ be another vector bundle satisfying $\mathrm{C}^{0,1}-G B G$ and suppose there exists $C_{G, \mathcal{W}}$ such that in each $G B G$ frame $\left\{e^{i}\right\}$ for $\mathcal{W},\left|\nabla^{\mathcal{W}} e^{i}(x)\right| \leq C_{G, \mathcal{W}}$ for almost-every $x$. Let $\mathrm{Q}_{t}: \mathrm{L}^{2}(\mathcal{W}) \rightarrow \mathrm{L}^{2}(\mathcal{V})$ be a family of operators uniformly bounded in $t \in(0,1]$ satisfying $(5.4)$ and $(5.5)$, and suppose $S: \mathrm{L}^{2}(\mathcal{V}) \rightarrow \mathrm{L}^{2}(\mathcal{W})$ is a bounded operator for which

$$
\left\|\nabla^{\mathcal{W}} S v\right\| \leq C_{S}\|v\|_{\mathrm{W}^{1,2}}
$$

for some $C_{S}>0$ and $v \in \mathrm{W}^{1,2}(\mathcal{V})$. Then, whenever $u \in \mathrm{L}^{2}(\mathcal{V})$,

$$
\int_{0}^{t_{1}(\mathbf{Q})}\left\|\left(\mathbf{Q}_{t}-\gamma_{t} \mathbb{E}_{t}\right) S \mathrm{P}_{t} u\right\|^{2} \frac{d t}{t} \lesssim\|A\|_{\infty}^{2}\|u\|^{2},
$$

where $_{1}(\mathbf{Q})=\min \left\{\mathrm{t}_{\mathrm{H}}(\mathbf{Q}), C_{\mathbf{Q}} /\left(11 c_{E}\right)\right\}$. The implicit constant depends on $C_{G, W}, C_{S}$, $C_{\Delta, \kappa+3}$ from (5.4), $C_{\mathbf{Q}}^{\prime}$ from (5.5) and $\mathrm{C}(\mathcal{M}, \mathcal{V}, \mathrm{D}, \tilde{\mathrm{D}})$.

Remark 5.5. We allow for an auxiliary vector bundle $\mathcal{W}$ in this proposition since, in the proof of Theorem 2.4, we are required to invoke this with different choices for $\mathcal{W}$. We will see later that the constants $C_{S}, C_{G, \mathcal{W}}, C_{\Delta, \kappa+3}$ and $C_{\mathbf{Q}}^{\prime}$ are themselves dependent on $\mathrm{C}(\mathcal{M}, \mathcal{V}, \mathrm{D}, \tilde{\mathrm{D}})$.

Proof. The proof proceeds similar to Proposition 8.4 in [6], by replacing their $\mathbf{Q}_{t}^{B}$ with our $\mathbf{Q}_{t}$.

Set $v=S \mathrm{P}_{t} u$. First, note from (5.3) that $\mathbb{E}_{t} v(x)=v_{Q}(x)$ for $x \in Q$, and so

$$
\left\|\left(\mathbf{Q}_{t}-\gamma_{t} \mathbb{E}_{t}\right) v\right\|^{2}=\sum_{Q \in \mathscr{Q}_{t}}\left\|\mathbf{Q}_{t}\left(v-v_{Q}\right)\right\|_{\mathrm{L}^{2}(Q)}^{2} .
$$


Letting $\mathrm{B}_{Q}=\mathrm{B}\left(x_{Q}, C_{1} / \delta t\right), C_{j}(Q)=2^{j+1} \mathrm{~B}_{Q} \backslash 2^{j} \mathrm{~B}_{Q}$, and on invoking (5.4) for $\mathbf{Q}_{t}$ and for some $M>0$ to be chosen later, we obtain that

$$
\begin{aligned}
& \int_{Q}\left|\mathbf{Q}_{t}\left(v-v_{Q}\right)\right|^{2} d \mu \\
& \quad \lesssim\|A\|_{\infty}^{2}\left(\sum_{j=0}^{\infty}\left\langle\frac{\rho\left(Q, C_{j}(Q)\right)}{t}\right\rangle^{-M} \exp \left(-C_{\mathbf{Q}} \frac{\rho\left(Q, C_{j}(Q)\right)}{t}\right)\left\|v-v_{Q}\right\|_{L^{2}\left(C_{j}(Q)\right)}\right)^{2} .
\end{aligned}
$$

By (4.1) in [23], we have

$$
2^{j} \frac{C_{1}}{\delta} t \leq \rho\left(x_{Q}, C_{j}(Q)\right) \leq \rho\left(Q, C_{j}(Q)\right)+\operatorname{diam} Q
$$

and therefore

$$
\begin{aligned}
& \left\langle\frac{\rho\left(Q, C_{j}(Q)\right)}{t}\right\rangle^{-M} \lesssim 2^{-M(j+1)} \quad \text { and } \\
& \exp \left(-C_{\mathbf{Q}} \frac{\rho\left(Q, C_{j}(Q)\right)}{t}\right) \lesssim \exp \left(-\frac{C_{\mathbf{Q}} C_{1}}{4 \delta} 2^{j+1}\right)
\end{aligned}
$$

for all $j \geq 0$. Thus, by Cauchy-Schwartz inequality applied to (5.12), we obtain that

$$
\begin{aligned}
& \int_{Q}\left|\mathbf{Q}_{t}\left(v-v_{Q}\right)\right|^{2} d \mu \\
& \quad \lesssim\|A\|_{\infty}^{2} \sum_{j=0}^{\infty} 2^{-M(j+1)} \exp \left(-C_{\mathbf{Q}} \frac{C_{1}}{2 \delta} 2^{j+1}\right) \int_{C_{j}(Q)}\left|v-v_{Q}\right|^{2} d \mu .
\end{aligned}
$$

On observing that $C_{j}(Q) \subset 2^{j+1} \mathrm{~B}_{Q}, v \in \mathrm{W}^{1,2}(\mathcal{W}), S: \mathrm{W}^{1,2}(\mathcal{V}) \rightarrow \mathrm{W}^{1,2}(\mathcal{W})$, and since $\left(\mathcal{W}, \mathrm{h}_{\mathcal{W}}, \nabla^{\mathcal{W}}\right)$ has $\mathrm{C}^{0,1}$-GBG with $\left|\nabla^{\mathcal{W}} e^{i}\right| \leq C_{G}$ almost-everywhere, we apply Lemma 5.3 to obtain

$$
\begin{aligned}
\int_{C_{j}(Q)} & \left|v-v_{Q}\right|^{2} d \mu \\
& \lesssim\left(\frac{C_{1}}{\delta}\right)^{\kappa+2} \exp \left(\frac{c_{E} C_{1}}{\delta} 2^{j+1} t\right) 2^{2(j+1)} t^{2} \int_{2^{j+1} \mathrm{~B}_{Q}}\left(\left|\nabla^{\mathcal{W}} v\right|^{2}+|v|^{2}\right) d \mu
\end{aligned}
$$

To estimate the term

$$
\int_{2^{j+1} \mathrm{~B}_{Q}}\left(\left|\nabla^{\mathcal{W}} v\right|^{2}+|v|^{2}\right) d \mu=\int \chi_{2^{j+1} \mathrm{~B}_{Q}}\left(\left|\nabla^{\mathcal{W}} v\right|^{2}+|v|^{2}\right) d \mu
$$

we use Lemma 8.3 in [6], which states that whenever $r>0$ and $\left\{\mathrm{B}_{j}=\mathrm{B}\left(x_{j}, r\right)\right\}$ is a disjoint collection of balls, then for every $\eta \geq 1$,

$$
\sum_{j} \chi_{\eta \mathrm{B}_{j}} \lesssim \eta^{\kappa} \mathrm{e}^{4 c_{E} \eta \kappa}
$$


where the implicit constant depends on ( $\left.\mathrm{E}_{\mathrm{loc}}\right)$. We apply this on setting $r=a_{0} t$ and $\eta=2^{j+1} C_{1} /\left(\delta a_{0}\right)$ so that $\left\{\mathrm{B}\left(x_{Q}, a_{0} t\right)\right\}$ is disjoint to obtain the bound

$$
\chi_{2^{j+1} \mathrm{~B}_{Q}} \lesssim 2^{\kappa(j+1)} \exp \left(\frac{4 c_{E} C_{1}}{\delta} 2^{j+1} t\right) .
$$

On combining estimates (5.13), (5.15) and (5.16) with (5.14),

$$
\begin{gathered}
\sum_{Q \in \mathscr{Q}_{t}} \int_{Q}\left|\mathbf{Q}_{t}\left(v-v_{Q}\right)\right|^{2} d \mu \\
\lesssim\|A\|_{\infty}^{2} \sum_{j=0}^{\infty} 2^{-(M-\kappa-2)(j+1)} \exp \left(-\frac{C_{1}}{2 \delta}\left(C_{\mathbf{Q}}-10 c_{E} t\right) 2^{j+1}\right) \\
t^{2}\left(\left\|\nabla^{\mathcal{W}} v\right\|^{2}+\|v\|^{2}\right) .
\end{gathered}
$$

This sum converges by choosing $M>\kappa+2$ and for $t \leq \frac{C_{\mathbf{Q}}}{11 c_{E}}$. Then, on setting $t_{1}(\mathbf{Q})=\min \left\{\mathrm{t}_{\mathrm{H}}(\mathbf{Q}), C_{\mathbf{Q}} /\left(11 c_{E}\right)\right\}$, and recalling that $v=S \mathrm{P}_{t} u$,

$$
\begin{aligned}
\int_{0}^{t_{1}(\mathbf{Q})} & \left\|\left(\mathbf{Q}_{t}-\gamma_{t} \mathbb{E}_{t}\right) S \mathrm{P}_{t} u\right\|^{2} \frac{d t}{t} \\
& \lesssim\|A\|_{\infty}^{2} \int_{0}^{t_{1}(\mathbf{Q})} t^{2}\left\|\nabla^{\mathcal{W}} S \mathrm{P}_{t} u\right\|^{2} \frac{d t}{t}+\|A\|_{\infty}^{2} \int_{0}^{t_{1}(\mathbf{Q})} t^{2}\left\|S \mathrm{P}_{t} u\right\|^{2} \frac{d t}{t} \\
& \lesssim\|A\|_{\infty}^{2} \int_{0}^{t_{1}(\mathbf{Q})}\left(t^{2}\left\|\nabla^{\mathcal{V}} \mathrm{P}_{t} u\right\|^{2}+\left\|\mathrm{P}_{t} u\right\|^{2}\right) \frac{d t}{t}+\|A\|_{\infty}^{2} \int_{0}^{t_{1}(\mathbf{Q})} t^{2}\left\|S \mathrm{P}_{t} u\right\|^{2} \frac{d t}{t} \\
& \lesssim\|A\|_{\infty}^{2}\|u\|^{2}+\|A\|_{\infty}^{2} \int_{0}^{t_{1}(\mathbf{Q})} t^{2}\left\|\mathrm{DP}_{t} u\right\|^{2} \frac{d t}{t} \\
& \lesssim\|A\|_{\infty}^{2}\|u\|^{2},
\end{aligned}
$$

where the second inequality follows from the assumption $\left\|\nabla^{\mathcal{W}} S w\right\|^{2} \lesssim\left\|\nabla^{\mathcal{V}} w\right\|^{2}+$ $\|w\|^{2}$, the third inequality from the boundedness of $S: \mathrm{L}^{2}(\mathcal{V}) \rightarrow \mathrm{L}^{2}(\mathcal{W})$ and $(2.4)$, and the last inequality from the fact that $t \mathrm{DP}_{t}=\mathrm{Q}_{t}$ satisfies quadratic estimates.

5.4. The cancellation term. In this subsection, we estimate the cancellation term. First, we observe the following.

Lemma 5.6. On each dyadic cube $Q$, and for each $u \in \mathrm{W}^{1,2}(\mathcal{V})$ with spt $u \subset Q$, we have that

$$
\left|\int_{Q} \mathrm{D} u d \mu\right| \lesssim \mu(Q)^{\frac{1}{2}}\|u\| .
$$

The implicit constant depends on $\mathrm{C}(\mathcal{M}, \mathcal{V}, \mathrm{D}, \tilde{\mathrm{D}})$.

Proof. Let $u=u_{i} e^{i}$ inside the GBG frame associated to $Q$, and let $\left\{v_{j}\right\}$ be the GBG frame for $\mathrm{T} \mathcal{M}$. Then, from $(2.2)$, we write in this frame

$$
\mathrm{D} u=\left(\alpha_{l}^{j k} \nabla_{v_{j}} u_{k}+u_{i} \omega_{l}^{i}\right) e^{l},
$$


and for a bounded Lipschitz $\eta: \mathcal{M} \rightarrow \mathbb{R}$,

$$
\begin{aligned}
{[\eta, \mathrm{D}] u=} & \eta \mathrm{D} u-\mathrm{D}(\eta u) \\
& \left.=\eta\left(\alpha_{l}^{j k} \nabla_{v_{j}} u_{k}+u_{i} \omega_{l}^{i}\right) e^{l}-\alpha_{l}^{j k} \nabla_{v_{j}}\left(\eta u_{k}\right)+\eta u_{i} \omega_{l}^{i}\right) e^{l}=\alpha_{l}^{j k}\left(\nabla_{v_{j}} \eta\right) u_{k} e^{l},
\end{aligned}
$$

almost-everywhere inside the GBG frame. By choosing $\eta$ appropriately, i.e., $\nabla \eta=$ $v_{j}$

$$
\sum_{j, k, l}\left|\alpha_{l}^{j k}\right|^{2} \lesssim \operatorname{dim}(\mathcal{V})
$$

Moreover, from (A7), we deduce the bound

$$
\sum_{k}\left|\omega_{k}^{i}\right|^{2} \simeq\left|\omega_{k}^{i} e^{k}\right|^{2}=\left|\mathrm{D} e^{i}\right|^{2} \leq c_{\mathrm{D}, \mathcal{V}}
$$

Before we proceed, we note that the assumption $\left|\nabla e_{i}\right| \leq C_{G, \mathcal{V}}$ implies that $\left|\nabla_{\nu_{j}} \mathrm{~h}_{i j}\right| \lesssim$ 1 almost-everywhere since we assume that $\mathrm{h}$ and $\nabla$ are compatible almost-everywhere. The implicit constant here depends only on of $C_{G, \mathcal{V}}$ and $C_{\mathcal{V}}$.

Now, let $\mathrm{h}^{*}=\mathrm{h}_{i j} e^{i} \otimes e^{j}$ denote the induced metric for $\mathcal{V}^{*}$ from $\mathrm{h}=\mathrm{h}^{i j} e_{i} \otimes e_{j}$, where $e^{i}\left(e_{j}\right)=\delta_{i j}$. Now, note that we can write a section $f \in \mathrm{L}_{\text {loc }}^{1}(\mathcal{V})$ in $\left\{e^{i}\right\}$ as $f=f_{i} e^{i}=\mathrm{h}\left(f, \mathrm{~h}_{i k} e^{i}\right) e^{k}$, and on choosing $\psi$ to be a Lipschitz function supported inside the trivialisation for the frame $\left\{e_{i}\right\}$, with $\psi \equiv 1$ on $Q$ we compute using the fact that $u=0$ on spt $\nabla \psi$

$$
\begin{aligned}
& \int_{Q} \mathrm{D} u=\int_{Q} \mathrm{~h}(\mathrm{D} u\left., \psi \mathrm{h}_{i k} e^{i}\right) e^{k}=\int_{\mathcal{M}} \mathrm{h}\left(\mathrm{D} u, \psi \mathrm{h}_{i k} e^{i}\right) e^{k}=\int_{\mathcal{M}} \mathrm{h}\left(u, \mathrm{D}\left(\psi \mathrm{h}_{i k} e^{i}\right)\right) e^{k} \\
&=\int_{Q} \mathrm{~h}\left(u, \mathrm{D}\left(\mathrm{h}_{i k} e^{i}\right)\right) e^{k}=\int_{Q} \mathrm{~h}\left(u,\left(\alpha_{l}^{j m} \nabla_{v_{j}} \mathrm{~h}_{m k}+\mathrm{h}_{i k} \omega_{l}^{i}\right) e^{l}\right) e^{k} .
\end{aligned}
$$

Therefore,

$$
\begin{aligned}
\left|\int_{Q} \mathrm{D} u\right| \lesssim \int_{Q}|u| & \left.\sum_{k, m, l}\left|\alpha_{l}^{j m} \nabla_{v_{j}} \mathrm{~h}_{m k}\right|+\int_{Q}|u| \sum_{k, m} \mid\left(\mathrm{h}_{i k} \omega_{m}^{i}\right) e^{m}\right) \mid \\
& \lesssim \int_{Q}|u|=\int_{\mathcal{M}} \chi_{Q}|u| \leq\left(\int_{\mathcal{M}} \chi_{Q}^{2}\right)^{\frac{1}{2}}\left(\int_{\mathcal{M}}|u|^{2}\right)^{\frac{1}{2}}=\mu(Q)^{\frac{1}{2}}\|u\|,
\end{aligned}
$$

using the proved bounds on $\alpha_{l}^{j k}$ and $\omega_{j}^{i}$ and bounds on $\nabla_{v_{j}} \mathrm{~h}_{k l}$ and $\mathrm{h}_{k l}$ from (A5).

Lemma 5.7. On each dyadic cube $Q$, each $u \in \mathrm{W}^{1,2}(\mathcal{V})$ and $v \in \mathcal{D}(\operatorname{div})$ with spt $v$, spt $u \subset Q$, we have that

$$
\left|\int_{Q} \nabla u d \mu\right| \lesssim \mu(Q)^{\frac{1}{2}}\|u\| \quad \text { and } \quad\left|\int_{Q} \operatorname{div} v d \mu\right| \lesssim \mu(Q)^{\frac{1}{2}}\|v\| .
$$

The implicit constants depend on $\mathrm{C}(\mathcal{M}, \mathcal{V}, \mathrm{D}, \tilde{\mathrm{D}})$.

This lemma is proved very similar to Lemma 5.6. For a comprehensive outline of the proof, we consult the reader to the proof of Theorem 6.2 in [6]. Although the metrics in [6] are assumed to be smooth, it is easy to verify that our assumption of $\mathrm{C}^{0,1}$ regularity of the metric suffices in their proof. 
The following is a generalisation of a key estimate in [3].

Lemma 5.8 (Cancellation lemma). Let $\Upsilon$ be either one of $\mathrm{D}, \tilde{\mathrm{D}}, \nabla$, or div. Then,

$$
\left|f_{Q} \Upsilon u d \mu\right|^{2} \lesssim \frac{1}{\ell(Q)^{\eta}}\left(f_{Q}|u|^{2} d \mu\right)^{\frac{\eta}{2}}\left(f_{Q}|\Upsilon u|^{2}\right)^{1-\frac{\eta}{2}}+f_{Q}|u|^{2},
$$

for all $u \in \mathcal{D}(\Upsilon), Q \in \mathscr{Q}, t \in\left(0, \mathrm{t}_{\mathrm{S}}\right]$, where $\eta$ is the parameter from Theorem 5.1 and $\ell(Q)$ and $\mathrm{t}_{\mathrm{S}}$ are from (5.2).

At this point, we note that the operator D satisfies the following off-diagonal estimates.

Lemma 5.9. Let $U_{t}$ be one of $\mathrm{R}_{t}=(\mathrm{I}+\mathrm{i} t \mathrm{D})^{-1}, \mathrm{P}_{t}=\left(\mathrm{I}+t^{2} \mathrm{D}^{2}\right)^{-1}, \mathrm{Q}_{t}=t \mathrm{D}(\mathrm{I}+$ $\left.t^{2} \mathrm{D}^{2}\right)^{-1}, t \nabla \mathrm{P}_{t}, \tilde{\mathrm{P}}_{t} t$ div, and $\tilde{\mathrm{Q}}_{t}$. Then, there exists $C_{U}>0$ such that, for each $M>0$, there exists a constant $C_{\Delta}>0$ so that

$$
\left\|\chi_{E} U_{t}\left(\chi_{F} u\right)\right\| \lesssim C_{\Delta}\left\langle\frac{\rho(E, F)}{t}\right\rangle^{-M} \exp \left(-C_{U} \frac{\rho(E, F)}{t}\right)\left\|\chi_{F} u\right\|
$$

for every Borel set $E, F \subset \mathcal{M}$ and $u \in \mathrm{L}^{2}(\mathcal{V})$.

This "exponential" version of off-diagonal estimates first appeared as Lemma 5.3 in [10] by Carbonaro, Morris and McIntosh. The proof here is similar, and relies on the commutator estimate (2.3).

With the aid of these tools, we estimate the cancellation term in (5.11). We note that the proof is similar to the corresponding result found in [4], with the exception being the complication arising from the operator $S$ in the following statement. Thus, we give sufficiently detailed recollection of the proof.

Proposition 5.10. Let $S=\mathrm{I}$ or $S=\nabla(\mathrm{iI}+\mathrm{D})^{-1}$. Then,

$$
\int_{0}^{t_{H}(\mathbf{Q})}\left\|\gamma_{t} \mathbb{E}_{t} S\left(\mathrm{I}-\mathrm{P}_{t}\right) u\right\|^{2} \frac{d t}{t} \lesssim\|u\|^{2} .
$$

Proof. First we note that $\mathbb{E}_{t}^{2}=\mathbb{E}_{t}$, and therefore,

$$
\left\|\gamma_{t} \mathbb{E}_{t} S\left(\mathrm{I}-\mathrm{P}_{t}\right) u\right\|=\left\|\gamma_{t} \mathbb{E}_{t} \mathbb{E}_{t} S\left(\mathrm{I}-\mathrm{P}_{t}\right) u\right\| \leq\|A\|_{\infty}\left\|\mathbb{E}_{t} S\left(\mathrm{I}-\mathrm{P}_{t}\right) u\right\| .
$$

By Schur estimate techniques (see Proposition 5.7 in [4]), it suffices to prove that

$$
\left\|\mathbb{E}_{t} S\left(\mathrm{I}-\mathrm{P}_{t}\right) \mathrm{Q}_{s}\right\| \lesssim \min \left\{\left(\frac{s}{t}\right)^{\alpha},\left(\frac{t}{s}\right)^{\alpha}\right\}
$$

for some $\alpha>0$.

Note the identities

$$
\left(\mathrm{I}-\mathrm{P}_{t}\right) \mathrm{Q}_{s}=\frac{t}{s} \mathrm{Q}_{t}\left(\mathrm{I}-\mathrm{P}_{s}\right) \text { and } \mathrm{P}_{t} \mathrm{Q}_{s}=\frac{s}{t} \mathrm{Q}_{t} \mathrm{P}_{s} .
$$


For $t \leq s$, it immediately follows from (5.19) that

$$
\left\|\mathbb{E}_{t} S\left(\mathrm{I}-\mathrm{P}_{t}\right) \mathrm{Q}_{s}\right\| \lesssim\left\|\left(\mathrm{I}-\mathrm{P}_{t}\right) \mathrm{Q}_{s}\right\| \lesssim \frac{t}{s}
$$

For $t>s$, we write

$$
\left\|\mathbb{E}_{t} S\left(\mathrm{I}-\mathrm{P}_{t}\right) \mathrm{Q}_{s}\right\| \lesssim\left\|\mathbb{E}_{t} S \mathrm{Q}_{s}\right\|+\left\|\mathrm{P}_{t} \mathrm{Q}_{s}\right\| \lesssim\left\|\mathbb{E}_{t} S \mathrm{Q}_{s}\right\|+\frac{s}{t},
$$

where the last inequality follows from (5.19). Thus, we only need to prove that there is an $\alpha>0$ such that

$$
\left\|\mathbb{E}_{t} S Q_{s}\right\| \lesssim\left(\frac{s}{t}\right)^{\alpha}
$$

Fix $u \in \mathrm{L}^{2}(\mathcal{V})$ and note that

$$
\left\|\mathbb{E}_{t} S Q_{s} u\right\|^{2}=\sum_{Q \in \mathscr{Q}_{t}}\left\|\mathbb{E}_{t} S \mathrm{Q}_{s} u\right\|_{\mathrm{L}^{2}(Q)}^{2}
$$

If $S=\nabla(\mathrm{iI}+\mathrm{D})^{-1}$, we have that

$$
S \mathrm{Q}_{s}=S s \mathrm{DP}_{s}=\nabla(\mathrm{iI}+\mathrm{D})^{-1} s \mathrm{DP}_{s}=s \nabla \mathrm{P}_{s}-\mathrm{i} s \nabla(\mathrm{iI}+\mathrm{D})^{-1} \mathrm{P}_{s} .
$$

Also, for $x \in Q$,

$$
\mathbb{E}_{t} S \mathrm{Q}_{s} u(x)=f_{Q} s \nabla \mathrm{P}_{s} u d \mu-f_{Q} \mathrm{i} s \nabla \mathrm{P}_{s}(\mathrm{iI}+\mathrm{D})^{-1} \mathrm{P}_{s} u d \mu,
$$

and therefore,

$$
\begin{aligned}
\left\|\mathbb{E}_{t} S \mathrm{Q}_{s} u\right\|_{\mathrm{L}^{2}(Q)}^{2} & =\int_{Q}\left|f_{Q} s \nabla \mathrm{P}_{s} u d \mu-f_{Q} \mathrm{i} s \nabla \mathrm{P}_{s}(\mathrm{iI}+\mathrm{D})^{-1} u d \mu\right|^{2} d \mu \\
& \lesssim \mu(Q)\left|f_{Q} s \nabla \mathrm{P}_{s} u d \mu\right|^{2}+\mu(Q)\left|f_{Q} s \nabla \mathrm{P}_{s}(\mathrm{iI}+\mathrm{D})^{-1} u d \mu\right|^{2} .
\end{aligned}
$$

In the case $S=\mathrm{I}$, we obtain that $\mathbb{E}_{t} S \mathrm{Q}_{s} u=f_{Q} s \mathrm{DP}_{s} u d \mu$, so that

$$
\left\|\mathbb{E}_{t} S Q_{s} u\right\|_{\mathrm{L}^{2}(Q)} \simeq \mu(Q)\left|f_{Q} s \mathrm{DP}_{s} u d \mu\right|^{2}
$$

This latter estimate can be handled if we can handle the former estimate and so it suffices to only consider this case. On noting that $t \simeq \ell(Q)$ from (5.2), by Lemma 5.8

$$
\begin{aligned}
\left|f_{Q} s \nabla \mathrm{P}_{s} u d \mu\right|^{2} \lesssim\left(\frac{s}{t}\right)^{\eta} \frac{1}{\mu(Q)}\left\|\mathrm{P}_{s} u\right\|_{\mathrm{L}^{2}(Q)}^{\eta}\left\|s \nabla \mathrm{P}_{s} u\right\|_{\mathrm{L}^{2}(Q)}^{2-\eta} & \\
& +t^{2}\left(\frac{s}{t}\right)^{2} \frac{1}{\mu(Q)}\left\|\mathrm{P}_{s} u\right\|_{\mathrm{L}^{2}(Q)}^{2}
\end{aligned}
$$


Then, by choosing $p=2 / \eta$ and $q=2 /(2-\eta)$, and by Hölder's inequality and the uniform boundedness of $\mathrm{P}_{s}, s \mathrm{P}_{s}$, and $\mathrm{Q}_{s}=s \mathrm{DP}_{s}$ on $s \in(0,1]$,

$$
\begin{aligned}
\sum_{Q \in \mathscr{Q}_{t}}\left\|\mathrm{P}_{s} u\right\|_{\mathrm{L}^{2}(Q)}^{\eta}\left\|s \nabla \mathrm{P}_{s} u\right\|_{\mathrm{L}^{2}(Q)}^{2-\eta} \\
\quad \lesssim\left(\sum_{Q \in \mathscr{Q}_{t}}\left\|\mathrm{P}_{s} u\right\|_{\mathrm{L}^{2}(Q)}^{2}\right)^{\frac{\eta}{2}}\left(\sum_{Q \in \mathscr{Q}_{t}}\left\|s \nabla \mathrm{P}_{s} u\right\|_{\mathrm{L}^{2}(Q)}^{2}\right)^{\frac{2-\eta}{2}} \\
\lesssim\left\|\mathrm{P}_{s} u\right\|^{\eta}\left(\left\|s \mathrm{DP}_{s} u\right\|^{2}+\left\|s \mathrm{P}_{s} u\right\|^{2}\right)^{\frac{2-\eta}{2}} \lesssim\|u\|^{2}
\end{aligned}
$$

Thus, for $u$ replaced by $(\mathrm{iI}+\mathrm{D})^{-1} u$, we obtain,

$$
\begin{aligned}
\left\|\mathbb{E}_{t} S \mathrm{Q}_{s} u\right\|^{2} & \lesssim\left(\frac{s}{t}\right)^{2}\|u\|^{2}+\left(\frac{s}{t}\right)^{\eta}\|u\|^{2}+\left(\frac{s}{t}\right)^{\eta}\left\|(\mathrm{iI}+\mathrm{D})^{-1} u\right\|^{2} \\
& \lesssim\left(\frac{s}{t}\right)^{2}\|u\|^{2}+\left(\frac{s}{t}\right)^{\eta}\|u\|^{2} .
\end{aligned}
$$

This finishes the proof.

5.5. The Carleson term. We are now left with the task of estimating the last term, the Carleson term in (5.11). Recall that $\nu$ is a local Carleson measure on $\mathcal{M} \times\left(0, t^{\prime}\right]$ (for some $t^{\prime} \in\left(0, \mathrm{t}_{\mathrm{S}}\right]$, where $\mathrm{t}_{\mathrm{S}}$ is the scale we define in $\left.\S 5.1\right)$ if

$$
\|\nu\|_{\mathcal{C}}=\sup _{t \in\left(0, t^{\prime}\right]} \sup _{Q \in \mathscr{Q}_{t}} \frac{\nu(\mathrm{R}(Q))}{\mu(Q)}<\infty
$$

where $\mathrm{R}(Q)=Q \times(0, \ell(Q))$, the Carleson box over $Q$. The norm $\|\nu\|_{\mathcal{C}}$ is the local Carleson norm of $\nu$.

If $\nu$ is a local Carleson measure, then by Carleson's inequality,

$$
\iint_{\mathcal{M} \times\left(0, t^{\prime}\right]}\left|\mathbb{E}_{t}(x) u(x)\right|^{2} d \nu(x, t) \lesssim\|\nu\|_{\mathcal{C}}\|u\|^{2}
$$

for all $u \in \mathrm{L}^{2}(\mathcal{V})$. This is proved for functions in Theorem 4.2 in [23] but we note that the proof carries over mutatis mutandis to our setting.

Since $S$ is a bounded operator, we can reduce Carleson's inequality

$$
\int_{0}^{1}\left\|\gamma_{t} \mathbb{E}_{t} S u\right\|^{2} \frac{d t}{t} \lesssim\|A\|_{\infty}^{2}\|u\|^{2}
$$

to showing that

$$
d \nu(x, t)=\left|\gamma_{t}(x)\right|^{2} \frac{d \mu(x) d t}{t}
$$

is a local Carleson measure with Carleson norm controlled by $\|A\|_{\infty}^{2}$.

Fix a cube $Q \in \mathscr{Q}_{t}$, let $\mathrm{B}_{Q}=\mathrm{B}\left(x_{Q}, C_{1} \ell(Q)\right)$, Note that since we consider $t^{\prime} \leq \mathrm{t}_{\mathrm{S}}$, we have that $3 \mathrm{~B}_{Q} \subset \mathrm{B}\left(x_{\widehat{Q}}, C_{1} \ell(\widehat{Q})\right)$, where $\rho$ is the GBG radius. This is one reason why we fix $\mathrm{t}_{\mathrm{S}} \leq \rho / 5$ in our analysis. 
For $w \in \mathbb{C}^{N}$, let $w^{c}$ denote the local constant extension of $w$ as defined in (5.8), and define $w^{Q}=\chi_{2 B_{Q}} w^{c}$. Then, we note that

$$
\iint_{\mathrm{R}(Q)}\left|\gamma_{t}(x)\right|^{2} \frac{d \mu(x) d t}{t} \lesssim \sup _{|w|_{\mathbb{C}^{N}}=1} \int_{0}^{\ell(Q)} \int_{Q}\left|\gamma_{t} \mathbb{E}_{t} w^{Q}\right|^{2} \frac{d \mu d t}{t},
$$

and therefore, it suffices to prove that

$$
\int_{0}^{\ell(Q)} \int_{Q}\left|\gamma_{t} \mathbb{E}_{t} w^{Q}\right|^{2} \frac{d \mu d t}{t} \lesssim\|A\|_{\infty}^{2} \mu(Q)
$$

for each $|w|_{\mathbb{C}^{N}}=1$.

In order to do this, we split up this integral in the following way:

$$
\begin{aligned}
& \int_{0}^{\ell(Q)} \int_{Q}\left|\gamma_{t} \mathbb{E}_{t} w^{Q}\right|^{2} \frac{d \mu d t}{t} \lesssim \\
& \int_{0}^{\ell(Q)} \int_{Q}\left|\left(\gamma_{t} \mathbb{E}_{t}-\mathbf{Q}_{t}\right) w^{Q}\right|^{2} \frac{d \mu d t}{t}+\int_{0}^{\ell(Q)} \int_{Q}\left|\mathbf{Q}_{t} w^{Q}\right|^{2} \frac{d \mu d t}{t}
\end{aligned}
$$

Proposition 5.11. Let $\mathrm{Q}_{t}: \mathrm{L}^{2}(\mathcal{W}) \rightarrow \mathrm{L}^{2}(\mathcal{V})$ be a family of operators uniformly bounded in $t \in(0,1]$ satisfying (5.4). Then for each cube $Q \in \mathscr{Q}_{t}$,

$$
\int_{0}^{\ell(Q)} \int_{Q}\left|\left(\gamma_{t} \mathbb{E}_{t}-\mathbf{Q}_{t}\right) w^{Q}\right|^{2} \frac{d \mu d t}{t} \lesssim\|A\|_{\infty}^{2} \mu(Q),
$$

whenever $t \in\left(0, t_{3}(\mathbf{Q})\right]$, where $t_{3}(\mathbf{Q})=\min \left\{t_{\mathrm{H}}(\mathbf{Q}), \frac{C_{\mathbf{Q}}}{3 c_{E}}\right\}$. The implicit constant depends on $\mathrm{C}(\mathcal{M}, \mathcal{V}, \mathrm{D}, \tilde{\mathrm{D}})$ and $C_{\Delta, \kappa+1}$ from (5.4).

Proof. First, we note that for $x \in Q, \mathbb{E}_{t} w^{Q}(x)=w^{c}(x)$ and hence, $\gamma_{t}(x) \mathbb{E}_{t} w^{Q}(x)=$ $\left(\mathbf{Q}_{t} w^{c}\right)(x)$. Setting $v=w^{Q}-w^{c}$, we have $\left|\left(\gamma_{t} \mathbb{E}_{t}-\mathbf{Q}_{t}\right) w^{Q}\right|=\left|\mathbf{Q}_{t} v\right|$ almost-everywhere in $Q$.

Letting $C_{j}(Q)=2^{j+1} \mathrm{~B}_{Q} \backslash 2^{j} \mathrm{~B}_{Q}$, and fixing $M>0$ to be chosen later, we estimate via (5.4) and by using Cauchy-Schwartz as in (5.14)

$$
\begin{aligned}
\int_{Q}\left|\mathbf{Q}_{t} v\right|^{2} d \mu & =\int_{Q}\left|\mathbf{Q}_{t}\left(\sum_{j=0}^{\infty} \chi_{C_{j}(Q)}\right) v\right|^{2} d \mu \\
& \lesssim\|A\|_{\infty}^{2} \sum_{j=0}^{\infty}\left\langle\frac{\rho\left(Q, C_{j}(Q)\right)}{t}\right\rangle^{-M} \\
& \quad \exp \left(-2 C_{\mathbf{Q}} \frac{\rho\left(Q, C_{j}(Q)\right)}{t}\right) \int_{\mathcal{M}}\left|\chi_{C_{j}(Q)} v\right|^{2} d \mu
\end{aligned}
$$

First, note that $v(x)=w^{Q}(x)-w^{c}(x)=\chi_{2 \mathrm{~B}_{Q}}(x) w_{i} e^{i}(x)-w_{i} e^{i}(x)$ and hence, $|v(x)| \leq 1$ for almost-every $x$, and thus

$$
\int_{\mathcal{M}}\left|\chi_{C_{j}(Q)} v\right|^{2} d \mu \leq \mu\left(C_{j}(Q)\right) \leq \mu\left(2^{j+1} \mathrm{~B}_{Q}\right) .
$$


Moreover, from ( $\left.\mathrm{E}_{\mathrm{loc}}\right)$ and since $\delta^{j+1}<t \leq \ell(Q)=\delta^{j}$,

$$
\mu\left(2^{j+1} \mathrm{~B}_{Q}\right) \leq \mu\left(\mathrm{B}\left(x_{Q}, 2^{j+1} t C_{1} / \delta\right)\right) \lesssim 2^{\kappa(j+1)} \exp \left(c_{E} \frac{C_{1}}{\delta} 2^{j+1} t\right) \mu(Q) .
$$

Thus, on combining these two inequalities with (5.13) we obtain from (5.24) that

$$
\int_{Q}\left|\mathbf{Q}_{t} v\right|^{2} d \mu \lesssim\|A\|_{\infty}^{2} \frac{t}{\ell(Q)} \mu(Q) \sum_{j=0}^{\infty} 2^{(\kappa-M)(j+1)} \exp \left(\left(\frac{c_{E} C_{1}}{\delta} t-\frac{C_{\mathbf{Q}} C_{1}}{2 \delta}\right) 2^{j+1}\right) .
$$

Thus, by choosing $M>\kappa$, or explicitly, setting $M=\kappa+1$ and choosing $t \leq \frac{C_{\mathbf{Q}}}{3 c_{E}}$, the right hand sum converges. That is,

$$
\int_{Q}\left|\left(\gamma_{t} \mathbb{E}_{t}-\mathbf{Q}_{t}\right) w^{Q}\right|^{2} \frac{d \mu d t}{t} \lesssim\|A\|_{\infty}^{2} \mu(Q)
$$

which completes the proof.

From this, we obtain the following.

Proposition 5.12. Let $\mathrm{Q}_{t}: \mathrm{L}^{2}(\mathcal{W}) \rightarrow \mathrm{L}^{2}(\mathcal{V})$ be a family of operators uniformly bounded in $t \in(0,1]$ satisfying (5.5) and (5.4). Then, whenever $S \in \mathcal{L}\left(\mathrm{L}^{2}(\mathcal{V})\right)$, for every $u \in \mathrm{L}^{2}(\mathcal{V})$, we obtain that

$$
\int_{0}^{t_{3}(\mathbf{Q})}\left\|\gamma_{t} \mathbb{E}_{t} S u\right\|^{2} \frac{d t}{t} \lesssim\|A\|_{\infty}^{2}\|u\|^{2},
$$

where $t_{3}(\mathbf{Q})=\min \left\{t_{\mathrm{H}}(\mathbf{Q}), \frac{C_{\mathbf{Q}}}{3 c_{E}}\right\}$ and where the implicit constants depend on the bound on $\|S\|_{\mathrm{L}^{2} \rightarrow \mathrm{L}^{2}}, C(\mathbf{Q})^{\prime}$ from (5.5), $C_{\Delta, \kappa+1}$ from (5.4), and $\mathrm{C}(\mathcal{M}, \mathcal{V}, \mathrm{D}, \tilde{\mathrm{D}})$.

Proof. This follows from Proposition 5.11 and the computation:

$$
\int_{0}^{\ell(Q)} \int_{Q}\left|\mathbf{Q}_{t} w^{Q}\right|^{2} \frac{d \mu d t}{t} \lesssim \int_{0}^{1}\left\|\mathbf{Q}_{t} w^{Q}\right\|^{2} \frac{d t}{t} \lesssim\|A\|_{\infty}^{2}\left\|w^{Q}\right\|^{2} \lesssim\|A\|_{\infty}^{2} \mu(Q)
$$

where the second inequality comes from the (5.5) assumption on $\mathbf{Q}_{t}$ and the third inequality follows from the fact that spt $w^{Q} \subset 2 \mathrm{~B}_{Q}$ and $\mu\left(2 \mathrm{~B}_{Q}\right) \lesssim \mu(Q)$ by $\left(\mathrm{E}_{\mathrm{loc}}\right)$.

5.6. Proof of the main theorem. Finally, we gather the estimates in $\S 4$ and $\S 5$ to obtain a proof of the main theorem.

Proof of Theorem 2.4. First, we note that, by Proposition 4.6, it suffices to show that

$$
\begin{aligned}
& \int_{0}^{1}\left\|t \tilde{\mathrm{P}}_{t} \operatorname{div} A_{2} \mathrm{P}_{t} f\right\|^{2} \frac{d t}{t} \lesssim\|A\|_{\infty}^{2}\|f\|^{2}, \quad \text { and } \\
& \int_{0}^{1}\left\|\tilde{\mathrm{Q}}_{t} A_{1} \nabla(\mathrm{iI}+\mathrm{D})^{-1} \mathrm{P}_{t} f\right\|^{2} \frac{d t}{t} \lesssim\|A\|_{\infty}^{2}\|f\|^{2}
\end{aligned}
$$


For the first inequality, we set $\mathrm{Q}_{t}=t \tilde{\mathrm{P}}_{t} \operatorname{div} A_{2}$, and noting the identity $t \tilde{\mathrm{P}}_{t} \operatorname{div}=$ $\left(\tilde{\mathrm{Q}}_{t}+\mathrm{i} t \tilde{\mathrm{P}}_{t}\right)\left(\nabla(\mathrm{iI}-\tilde{\mathrm{D}})^{-1}\right)^{*}$, the quadratic estimates for $\tilde{\mathrm{Q}}_{t}$, the boundedness of $\tilde{\mathrm{P}}_{t}$ uniformly in $t$ and the the boundedness of $\nabla(\mathrm{iI}-\tilde{\mathrm{D}})^{-1}$, we obtain

$$
\int_{0}^{1}\left\|\mathrm{Q}_{t} f\right\|^{2} \frac{d t}{t}=\int_{0}^{1}\left\|\left(t \tilde{\mathrm{P}}_{t} \operatorname{div}\right) A_{2} f\right\|^{2} \frac{d t}{t} \lesssim\left\|A_{2} f\right\|^{2} \leq\|A\|_{\infty}^{2}\|f\|^{2} .
$$

Moreover, from Lemma 5.9 with $\mathrm{D}^{\prime}=\operatorname{div}$ and $u=A_{2} f$, we obtain that $\mathbf{Q}_{t}$ satisfies (5.4). Letting $S=$ I Propositions 5.4, 5.10 and 5.12 yields

$$
\int_{0}^{t_{1}(\mathbf{Q})}\left\|t \tilde{\mathrm{P}}_{t} \operatorname{div} A_{2} \mathrm{P}_{t} f\right\|^{2} \frac{d t}{t} \lesssim\|A\|_{\infty}^{2}\|f\|^{2}
$$

for all $f \in \mathrm{L}^{2}(\mathcal{V})$, where $t_{1}(\mathbf{Q})=\min \left\{\mathrm{t}_{\mathrm{H}}(\mathbf{Q}), C_{\mathbf{Q}} /\left(11 c_{E}\right)\right\}$ (from Proposition 5.4), and since $t_{1}(\mathbf{Q}) \leq t_{3}(\mathbf{Q})$ where $t_{3}(\mathbf{Q})$ is defined in Proposition 5.12. We obtain

$$
\int_{t_{1}(\mathbf{Q})}^{1}\left\|t \tilde{\mathrm{P}}_{t} \operatorname{div} A_{2} \mathrm{P}_{t} f\right\|^{2} \frac{d t}{t} \lesssim\|A\|_{\infty}^{2}\|f\|^{2}
$$

from recalling that $\left\|t \tilde{\mathrm{P}}_{t} \operatorname{div} A_{2} \mathrm{P}_{t} f\right\| \lesssim\left\|A_{2}\right\|_{\infty}\|f\|$ uniformly in $t$.

Now, set $\mathrm{Q}_{t}=\tilde{\mathrm{Q}}_{t} A_{1}$ and $S=\nabla(\mathrm{iI}+\mathrm{D})^{-1}$. This $\mathrm{Q}_{t}$ clearly satisfies (5.5) and by Lemma 5.9 it satisfies (5.4). Thus, we are able to apply Propositions 5.10 and 5.12 , but in order to apply Proposition 5.4, it remains to verify that the operator $S$ satisfies $\|\nabla S u\| \lesssim\|\nabla u\|+\|u\|$ whenever $u \in \mathrm{W}^{1,2}(\mathcal{V})$, To this end, we use the assumptions (A8) and (A9) to estimate

$$
\begin{aligned}
\|\nabla S u\| & =\left\|\nabla \nabla(\mathrm{iI}+\mathrm{D})^{-1} u\right\|=\left\|\nabla^{2}(\mathrm{iI}+\mathrm{D})^{-1} u\right\| \\
& \lesssim\left\|\mathrm{D}^{2}(\mathrm{iI}+\mathrm{D})^{-1} u\right\|+\left\|(\mathrm{iI}+\mathrm{D})^{-1} u\right\| \\
& \lesssim\left\|\mathrm{D}\left(\mathrm{iI}+\mathrm{D}^{-1}\right) \mathrm{D} u\right\|+\|u\| \lesssim\|\mathrm{D} u\|+\|u\| \lesssim\|\nabla u\|+\|u\| .
\end{aligned}
$$

We obtain

$$
\int_{0}^{t_{1}(\mathbf{Q})}\left\|\tilde{\mathrm{Q}}_{t} A_{1} \nabla(\mathrm{iI}+\mathrm{D})^{-1} \mathrm{P}_{t} f\right\| \frac{d t}{t} \lesssim\|A\|_{\infty}^{2}\|f\|^{2}
$$

for $f \in \mathrm{L}^{2}(\mathcal{V})$. Similar to our previous calculation,

$$
\int_{t_{1}(\mathbf{Q})}^{1}\left\|\tilde{\mathrm{Q}}_{t} A_{1} \nabla(\mathrm{iI}+\mathrm{D})^{-1} \mathrm{P}_{t} f\right\| \frac{d t}{t} \lesssim\|A\|_{\infty}^{2}\|f\|^{2}
$$

follows from $\left\|\tilde{\mathrm{Q}}_{t} A_{1} \nabla(\mathrm{iI}+\mathrm{D})^{-1} \mathrm{P}_{t} f\right\| \lesssim\left\|A_{1}\right\|_{\infty}\|f\|$ uniformly in $t$.

For the two choices of $\mathbf{Q}_{t}$ which we made, namely $\mathbf{Q}_{t}=t \tilde{\mathrm{P}}_{t} \operatorname{div} A_{2}$ and $\mathbf{Q}_{t}=\tilde{\mathrm{Q}}_{t} A_{1}$, the constants $C_{\Delta, M}$ from (5.4) and $C_{\mathbf{Q}}^{\prime}$ from (5.5) only depend on $\mathrm{C}(\mathcal{M}, \mathcal{V}, \mathrm{D}, \tilde{\mathrm{D}})$ and the constants $C_{S}$ and $C_{G, W}$ from Proposition 5.4. This completes the proof. 


\section{REFERENCES}

1. David Albrecht, Xuan Duong, and Alan McIntosh, Operator theory and harmonic analysis, Instructional Workshop on Analysis and Geometry, Part III (Canberra, 1995), Proc. Centre Math. Appl. Austral. Nat. Univ., vol. 34, Austral. Nat. Univ., Canberra, 1996, pp. 77-136. MR 1394696 (97e:47001)

2. M. F. Atiyah and I. M. Singer, Index theory for skew-adjoint Fredholm operators, Inst. Hautes Études Sci. Publ. Math. (1969), no. 37, 5-26. MR 0285033

3. Pascal Auscher, Steve Hofmann, Michael Lacey, Alan McIntosh, and Philippe. Tchamitchian, The solution of the Kato square root problem for second order elliptic operators on $\mathbb{R}^{n}$, Ann. of Math. (2) 156 (2002), no. 2, 633-654.

4. Andreas Axelsson, Stephen Keith, and Alan McIntosh, Quadratic estimates and functional calculi of perturbed Dirac operators, Invent. Math. 163 (2006), no. 3, 455-497.

5. Lashi Bandara, Density problems on vector bundles and manifolds, Proc. Amer. Math. Soc. 142 (2014), no. 8, 2683-2695. MR 3209324

6. Lashi Bandara and Alan McIntosh, The Kato Square Root Problem on Vector Bundles with Generalised Bounded Geometry, J. Geom. Anal. 26 (2016), no. 1, 428-462. MR 3441522

7. B. Booss-Bavnbek, Basic functional analysis puzzles of spectral flow, J. Aust. Math. Soc. 90 (2011), no. 2, 145-154. MR 2821774

8. Jean-Pierre Bourguignon and Paul Gauduchon, Spineurs, opérateurs de Dirac et variations de métriques, Comm. Math. Phys. 144 (1992), no. 3, 581-599. MR 1158762 (93h:58164)

9. Ulrich Bunke, Comparison of Dirac operators on manifolds with boundary, Proceedings of the Winter School "Geometry and Physics" (Srní, 1991), no. 30, 1993, pp. 133-141. MR 1246627 (95b:58152)

10. Andrea Carbonaro, Alan McIntosh, and Andrew J. Morris, Local Hardy spaces of differential forms on Riemannian manifolds, J. Geom. Anal. 23 (2013), no. 1, 106-169. MR 3010275

11. Paul R. Chernoff, Essential self-adjointness of powers of generators of hyperbolic equations, J. Functional Analysis 12 (1973), 401-414.

12. Michael Christ, $A T(b)$ theorem with remarks on analytic capacity and the Cauchy integral, Colloq. Math. 60/61 (1990), no. 2, 601-628.

13. R. R. Coifman, A. McIntosh, and Y. Meyer, L'intégrale de Cauchy définit un opérateur borné sur $L^{2}$ pour les courbes lipschitziennes, Ann. of Math. (2) 116 (1982), no. 2, 361-387. MR 672839 (84m:42027)

14. Ronald Coifman, Alan McIntosh, and Yves Meyer, The Hilbert transform on Lipschitz curves, Miniconference on partial differential equations (Canberra, 1981), Proc. Centre Math. Anal. Austral. Nat. Univ., vol. 1, Austral. Nat. Univ., Canberra, 1982, pp. 26-69. MR 758451 (85m:42009)

15. Nicolas Ginoux, The Dirac spectrum, Lecture Notes in Mathematics, vol. 1976, Springer-Verlag, Berlin, 2009. MR 2509837 (2010a:58039)

16. Markus Haase, The functional calculus for sectorial operators, Operator Theory: Advances and Applications, vol. 169, Birkhäuser Verlag, Basel, 2006. MR 2244037 (2007j:47030)

17. Emmanuel Hebey, Nonlinear analysis on manifolds: Sobolev spaces and inequalities, Courant Lecture Notes in Mathematics, vol. 5, New York University Courant Institute of Mathematical Sciences, New York, 1999.

18. Tosio Kato, Perturbation theory for linear operators, second ed., Springer-Verlag, Berlin, 1976, Grundlehren der Mathematischen Wissenschaften, Band 132.

19. H. Blaine Lawson, Jr. and Marie-Louise Michelsohn, Spin geometry, Princeton Mathematical Series, vol. 38, Princeton University Press, Princeton, NJ, 1989. MR 1031992 (91g:53001)

20. Matthias Lesch, The uniqueness of the spectral flow on spaces of unbounded self-adjoint Fredholm operators, Spectral geometry of manifolds with boundary and decomposition of manifolds, Contemp. Math., vol. 366, Amer. Math. Soc., Providence, RI, 2005, pp. 193-224. MR 2114489 (2005m:58049)

21. Alan McIntosh, On the comparability of $A^{1 / 2}$ and $A^{* 1 / 2}$, Proc. Amer. Math. Soc. 32 (1972), 430-434. MR 0290169 (44 \#7354) 
22. Andrew J. Morris, Local quadratic estimates and holomorphic functional calculi, The AMSIANU Workshop on Spectral Theory and Harmonic Analysis, Proc. Centre Math. Appl. Austral. Nat. Univ., vol. 44, Austral. Nat. Univ., Canberra, 2010, pp. 211-231.

23. Andrew J. Morris, The Kato square root problem on submanifolds, J. Lond. Math. Soc. (2) 86 (2012), no. 3, 879-910. MR 3000834

24. Peter Petersen, Riemannian geometry, second ed., Graduate Texts in Mathematics, vol. 171, Springer, New York, 2006. MR 2243772 (2007a:53001)

25. John Phillips, Self-adjoint Fredholm operators and spectral flow, Canad. Math. Bull. 39 (1996), no. 4, 460-467. MR 1426691

Lashi Bandara, Institut für Mathematik, Universität Potsdam, D-14476, Potsdam OT Golm, Germany

$U R L:$ http: //www . math. uni-potsdam.de/ ${ }^{\text {bandara }}$

E-mail address: lashi.bandara@uni-potsdam.de

Alan McIntosh, Mathematical Sciences Institute, Australian National University, Canberra, ACT, 2601, Australia

URL: http://maths.anu.edu.au/〜alan

E-mail address: alan.mcintosh@anu.edu.au

Andreas Rosén, Mathematical Sciences, Chalmers University of Technology and University of Gothenburg, SE-412 96, Gothenburg, Sweden

URL: http://www.math. chalmers.se/ rosenan

E-mail address: andreas.rosen@chalmers.se 